\title{
Kinematic alignment of non-interacting CALIFA galaxies ${ }^{\star}$ Quantifying the impact of bars on stellar and ionised gas velocity field
orientations
}

\author{
J. K. Barrera-Ballesteros ${ }^{1,2}$, J. Falcón-Barroso ${ }^{1,2}$, B. García-Lorenzo ${ }^{1,2}$, G. van de Ven ${ }^{3}$, J. A. L. Aguerri ${ }^{1,2}$, \\ J. Mendez-Abreu ${ }^{1,2,4}$, K. Spekkens ${ }^{5}$, M. Lyubenova ${ }^{3}$, S. F. Sánchez ${ }^{6,7,8}$, B. Husemann ${ }^{9,10}$, D. Mast ${ }^{6,8}$, \\ R. García-Benito ${ }^{6}$, J. Iglesias-Paramo ${ }^{6,8}$, A. del Olmo ${ }^{6}$, I. Márquez ${ }^{6}$, J. Masegosa ${ }^{6}$, C. Kehrig ${ }^{6}$, R. A. Marino ${ }^{11}$, \\ L. Verdes-Montenegro ${ }^{6}$, B. Ziegler ${ }^{12}$, D. H. McIntosh ${ }^{13}$, J. Bland-Hawthorn ${ }^{14}$, \\ C. J. Walcher ${ }^{10}$, and the CALIFA Collaboration \\ (Affiliations can be found after the references)
}

Received 22 January 2014 / Accepted 20 May 2014

\begin{abstract}
We present 80 stellar and ionised gas velocity maps from the Calar Alto Legacy Integral Field Area (CALIFA) survey in order to characterise the kinematic orientation of non-interacting galaxies. The study of galaxies in isolation is a key step towards understanding how fast-external processes, such as major mergers, affect kinematic properties in galaxies. We derived the global and individual (projected approaching and receding sides) kinematic position angles (PAs) for both the stellar and ionised gas line-of-sight velocity distributions. When compared to the photometric PA, we find that morpho-kinematic differences are smaller than 22 degrees in $90 \%$ of the sample for both stellar and nebular components and that internal kinematic misalignments are generally smaller than 16 degrees. We find a tight relation between the global stellar and ionised gas kinematic PA consistent with circular-flow pattern motions in both components ( $90 \%$ of the sample has differences smaller than 16 degrees). This relation also holds, generally in barred galaxies across the bar and galaxy disc scales. Our findings suggest that even in the presence of strong bars, both the stellar and the gaseous components tend to follow the gravitational potential of the disc. As a result, kinematic orientation can be used to assess the degree of external distortions in interacting galaxies.
\end{abstract}

Key words. galaxies: kinematics and dynamics - galaxies: photometry - galaxies: structure - galaxies: evolution

\section{Introduction}

To first order, galaxies are rotationally supported disc systems (for a historical review, see Sofue \& Rubin 2001). The observed line-of-sight velocity distributions are expected to show a regular rotation pattern in the form of the so-called "spider diagram", with the position angle (PA) of the minimum and maximum velocities aligned with the optical major axis. However, several physical processes can disturb this regular behaviour. On the one hand, kinematic perturbations can be induced by external factors, such as ram-pressure stripping (e.g., Kronberger et al. 2008) or galactic mergers (e.g., Naab \& Burkert 2003). On the other hand, kinematic departures from regular rotation can be due to internal instabilities, such as spiral arms or bars (Binney \& Tremaine 2008; Sellwood 2013). To disentangle the perturbations from external processes, it is necessary first to do a detailed study of the possible kinematic perturbations due to internal processes in both stars and gas.

Integral-field spectroscopy (IFS) is a unique tool for studying the kinematic properties across the entire galaxy. Even though 3D spectroscopic surveys are now feasible for a large set of galaxies (e.g., GHASP, GH $\alpha \mathrm{FaS}$, ATLAS ${ }^{3 \mathrm{D}}$, SAMI; Epinat et al. 2008; Hernandez et al. 2008; Cappellari et al. 2011; Croom et al. 2012), these surveys have usually focussed on studying the kinematics of early-type systems (e.g., SAURON, ATLAS $^{3 \mathrm{D}}$; Emsellem et al. 2004; Krajnović et al. 2011) or a

\footnotetext{
* Appendices are available in electronic form at http://www . aanda.org
}

specific wavelength range like $\mathrm{H} \alpha$ range in spiral galaxies in different environments (e.g., GHASP and GH $\alpha \mathrm{FaS}$ Fathi et al. 2009; Epinat et al. 2008).

The CALIFA survey (Sánchez et al. 2012) provides a unique opportunity to study the stellar and ionised gas global kinematics simultaneously in a sample of galaxies that covers a wide range of morphological types, stellar masses, and environments. The spectral and spatial coverage provided by the CALIFA IFS data allow us to study the effect of structures, such as bars, in non-interacting galaxies on the global kinematics. Bars are nearly elliptical structures present in disc galaxies. About $40 \%$ to $50 \%$ of nearby disc galaxies observed in optical wavelengths show bars features (e.g., Marinova et al. 2007; Barazza et al. 2008; Aguerri et al. 2009). This fraction is even higher (about 60-70\%) for near-infrared observations (e.g., Eskridge et al. 2000; Knapen et al. 2000; Menéndez-Delmestre et al. 2007). Hydrodynamical simulations of these systems show that the bar component contributes only between 10 and $20 \%$ of the disc potential (e.g., Scannapieco et al. 2010), and yet it can produce important changes in the gas dynamics. Bars are thought to be very efficient in redistributing angular momentum, energy and mass in both luminous and dark matter components (e.g., Weinberg 1985; Debattista \& Sellwood 1998, 2000; Athanassoula 2003; Martinez-Valpuesta et al. 2006; Sellwood 2006; Sellwood \& Debattista 2006; Villa-Vargas et al. 2009). The effects of this angular momentum redistribution is different in gas and stars due to their different properties (e.g., Thielheim \& Wolff 1982). For example, flows are observed within the co-rotation radius towards 
the galaxy centre only in the gas component (e.g., Rozas et al. 2002).

The aim of this paper is to characterise the stellar and the ionised gas kinematics for a sample of non-interacting galaxies by means of their global orientation. In a companion paper (Barrera-Ballesteros et al., in prep.), we will use the results of this study to quantify how much the kinematic of a sample of interacting galaxies differentiates with respect to the kinematics of isolated ones. In other words, the result of this work will yield a yardstick that describes kinematic distortions due to internal processes with which to compare kinematic disturbances induced by major merger events.

The paper is organised as follows. In Sect. 2, we present the sample as well as the methods for extracting the stellar and ionised gas kinematics. The procedure used to directly derive the kinematic PA from the velocity maps is presented in Sect. 3. Section 4 presents the kinematic characterisation of the noninteracting galaxies: morpho-kinematic and kinematic misalignments are compared against different bar strengths, as well as at different radii of barred galaxies. The impact of bars in the velocity field is discussed in Sect. 5. Our findings and main conclusions are summarized in Sect. 6.

\section{Sample and observations}

\subsection{Sample selection}

The sample presented in this work is drawn from the CALIFA mother sample (Sánchez et al. 2012) observed until November 2013. This mother sample includes 939 galaxies selected from the SDSS DR7 (Abazajian et al. 2009). The selection criteria is such that galaxies included are in the nearby Universe with redshifts $0.005<z<0.03$, and their isophotal diameter in the SDSS $r$-band is $45^{\prime \prime} \lesssim D_{25} \lesssim 80^{\prime \prime}$.

Using the SDSS $r$-band images of the observed galaxies, we selected 80 galaxies without evident signatures of interaction such as tidal tails, bridges, rings, shells or any other morphological distortion caused by a merging. Moreover, we considered galaxies isolated from companions within a physical radius of $250 \mathrm{kpc}$, a systemic velocity difference smaller than $1000 \mathrm{~km} \mathrm{~s}^{-1}$, and a difference in magnitude in the SDSS $r$-band images larger than 2 mag (position and systemic velocities were taken from $\mathrm{NED}^{1}$ ). We used a conservative physical radius for rejecting close companions with respect to pair-survey criteria (e.g., Ellison et al. 2008). The systemic velocity selection criteria is supported by recent cosmological simulations (Moreno et al. 2013). Finally, we also selected those galaxies for which stellar and ionised gas signal-to-noise ratios $(\mathrm{S} / \mathrm{N})$ allow reliable estimation of their kinematic properties (see Sect. 2.2).

It is important to note that these selection criteria do not exclude from our sample galaxies with minor companions or galaxies located in the outskirts of groups. However, we estimated the perturbation caused by tidal forces induced by companions by means of the f-value (Varela et al. 2004). Values grater than -2 are required to produce sizeable effects on a disc galaxy. We were able to measure the f-value in 71 objects. We found that $95 \%$ of these galaxies have f-values lower than -2 with a mean value of -4.0 . This suggests that our sample is a fairly good representation of isolated galaxies. In fact, ten galaxies from our sample are also part of the AMIGA sample (Analysis of the interstellar Medium of Isolated GAlaxies,

\footnotetext{
1 NASA/IPAC Extragalactic database. http://ned.ipac. caltech. edu/
}

Verdes-Montenegro et al. 2005, CALIFA id: 2, 30, 131, 152, $275,743,748,777,779,856)$. AMIGA sample is defined by strict isolation criteria, and it shows different physical properties at all wavelengths studied so far than galaxies in denser environments (even field galaxies). These ten galaxies are located in the region of lower tidal forces and number density of AMIGA full sample (Fig. 6 in Verley et al. 2007).

Our non-interacting sample includes different morphological types, bar strengths, and a relatively wide range of luminosities $\left(-24 \lesssim M_{r} \lesssim-19\right.$; where $M_{r}$ is the absolute magnitude in SDSS $r$-band). Morphological types and bar strengths were obtained by visual inspection of the SDSS $r$-band images by different members of the collaboration (Walcher et al. 2014, see also Table B.1). Even more, the sample is dominated by $\mathrm{Sb}$ and Sc morphological types, which are also the dominant population of the AMIGA sample, hence characteristic of isolated galaxies. Finally, from a visual inspection of the velocity maps we excluded the early-type galaxy NGC 5623 from our sample. This galaxy presents a stellar counter-rotating disc, presumably originated from an early encounter, and it will be thus included in the sample of interacting galaxies of Barrera-Ballesteros et al. (in prep.).

\subsection{CALIFA velocity maps}

Observations were carried out using the PPAK instrument at Calar Alto Observatory (Roth et al. 2005). Its main component consists of 331 fibers of 2 ". 7 diameter each, concentrated in a single hexagon bundle covering a field-of-view of $74^{\prime \prime} \times 64^{\prime \prime}$, with a filling factor of $\sim 60 \%$. Three dithered pointings were taken for each object to reach a filling factor of $100 \%$ across the entire FoV. The final data cube consists of more than 4000 spectra at a sampling of $1^{\prime \prime} \times 1^{\prime \prime}$ per spaxel (details in Husemann et al. 2013). The CALIFA survey has two spectral setups, V500 and V1200 with low and intermediate resolution, respectively. Objects included in this work where observed in the V500 setup. This setup has a nominal resolution of $\lambda / \Delta \lambda \sim 850$ at $\sim 5000 \AA$ and its nominal wavelength range is $3745-7300 \AA$. However, the final data cube has an homogenized spectral resolution (FWHM) over the entire wavelength range of $6.0 \AA$ and the wavelength sampling per spaxel is $2.0 \AA$. The total exposure time per pointing is fixed for all the observed objects to $45 \mathrm{~min}$. The data reduction is performed by a pipeline designed specifically for the CALIFA survey. Detailed reduction process is explained by Sánchez et al. (2012), and improvements on this pipeline are presented by Husemann et al. (2013).

The stellar kinematic extraction method for the CALIFA survey will be presented in Falcón-Barroso et al. (in prep.). Here we highlight the main steps. Spaxels with continuum $S / N<3$ in the original V500 cube were considered unreliable and were therefore not considered in further analysis. To achieve a minimum $\mathrm{S} / \mathrm{N}$ of 20 , we used a Voronoi-binning scheme for optical IFS data implemented by Cappellari \& Copin (2003). We refer to these Voronoi bins as "voxels". From this selection in the continuum $\mathrm{S} / \mathrm{N}$, a large portion of the voxels $(\sim 80 \%)$ have the same size as the spaxels of the cube. The remaining fraction of the voxels are located in the outer regions of the galaxies and include a range between three and five spaxels. To derive the lineof-sight velocity maps, for each cube, a non-linear combination of a subset of stellar templates from the Indo-US library (Valdes et al. 2004) is fit to each voxel using the penalized pixel-fitting method (pPXF, Cappellari \& Emsellem 2004). Errors for each voxel, determined via Monte Carlo simulations, range from 5 

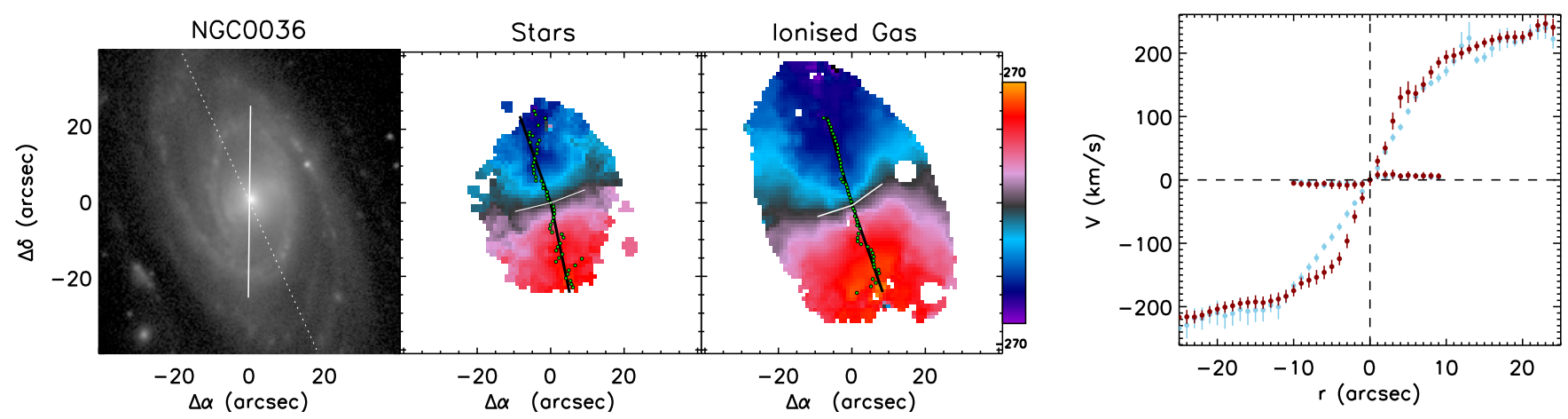

Fig. 1. Example of the method used to determine projected kinematic position angles (PAs) from the velocity maps of the galaxy NGC 36. Leftmost panel shows the SDSS $r$-band image of the galaxy. White solid line represents the photometric PA measured at the same galactocentric distance as the kinematic PA ( $\mathrm{PA}_{\text {morph }}$, measured from the $r$-band image; see Sect. 3). Dashed line represents the photometric PA measured at the outermost isophotes of the image $\left(\mathrm{PA}_{\text {morph }}^{\text {out }}\right)$. Middle-left and middle-right panels show the stellar and ionised gas velocity maps, respectively. Green points highlight the locations where the maximum velocities are located at a given radius, determined from the position-velocity diagram. Black lines for each kinematic side represent the average kinematic $\mathrm{PA}\left(\mathrm{PA}_{\mathrm{kin}}\right)$, while white thin lines along the zero-velocity curve show the average minor kinematic PA. Right panel shows the distance from the galactic centre versus the maximum for the stellar (blue points) and ionised gas (red points) components. The curve along $0 \mathrm{~km} \mathrm{~s}^{-1}$ represents the velocities along the zero-velocity curve. The error bars represent the uncertainty in velocity determined from Monte Carlo simulations.

to $20 \mathrm{~km} \mathrm{~s}^{-1}$ for inner to outer voxels, respectively. These uncertainties are smaller than the spectral sampling per spaxel from the data cubes. Since each spaxel offers a wide range of absorption lines to fit its line-of-sight velocity, the spectral location of these absorption features can be determine even at smaller scales than the spectral sampling. Figure 1 (middle-left panel) shows an example of a stellar kinematic maps for one of our galaxies.

The detailed description of the ionised-gas kinematic extraction method for the CALIFA survey is provided by García-Lorenzo et al. (2014). Briefly, to obtain the ionisedgas emission in each spaxel we subtracted the stellar continuum spectra derived from the best stellar pPXF fit in its corresponding voxel. No binning was done for the ionised gas. We assumed that the stellar populations in each voxel are rather smooth and do not change from spaxel to spaxel within each voxel. A cross-correlation (CC) method is used to measure the velocity of the ionised-gas (see García-Lorenzo 2013, for details on the method). The method compares the spectrum in each spaxel in a given wavelength range with a template that includes the $\mathrm{H} \alpha+[\mathrm{NII}] \lambda \lambda 6548,6584$ emission lines $(6508-6623 \AA)$. The template corresponds to a Gaussian model for each emission line in the given wavelength range, shifted to the systemic velocity reported in NED, and assumes a velocity dispersion equal to the instrumental resolution $\left(\sigma \sim 90 \mathrm{~km} \mathrm{~s}^{-1}\right.$ at the $\mathrm{H} \alpha$ emission line). We selected spaxels with $S / N>8$ in this emission line for the ionised gas velocity maps. García-Lorenzo et al. (2014) present examples of the kinematic fitting per spaxel and the extraction of the ionised gas component. Figure 1 (middleright panel) shows an example of an ionised-gas velocity maps. Estimated uncertainties in the location of the maximum of the $\mathrm{CC}$ function are $\sim 10 \mathrm{~km} \mathrm{~s}^{-1}$. Both the pPXF and the CC methods are able to determine velocity dispersion maps; however, due to the low spectral resolution of the V500 data, we did not attempt any further analysis of these maps. They are also not required for the work presented here.

\section{Kinematic position angles from velocity maps}

Appendix $\mathrm{C}$ shows the complete set of stellar and ionised gas velocity maps for the galaxies in this study. Some of them are also presented in Falcón-Barroso et al. (in prep., for stellar kinematics in the V1200 setup) and García-Lorenzo et al. (2014, for the ionised gas).

In the past years, several analytic methods have been developed to determine kinematic properties from two-dimensional velocity distributions. For late-type galaxies, these models often consider a symmetric velocity distribution, either by assuming a thin disc geometry (e.g., Barnes \& Sellwood 2003; Epinat et al. 2008) or symmetrical radial distortions with respect to some axis (Spekkens \& Sellwood 2007). An alternative approach is a generalisation of the isophote fitting (i.e. the standard task ellipse of $\mathrm{IRAF}^{2}$ ) to higher-moments of the LOSVD (e.g. velocity), which assumes that along a best fitting ellipse the odd moments (such as velocity) follow a simple cosine law (Krajnović et al. 2006). Since our wish is to measure kinematic position angles separately on both the approaching and the receding sides of the galaxy, we use here a different approach.

The aim of this paper is to establish the observed (i.e., projected) typical ranges of kinematic parameters expected for noninteracting galaxies. From those kinematic values, we will be able to shade some light on the possible kinematic distortions induced by an interaction. For this reason, we provide in this study a quantitative characterisation of the kinematics of galaxies through parameters derived directly from their stellar and ionised gas velocity distributions. No assumption about the behaviour of the galactic components is made. We use the same method on our sample of interacting galaxies in order to make a consistent comparison. A rotational velocity field can be caracterized by several parameters: kinematic centre, systemic velocity, global orientation of the field (i.e., kinematic PA), and its inclination with respect to the sky's plane. We restrict ourselves only to kinematic projected properties of the sample. We consider that an estimation of the inclination via the velocity distribution would require assuming an intrinsic behaviour on the kinematic component (e.g., tilted-ring modelling, Józsa et al. 2007). Although this could be the case of some of the noninteracting galaxies presented in this study (restricted even only to the nebular component), it might not be the case for highly

2 IRAF is distributed by the Optical Astronomy Observatory, which is operated by the Association of Universities for research in Astronomy (AURA) under cooperative agreement with the National Science Fundation. 
asymmetric velocity fields, such as those exhibited by interacting/merging galaxies. In the work presented here, we focus mainly on characterising the velocity fields via their different components kinematic PAs.

To determine the global orientation of the velocity maps, it is first necessary to set the kinematic centre. For an ideal, rotating disc the kinematic centre should be at the position with the largest velocity gradient (or gradient peak, GP hereafter) and it also should be in agreement with the optical nucleus. The reader is referred to García-Lorenzo et al. (2014) for details of the method used to determine the velocity gradient in the CALIFA ionised gas velocity fields. Briefly, in a region of $10^{\prime \prime} \times 10^{\prime \prime}$ centred on the optical nuclei we determine the velocity gradient in each spaxel with respect to the surrounding spaxels. Then, we select the positions where the velocity gradient is larger than its average inside this box. Finally, the GP is estimated from the weighted average location of the selected positions, using the velocity gradient at each location as weights.

For an observed velocity field, the GP does not always coincided with the optical nucleus (ON hereafter). We take the size of the original fiber as the minimum distance to report an offset between the GP and ON (i.e., 2.7"). We find that a large fraction of the sample in their stellar component do not present a significant offset between these two positions (69/80) with a mean offset of 1.5 and standard deviation of 1.1 arcsec. In the rest of the stellar velocity fields, this offset is due to possible $\mathrm{SNe}$ in the central region (e.g., NGC 7478) or the possibility in barred galaxies that the GP is located at the end of bar (e.g., NGC 6004). The ionised gas present similar offsets (mean offset of 1.3 and standard deviation of 0.9 arcsecs). However the number of galaxies with small offsets is larger (74/80). The galaxies with large offsets are either edge-on galaxies (e.g., UGC 841) or patchy in emission (e.g., NGC 4956). Finally, as kinematic centre we chose either ON or GP, depending on the best symmetry of the rotational curve (see details below). In all the cases with large offset in the GP (in both components), the ON yields a more symmetric velocity curve than the GP. This yields that in $21 / 80$ and $5 / 80$ velocity fields, the ON was selected as the kinematic centre for the stellar and ionised gas components, respectively. As we note above, most of the offsets are smaller than the original spatial resolution of the instrument, in particular, for the stellar component where spatial binning is done (although small for the nuclear region). This yields a larger number of fields where the ON was selected as kinematic centre. In Tables B.2 and B.3, we list the gradient peak location with respect to the optical centre when the choice for the kinematic centre was the GP, and the systemic velocity derived for both components as the mean value within a $2.7^{\prime \prime}$ aperture centered on the kinematic centre.

The major (projected) kinematic axis ( $\mathrm{PA}_{\text {kin }}$, hereafter) measures the global kinematic orientation. It can be determined directly from the positions of the spaxels defining the kinematic lines of nodes (Nicholson et al. 1992). In practice, we plot the radial velocity for all the spaxels up to a given radius on a positionvelocity diagram centred in the kinematic centre previously assigned (see right panel of Fig. 1). We then choose those spaxels with the maximum (minimum) projected velocity at the receding (approaching) side at different radii. Finally, for each side, we identify the selected spaxels in the velocity map. The average of their polar coordinates provides an estimation of $\mathrm{PA}_{\mathrm{kin}}$, and their standard deviation $\left(\delta \mathrm{PA}_{\mathrm{kin}}\right)$ measures the scatter of these points around the straight line defining the kinematic PA. Following a similar procedure, we also determine the minor (receding and approaching) kinematic PA by selecting the spaxels with the lowest velocity differences with respect to the kinematic centre. To compare the stellar and the ionised gas kinematic PA, we pick the common maximum radius where both components can be measured. Although $\mathrm{PA}_{\text {kin }}$ is usually defined as the angle between the north and the receding side of the velocity field (e.g., Schoenmakers et al. 1997), for the sake of homogeneity, we report the approaching and receding kinematic PAs independently $\left(\mathrm{PA}_{\text {kin,app }}\right.$ and $\left.\mathrm{PA}_{\text {kin,rec }}\right)$ between 0 and 180 degrees from north to east. Typical errors in $\mathrm{PA}_{\mathrm{kin}}$ and $\delta \mathrm{PA}_{\mathrm{kin}}$ are of the order of $7^{\circ}$ obtained from Monte Carlo simulations. We have also investigated the uncertainties introduced by the Voronoi binning in the stellar velocity maps (by artificially increasing the level of binning in a few of our galaxies) and established that they could bias the determination of $\mathrm{PA}_{\text {kin }}$ at most $4^{\circ}$.

In this study, for comparison, we also estimate the photometric orientations $\mathrm{PA}_{\text {morph }}$ of the galaxies by fitting an ellipse model using the IRAF task ellipse on the isophotes of the SDSS $r$-band image at two galactocentric distances: 1) at the same galactocentric distance where $\mathrm{PA}_{\text {kin }}$ are derived $\left(\mathrm{PA}_{\text {morph }}\right.$, see left panel of Fig. 1) and 2) at the outermost isophotes of the image ( $\left.\mathrm{PA}_{\mathrm{morph}}^{\text {out }}\right)$. We use the ellipticity of the latter $(\epsilon)$ as an homogeneous proxy for the inclination of the galaxies in our sample (see Table B.1). All the position angles used in this work, as well as the radial distances where they have been computed, are presented in Tables B.2 and B.3.

\section{Kinematic alignment of galaxies}

\subsection{Global morpho-kinematic misalignments}

In Fig. 2 we show the morpho-kinematic PA misalignment (i.e., $\mathrm{PA}_{\text {morph }}-\mathrm{PA}_{\mathrm{kin}}$ ) for the stellar and ionised-gas components (top and middle panels, respectively) against the total stellar mass of the galaxies (from Walcher et al. 2014). Since there is no preference between the kinematic approaching and receding $\mathrm{PA}$, both of them were compared with respect to $\mathrm{PA}_{\text {morph }}$. We do not find any trend between the stellar mass and the morphokinematic PA misalignment, in any component, for our sample of galaxies.

About $90 \%$ of the galaxies have morpho-kinematic misalignments smaller than $21^{\circ}$ (stellar) $/ 22^{\circ}$ (ionised gas). The mean misalignment and standard deviation are $\sim 0^{\circ}$ and $\sim 10^{\circ}$, respectively, in both components. This indicates a global agreement between morphology and kinematics regardless the morphological type or bar strength. Barred galaxies present the larger morphokinematic difference and the larger error bars in both components (see tags in Fig. 2). On the one hand, larger differences in the stellar component are observed in IC 1683, NGC 5735, and NGC 171. For the two first galaxies, the kinematic PA is similar to $\mathrm{PA}_{\text {morph }}^{\text {out }}$ rather than $\mathrm{PA}_{\text {morph }}$. However, $\mathrm{PA}_{\text {kin }}$ of NGC 171 is not similar to any of the photometric PA presented here (see Fig. 4). On the other hand, for the ionised component, NGC 3687 and NGC 5735 present dissagrements with the photometric and kinematic PA. Similar to the stellar component, these galaxies seem to be more aligned to $\mathrm{PA}_{\text {morph }}^{\text {out }}$ rather than $\mathrm{PA}_{\text {morph }}$. In Sect. 4.3, we study the impact of deriving the kinematic PA at different radii, in particular for barred galaxies.

Krajnović et al. (2011) find that $90 \%$ of the ATLAS ${ }^{3 \mathrm{D}}$ galaxies display stellar morpho-kinematic misalignments smaller than $16^{\circ}$. From a sample of 24 early-type bulges, Falcón-Barroso et al. (2006) find that $80 \%$ showed stellar morpho-kinematic differences smaller than $20^{\circ}$. Our results, based on a sample of non-interacting galaxies with a wide range of morphological types, are consistent with those findings. For the ionised-gas, 


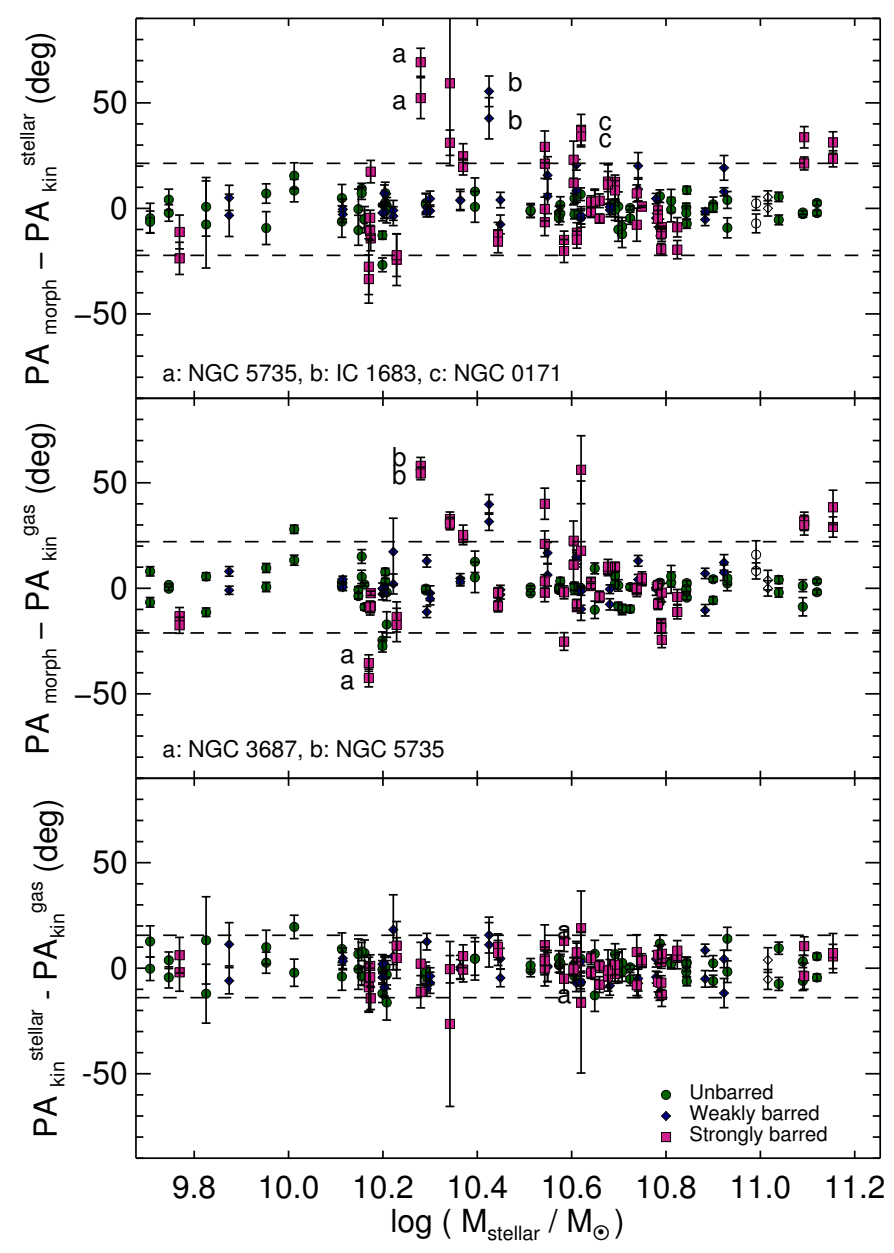

Fig. 2. Difference between the morphological PA $\left(\mathrm{PA}_{\text {morph }}\right)$ and kinematic PA ( $\left.\mathrm{PA}_{\text {kin }}\right)$ against the total stellar mass for the stellar (top) and the ionised gas (middle), as well as the difference between the stellar and ionised-gas kinematic PA (bottom). Both kinematic sides of the velocity maps were compared with $\mathrm{PA}_{\text {morph }}$ (top panels) and between each component (bottom panel). In each panel, the green-filled circles represent unbarred galaxies, blue diamonds are weakly barred galaxies and violet squares correspond to barred galaxies. For early-type galaxies we use open circles. Dashed lines represent the $2 \sigma$ dispersion of the sample. Labels indicate the $3 \sigma$ outliers. Error bars are determined from Monte Carlo simulations.

the Fabry-Perot survey of late-type spirals and irregular galaxies, GHASP (Epinat et al. 2008), reports $85 \%$ of the objects with morpho-kinematic misalignments smaller than $15^{\circ}$. These results also agree with our findings. Similar results have also been found for smaller samples (e.g., Kutdemir et al. 2008).

Although we present projected kinematic properties of noninteracting galaxies, in Appendix A, we study the possible impact of the inclination for determining of kinematic properties. In Fig. A. 2 we plot the previous morpho-kinematic misalignments with respect to the photometric ellipticity as a proxy for the inclination ( $\epsilon$, see Sect. 3). As is expected, larger misalignments are found at low ellipticities, whereas at high ellipticities the morpho-kinematic misalignements get reduced by the projection effects. However, we suggest that even at low ellipticities these misalignments reduced significantly (see Sect. 4.3).

Our results suggest that for a non-interacting galaxy, the misalignment between the photometric and global (stellar- and/or ionised gas-) kinematic orientation is small for a wide range of morphologies and stellar masses (smaller than $20^{\circ}$, with a mean value of $\sim 0^{\circ}$ ). This also held for galaxies with strict isolation critera (i.e., AMIGA subsample), where the average misalignment for these galaxies is $\sim 1^{\circ}$ with a standard deviation of $8^{\circ}$ and $5^{\circ}$ for the stellar and ionised gas component, respectively. As we noted previously, only a small fraction of galaxies (2/80, barred) show large morpho-kinematic misalignments.

In the bottom panel of Fig. 2 we show the difference between the stellar and ionised gas kinematic PA (i.e., $\mathrm{PA}_{\mathrm{kin}}^{\text {stellar }}-\mathrm{PA}_{\mathrm{kin}}^{\mathrm{gas}}$ ). As in previous plots, we separate our sample according to their different bar strengths. In this stellar mass range, we do not find any trend. Barred and unbarred galaxies present similar alignments between their components. In fact, the mean difference for these components orientations is $\sim 1^{\circ}$ with a standard deviation of $\sim 7^{\circ}$. For $90 \%$ of the galaxies, the global orientation of the stellar and the ionised gas velocity fields agrees, with differences smaller than $16^{\circ}$. The only galaxy that seems to present large kinematic misalignment between both components is the late-type barred galaxy NGC 171. The large uncertainties in the ionised gas component, as well as the fact that the locations of maximum velocity present a large deviation from a straight line in the gas component, suggest that the strong bar is the driver that distorts the ionised gas velocity field. From the SDSS $r$-band image, this object presents a bar as large as the FoV of the instrument with an extended ring. This is the only galaxy with these features in the sample presented here. In Fig. A.4 we plot these misalignments with respect to the ellipticity. For a wide range of inclinations, the alignment between both components is consistent. Summarizing, our measurements reveal small morphokinematics misalignments for both component, as well as small stellar versus gas kinematic misalignments for barred galaxies, comparable to those for unbarred galaxies. At the spatial and spectral resolution of CALIFA survey, both components thus seem to follow a similar kinematic pattern even in the presence of bars.

\subsection{Internal kinematic PA misalignments}

In Sect. 3 we estimated kinematic PA from the approaching and receding side and their departures from a straight line $\left(\mathrm{PA}_{\text {kin,rec }}\right.$, $\left.\mathrm{PA}_{\text {kin,app }}, \delta \mathrm{PA}_{\text {kin,rec }}, \delta \mathrm{PA}_{\text {kin,app }}\right)$. This allows us to study the internal kinematic misalignment, defined as $\left|\mathrm{PA}_{\text {kin,rec }}-\mathrm{PA}_{\text {kin,app }}\right|-$ $180^{\circ}$, which should be zero for a regular rotational pattern. In Fig. 3 we present these misalignments for the stellar (left) and the ionised gas (right) components.

We find that $\sim 92 \%$ of the objects have internal kinematic misalignment smaller than $15^{\circ}$ for both the stellar and the ionised gas. This is for different stellar masses and ellipticities (see Fig. A.3). Assuming that our objects are a representative sample of isolated galaxies, we suggest that any distortion in the line-of-sight velocity distribution that produces a larger kinematic misalignment than those values is not caused by internal processes. The kinematic deviations from a straight line $\delta \mathrm{PA}_{\text {kin }}$ range from $5^{\circ}$ to $40^{\circ}$ in both components for a large part of the sample $(\sim 90 \%)$. The mean value of these deviations is twice more for the stellar component than the ionised gas $\left(\sim 15^{\circ}\right.$ and $\sim 7^{\circ}$ ). Their standard deviation is half for gas than the stellar component $\left(\sim 4^{\circ}\right.$ and $8^{\circ}$, respectively). In Fig. 5 (top panel) we plot the distribution of $\delta \mathrm{PA}_{\mathrm{kin}}$ for different barred strengths. That mean deviation of the stellar component is almost twice the deviation from the ionised gas could be explained by different factors. On the one hand, although we are allow to determine the stellar velocity with relatively small uncertainty in each spaxel, 

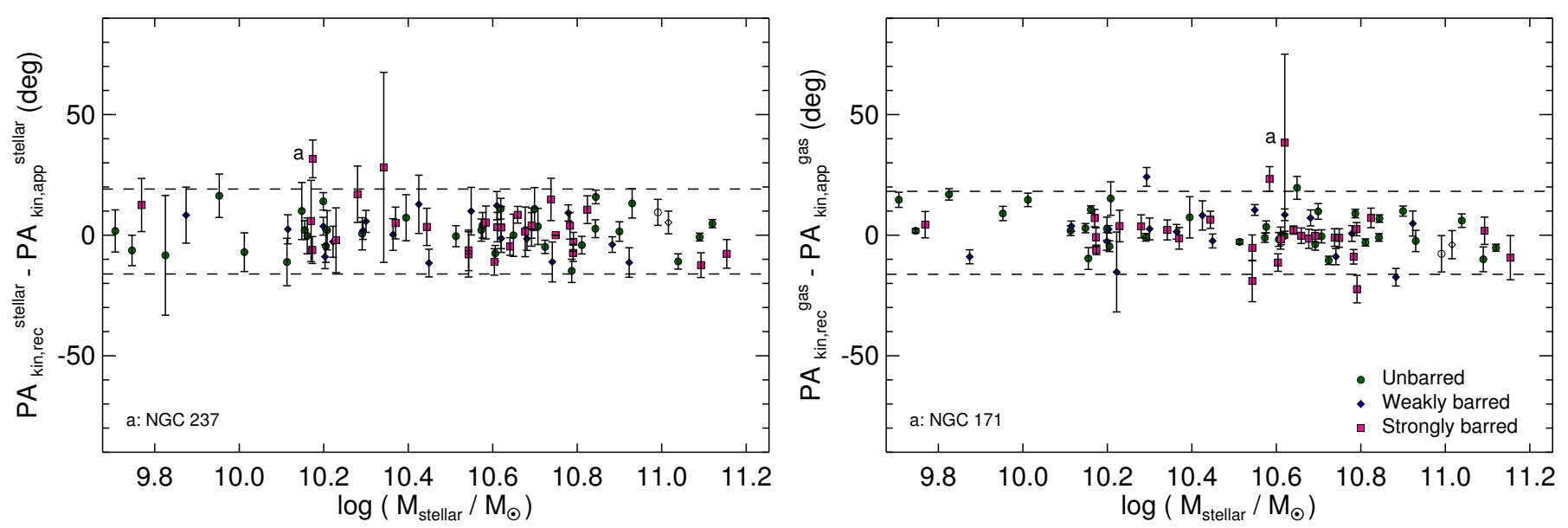

Fig. 3. Internal kinematic misalignment between the receding $\mathrm{PA}\left(\mathrm{PA}_{\mathrm{kin}, \mathrm{ec}}\right)$ and approaching $\left(\mathrm{PA}_{\mathrm{kin}, \mathrm{app}}\right)$ kinematic position angle against the stellar mass for the stellar (left panel) and the ionised gas components (right panel). In each panel the green-filled circles represent the unbarred galaxies, blue diamonds correspond to weakly barred galaxies and violet squares represent the barred galaxies. Open circles represent the early-type galaxies. Dashed lines represent the $2 \sigma$ dispersion of the sample $\left(\sim 15^{\circ}\right)$. Labels indicate the $3 \sigma$ outliers. Error bars are determined from Monte Carlo simulations.

the change in velocity for joint spaxels could be smaller than the spectral resolution leading in a higher scatter in the positions of maximum velocity. On the other hand, the method used here to derive kinematic properties is based entirely on the symmetry that a rotational velocity field should display. Therefore, any deviation translates into a perturbation in any of the parameters we are using to quantify our velocity fields. Small $\delta \mathrm{PA}_{\mathrm{kin}}$ in the ionised gas indicates that gas lies in a thin disc, while the stellar component present "dynamical-heated" structures (e.g., dynamical heated disc, pressure-supported bulge, etc.).

As we mention above, the barred galaxy NGC 171 is the object where it is more difficult to trace a symmetric velocity field in its ionised gas component (see Appendix C), the deviation of the maximum-velocity positions from a straight line is of the order of $\delta \mathrm{PA}_{\mathrm{kin}} \sim 90^{\circ}$. In Sect. 5, we discuss the possible relation of $\delta \mathrm{PA}_{\mathrm{kin}}$ with the level of bar strength of the non-interacting galaxies.

\subsection{Dependence on the measuring radius}

In previous sections, to compare the $\mathrm{PA}_{\mathrm{kin}}$ of both components, we used the same distance to average the polar coordinates of positions defined by the lines of nodes (see Sect. 3). In our data, usually the stellar velocity map limits this distance where the average is done. For barred galaxies, it is similar (and a few times smaller) than half the length of the bar (see $r_{\text {bar }}$ in Table B.1 and $r$ in Tables B.2, and B.3). For the majority of the galaxies, the ionised gas extends further out for these galaxies. Our results could therefore be biased because of measuring the average kinematic orientation on a distance similar or close to the bar length.

To study the impact of this distance on the global orientation of the galaxies, we calculated the morpho-kinematic misalignments at different radii in a sub-sample of 49 low-inclined galaxies $(\epsilon<0.5)$. $\mathrm{PA}_{\mathrm{kin}}$ is computed up to two different radius, $r_{\text {out }}$ and $r_{\text {in }}$ (see Table B.4). The former defines the largest extension of the velocity map in any component. For barred galaxies $r_{\text {in }}$ defines the bar length, for unbarred galaxies we define it as half of the $r_{\text {out }}$. $\mathrm{PA}_{\text {kin }}\left(r_{\text {out }}\right)$ is the average of the polar coordinates from $r_{\text {in }}$ to $r_{\text {out }}$, while $\mathrm{PA}_{\text {kin }}\left(r_{\text {in }}\right)$ is the average from the kinematic centre up to $r_{\text {in }}$.
The left-hand panels of Fig. 4 show, for both components, the morpho-kinematic misalignment with respect to the orientation of an ellipse in the outermost region of each galaxy ( $\mathrm{PA}_{\text {morph }}^{\text {out }}$ ). Red symbols correspond to misalignments with $\mathrm{PA}_{\text {kin }}\left(r_{\text {in }}\right)$, while blue ones represent misalignments for $\mathrm{PA}_{\text {kin }}\left(r_{\text {out }}\right)$. In most of the cases, $\mathrm{PA}_{\text {morph }}^{\text {out }}$ is measured at larger radii than $\mathrm{PA}_{\text {kin }}\left(r_{\text {out }}\right)$. Right panels compare the difference between $\mathrm{PA}_{\text {kin }}\left(r_{\text {in }}\right)$ and $\mathrm{PA}_{\text {kin }}\left(r_{\text {out }}\right)$. Figure 4 illustrates the similar alignment of the kinematic PA for different morphologies at the different measuring radius, $r_{\text {in }}$ and $r_{\text {out }}$, in particular for barred galaxies. On the one hand, the comparison of kinematic PAs and $\mathrm{PA}_{\text {morph }}^{\text {out }}$ (left panels) reveals that $90 \%$ of the objects have differences smaller than $16^{\circ}\left(20^{\circ}\right)$ for the outer (inner) region in the stellar component. Differences became smaller for the ionised gas $\left(13^{\circ}\right.$ and $20^{\circ}$ for outer and inner regions, respectively). On the other hand, when we compare $\mathrm{PA}_{\text {kin }}$ at the inner and outer radii (right panels), we find that $90 \%$ of the galaxies have differences smaller than $20^{\circ}\left(16^{\circ}\right)$ for the stellar (ionised gas) component. These plots quantify what we observed in velocity fields; at different radii the orientation of the major kinematic PA remains rather constant. Moreover, at different radii, $\mathrm{PA}_{\mathrm{kin}}$ seems to be aligned with the orientation of the major photometric axis rather than other morphological local features such as bars. We find one outlier in both components: the strongly barred late-type galaxy (NGC 171). This galaxy presents the lowest ellipticity in the sample (see Fig. A.2), making it difficult to determine a reliable estimation of $\mathrm{PA}_{\mathrm{morph}}^{\text {out }}$. From these morpho-kinematic differences, as well as from the differences presented in Sects. 4.1 and 4.2, we suggest that for this sample of non-interacting galaxies, the stars and the ionised gas follow the potential of the disc.

\section{The impact of bars in velocity maps}

Simultaneous comparisons of the stellar and ionised gas resolved kinematics in barred galaxies are rather scarce. Falcón-Barroso et al. (2006) studied the velocity distributions of 24 spiral bulges. This sample included nine barred galaxies. Kinematic misalignments between the two components for these galaxies ranged from $1^{\circ}$ to $38^{\circ}$. A subsequent detailed study of the galaxy NGC 5448 (Fathi et al. 2005) revealed a kinematic misalignment between these two components of $25^{\circ}$. These differences are explained by the fact that the morphological PA of the galaxies 

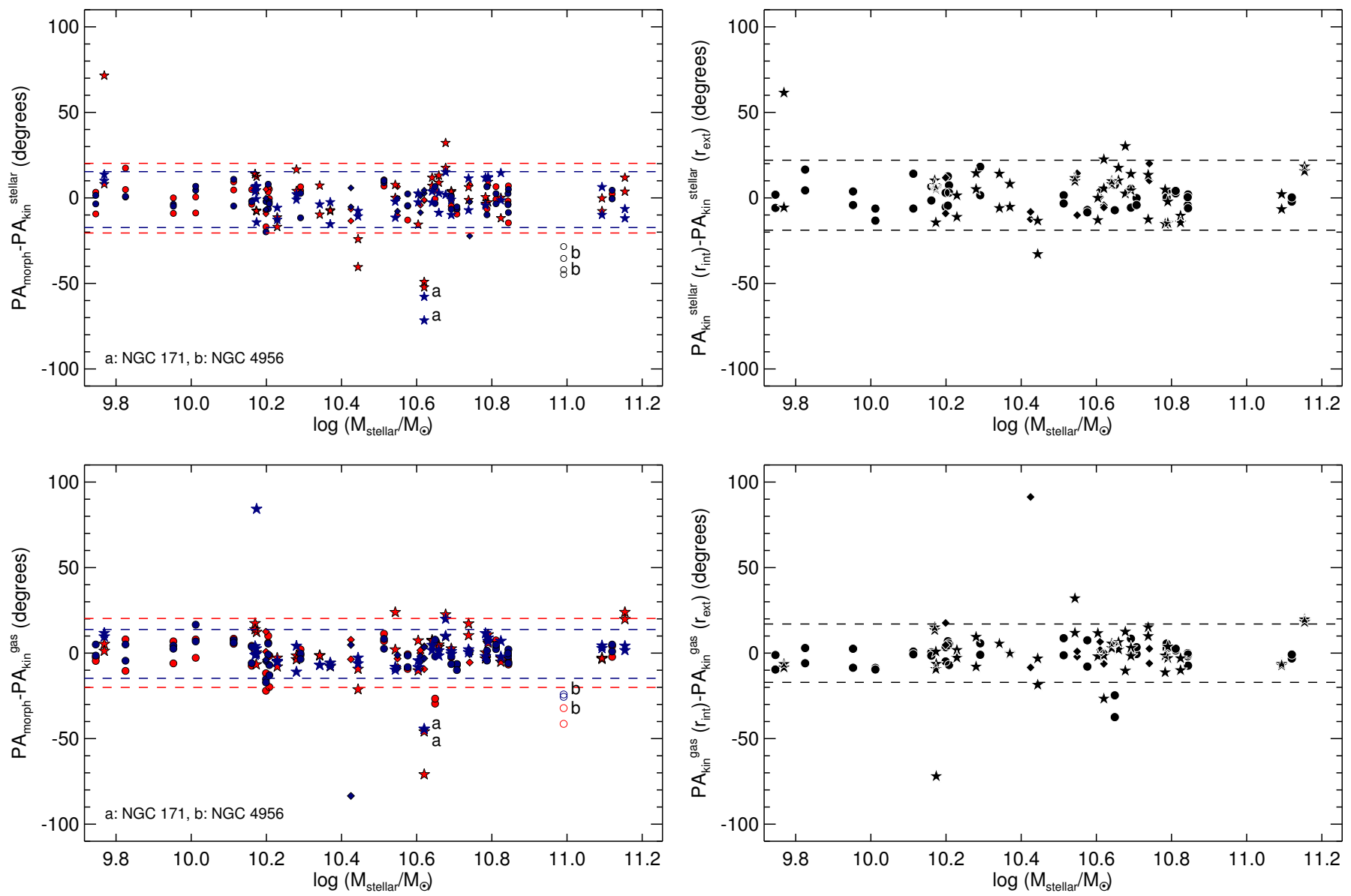

Fig. 4. Left panels: morpho-kinematic PA misalignments at different regions of the galaxy against the stellar mass for both the stellar (top panel) and the ionised gas (bottom panel) components in a subsample of low-inclined galaxies. In each of these panels, the red points represent the morpho-kinematic PA misalignment between PA morph (see Table B.1) and $\mathrm{PA}_{\text {kin }}$ up to $r_{\text {in }}$. Blue points represent the morpho-kinematic misalignment between $\mathrm{PA}_{\text {morph }}^{\text {out }}$ and $\mathrm{PA}_{\text {kin }}$ up to $r_{\text {out }}$ (see Sect. 4.3 for details). Filled circles, squares, and stars represent unbarred, weakly barred and strongly barred galaxies, respectively. Right panels: difference between the kinematic PA derived at $r_{\text {in }}$ and $r_{\text {out }}$ against the stellar mass for both the stellar (top panel) and the ionised gas (bottom panel) components. In the panels we highlight those objects with larger misalignments.

was measured within the same FoV probed by their kinematics (i.e., the inner regions of those galaxies). The $\mathrm{PA}_{\text {morph }}$ in those cases are thus biased due to the bar.

Throughout this study we find that the global kinematic orientation (measured by the major-axis kinematic PA) for the stellar and the ionised gas are aligned for non-interacting objects in the CALIFA survey (see Figs. 2 and 3). This result appears to hold at different radial lengths of the galaxies (Fig. 4), even for barred galaxies. To quantify the departures from perfect axisymmetry in the global kinematic orientation, we plot histograms in top panels of Fig. 5 showing the deviations from a straight line observed in the stellar and ionised-gas kinematic maps $\left(\delta \mathrm{PA}_{\mathrm{kin}}^{\text {stellar }}, \delta \mathrm{PA}_{\mathrm{kin}}^{\mathrm{gas}}\right)$. As already noted in Sect. 4.2 , the mean value of these deviations is higher for the stellar component than for the ionised gas. For both components the strongly barred galaxies present slightly higher values than the unbarred galaxies. In particular, the ionised gas component presents a difference between the distribution of barred and unbarred galaxies. This suggests that although the global kinematic orientation is stable in non-interacting galaxies, in particular for barred galaxies, some other kinematic parameters, such as $\delta \mathrm{PA}_{\mathrm{kin}}^{\text {gas }}$ (at higher spatial and spectral resolution as the present velocity fields ), can be sensitive to the presence of strong bars. It is important to note that there is still a fair fraction of strongly barred systems with $\delta \mathrm{PA}_{\text {kin }}$ consistent with those observed in unbarred galaxies. We have investigated whether this effect is caused by the flattening of the galaxy, or by the relative orientation of the bar with respect to the disc major axis. This is shown in the middle and lower panels of Fig. 5. The projected deviation of the kinemtic PA $\left(\delta \mathrm{PA}_{\mathrm{kin}}\right)$ seems to grow at lower apparent flattening of the galaxy (as a surrogate for inclination). This trend is particular clear for barred galaxies and may be induced by projections effects. Using the method presented in this study, any kinematic deviation from a straight line would be more easily quantified in a nearly face-on velocity field than in the same field viewed as edge-on. For highly inclined galaxies with large kinematic deviations, we may even trace vertical motions rather than radial deviation from a circular velocity field, however, other kinematic indicators, such as the intrinsic kinematic misalignment do not present a clear trend in ellipticity (see Appendix A). As for the orientation of the bar with respect to the disc major axis, the kinematic deviations covers a wide range of values for a given alignment between the bar and disc orientations, indicating that at least for barred galaxies, the orientation of the bar with respect to the disc may not affect drastically the scatter in the location of maximum velocities. 


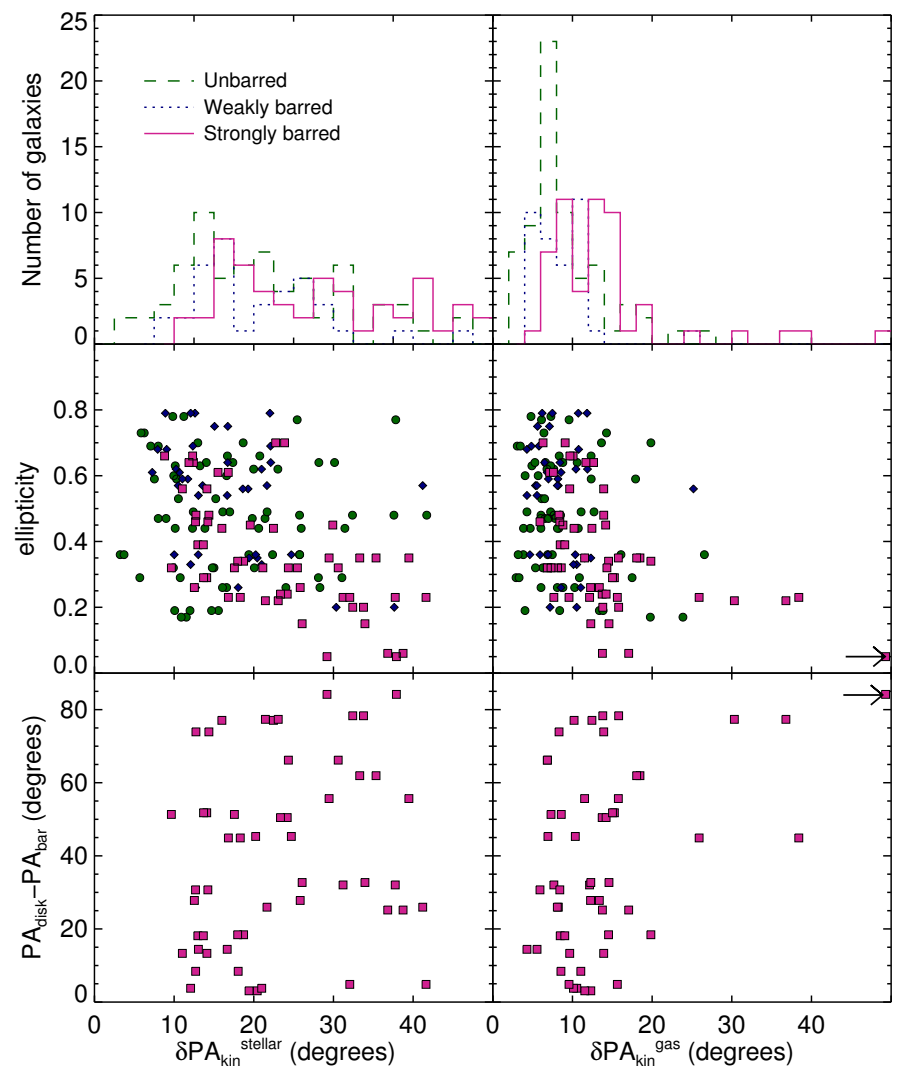

Fig. 5. Top: histograms of the kinematic PA deviations from a straight line in the velocity maps measured via $\delta \mathrm{PA}_{\text {kin }}^{\text {stellar }}(l e f t)$ and $\delta \mathrm{PA}_{\text {kin }}^{\text {gas }}(r i g h t)$ separated by different bar strengths. Dashed lines represent the unbarred galaxies, dotted lines correspond to the weakly barred galaxies and solid lines shows the distribution of strong barred galaxies. Middle: apparent flattening of the galaxies via the ellipticity, as in previous figures, the green filled circles represent the unbarred galaxies, blue diamonds correspond to weakly barred galaxies, and violet squares represent the barred galaxies. Bottom: difference between the PA of the disc $\left(\mathrm{PA}_{\text {disc }}\right)$ and the $\mathrm{PA}$ of the bar $\left(\mathrm{PA}_{\mathrm{bar}}\right)$ for strongly-barred galaxies alone. Arrows in middle and bottom gas panels indicate the galaxy $\operatorname{NGC} 171\left(\delta \mathrm{PA}_{\text {kin }}^{\text {gas }} \sim 90^{\circ}\right)$.

The velocity maps of barred galaxies often show, however, clear signatures of perturbations from a symmetric velocity field (e.g., Hernandez et al. 2005; Fathi et al. 2009). A characteristic "S"-shape in the zero-velocity curve is observed in several of these ionised gas kinematic maps (e.g., Peterson \& Huntley 1980; García-Barreto \& Rosado 2001; Emsellem et al. 2006). For a rotating disc galaxy, the major and minor axes are perpendicular everywhere (Binney \& Merrifield 1998). To account for the distortion in the zero-curve velocity, we estimate the difference between the above kinematic PAs. For each low-inclined galaxy $(\epsilon<0.5)$, we estimate the largest departure among the four kinematic PAs (approaching and receding major PAs and their corresponding minor PAs), where $0^{\circ}$ represents perpendicularity between major and minor axes. In Fig. 6 we represent these departures against the stellar mass (left panels) and the photometric alignment of the bar with respect to the disc for barred galaxies (right panels), for the stellar (top panels), and nebular components (bottom panels). For the stellar component, almost all the low-inclined galaxies present differences smaller than $\sim 30^{\circ}$ except for the barred galaxy NGC 6155. Even more, strongly-barred galaxies spread homogeneously in this range as the unbarred objects.

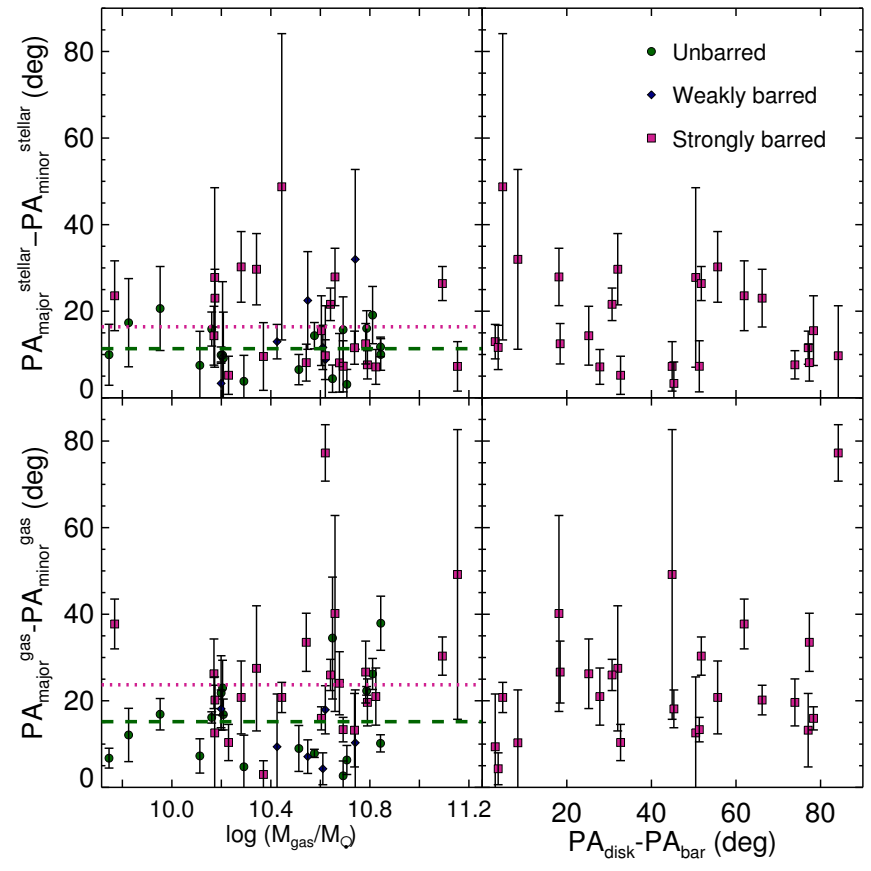

Fig. 6. Misalignment between the major and minor kinematic PA for a subsample of low-inclined galaxies $(\epsilon<0.5) .0^{\circ}$ represents perpendicularity between major and minor axes (see Sect. 5 for details). Left panels show these misalignments as function of the stellar mass for the stellar (top) and the ionised gas (bottom) components. Right panels present these kinematic misalignment as function of the morphological alignment of the bar with respect to the disc for barred galaxies. As in previous figures, the green-filled circles represent the unbarred galaxies, blue diamonds correspond to weakly barred galaxies and violet squares represent the barred galaxies. Dotted violet lines represent the mean value for the barred galaxies, while dashed-green lines represent the mean value for the unbarred galaxies.

The average departure between strongly and unbarred galaxies is similar $\left(\sim 11^{\circ}\right.$ and $\sim 15^{\circ}$, respectively). The ionised gas component displays a wide range of differences between the major and minor kinematic PAs. In particular, strongly barred galaxies present a wider range of differences than unbarred sample. The average of this difference for the barred galaxies is larger $\left(\sim 24^{\circ}\right)$ than the unbarred galaxies $\left(\sim 15^{\circ}\right)$. According to numerical simulations, the difference between the major and minor kinematic PAs is best seen in velocity fields where the bar makes an angle of $45^{\circ}$ with the major axis of the galaxy (e.g., Athanassoula 1984; Scannapieco \& Athanassoula 2012). Due to the moderate sub-sample of barred galaxies in this study and the rather large uncertainty in determining the minor kinematic PA, it is not clear whether larger differences in the kinematic PAs are observed at this specific angle between the bar and the disc PA. For the stellar component, however, an increment in this difference seems to be present at $\mathrm{PA}_{\text {disc }}-\mathrm{PA}_{\mathrm{bar}} \sim 50^{\circ}$. In the ionised gas, for a given morphological alignment between the bar and the disc we find a broad range of differences between the major and minor kinematic PAs. Given the spectral and spatial resolution in our velocities distributions, we consider that the difference between the major and minor kinematic PAs is a sensitive indicator of the presence of a bar in particular for the ionised gas component.

Detailed individual studies are required in order to explain the shape and length of the distortion in this velocity curve, as well as to determine if the ionised gas is more sensitive 
component to the bar potential. These issues are beyond the scope of this study; however, our results can motivate numerical simulations to address the nature of the kinematics in noninteracting galaxies. As opposed to previous indicators (see Figs. 2 and 3 ) this indicator of kinematic distortion varies significantly for non-interacting galaxies, making difficult to use it as a discriminator for distinguishing the kinematic distortion produced by the merging event or a secular process.

\section{Conclusions}

We studied the stellar and ionised-gas kinematics for a sample of 80 non-interacting galaxies in the CALIFA survey. The fraction of barred and unbarred objects in this sample is similar. We found that for $90 \%$ of the sample the global orientation of the stellar and ionised gas is fairly well aligned (misalignment smaller than $\sim 20^{\circ}$ ) with respect to the global photometric orientation of the galaxy, including barred and unbarred objects. From the method used to measure the major kinematic PA, we studied the internal kinematic PA misalignments namely, the difference between the receding and approaching kinematic PA. We found intrinsic aligned velocity fields in both components for a large fraction of the sample (intrinsic misalignments smaller than $15^{\circ}$ for the stellar and the ionised gas components).

We also compared the derived kinematic PA for the stellar and the ionised gas components. We observe a tight alignment ( $16^{\circ}$ of difference) between both components for the majority of this non-interacting sample. This result holds in barred galaxies when the comparison is carried out either at the radius of the bar or further out. From these results we suggest that the global kinematics in non-interacting galaxies in both the stellar and the ionised gas components (measured by the major kinematic position angle) seems to be dominated by the mass of the disc, rather than other morphological components that can induce non-circular patterns in the observed velocity fields, such as bars. Even though bars can locally redistribute the angular momentum, energy, and mass; the kinematic pattern that dominates across the galactic disc - including the bar - is consistent with a regular rotational pattern.

We will use the results presented in this paper to gauge the kinematic distortions caused by external forces in galaxies that undergo a merger event in Barrera-Ballesteros et al. (in prep.).

Acknowledgements. We thank the referee for a thorough reading of this work and for the comments and suggestions. We also thank Lindsay Holmes for allowing us to use her DiskFit kinematic modelling for comparison with our results. This study makes use of the data provided by the Calar Alto Legacy Field Area (CALIFA) survey (http://www.califa.caha.es), and is based on observations collected at the Centro Astronómico Hispano Alemán (CAHA) at Calar Alto, operated jointly by the Max-Planck-Institut für Astronomie and the Instituto de Astrofísica de Andalucia (CSIC). CALIFA is the first legacy survey performed at Calar Alto. The CALIFA collaboration would like to thanks the IAA-CSIC and MPIA-MPG as major partners of the observatory, and CAHA itself for the unique access to telescope time and for support in manpower and infrastructures. The CALIFA collaboration also thanks the CAHA staff for their dedication to this project. J.B.-B. and B.G.-L. acknowledge the support from the Plan Nacional de I+D+i (PNAYA) funding programme (AYA201239408-C02-02) of the Spanish Ministry of Economy and Competitiveness (MINECO). J.F.-B. acknowledges support from the Ramón y Cajal Program, grants AYA2010-21322-C03-02 from MINECO. We also acknowledge support from the FP7 Marie Curie Actions of the European Commission, via the Initial Training Network DAGAL under REA grant agreement number 289313. R.A.M is funded by the Spanish programme of International Campus of Excellence Moncloa (CEI). L.V.M. acknowledges support from the grant AYA201130491-C02-01 co-financed by MICINN and FEDER funds, and the Junta de Andalucia (Spain) grants P08-FQM-4205 and TIC-114. J.I.P. acknowledges financial support from the Spanish MINECO under grant AYA2010-21887-C0401 and from Junta de Andalucía Excellence Project PEX2011-FQM7058. J.M.A. acknowledges support from the European Research Council Starting Grant (SEDmorph; P.I. V. Wild).

\section{References}

Abazajian, K. N., Adelman-McCarthy, J. K., Agüeros, M. A., et al. 2009, ApJS, 182,543

Aguerri, J. A. L., Méndez-Abreu, J., \& Corsini, E. M. 2009, A\&A, 495, 491

Athanassoula, E. 1984, Phys. Rep., 114, 319

Athanassoula, E. 2003, MNRAS, 341, 1179

Barazza, F. D., Jogee, S., \& Marinova, I. 2008, ApJ, 675, 1194

Barnes, E. I., \& Sellwood, J. A. 2003, AJ, 125, 1164

Binney, J., \& Merrifield, M. 1998, Galactic Astronomy (Princeton: Princeton University Press)

Binney, J., \& Tremaine, S. 2008, Galactic Dynamics, 2nd edn. (Princeton University Press)

Cappellari, M., \& Copin, Y. 2003, MNRAS, 342, 345

Cappellari, M., \& Emsellem, E. 2004, PASP, 116, 138

Cappellari, M., Emsellem, E., Krajnović, D., et al. 2011, MNRAS, 413, 813

Croom, S. M., Lawrence, J. S., Bland-Hawthorn, J., et al. 2012, MNRAS, 421, 872

Debattista, V. P., \& Sellwood, J. A. 1998, ApJ, 493, L5

Debattista, V. P., \& Sellwood, J. A. 2000, ApJ, 543, 704

Ellison, S. L., Patton, D. R., Simard, L., \& McConnachie, A. W. 2008, AJ, 135, 1877

Emsellem, E., Cappellari, M., Peletier, R. F., et al. 2004, MNRAS, 352, 721

Emsellem, E., Fathi, K., Wozniak, H., et al. 2006, MNRAS, 365, 367

Epinat, B., Amram, P., Marcelin, M., et al. 2008, MNRAS, 388, 500

Eskridge, P. B., Frogel, J. A., Pogge, R. W., et al. 2000, AJ, 119, 536

Falcón-Barroso, J., Bacon, R., Bureau, M., et al. 2006, MNRAS, 369, 529

Fathi, K., van de Ven, G., Peletier, R. F., et al. 2005, MNRAS, 364, 773

Fathi, K., Beckman, J. E., Piñol-Ferrer, N., et al. 2009, ApJ, 704, 1657

García-Barreto, J. A., \& Rosado, M. 2001, AJ, 121, 2540

García-Lorenzo, B. 2013, MNRAS, 534

García-Lorenzo, B., Márquez, I., Barrera-Ballesteros, J. K. et al. 2014, A\&A, accepted

Hernandez, O., Carignan, C., Amram, P., Chemin, L., \& Daigle, O. 2005, MNRAS, 360, 1201

Hernandez, O., Fathi, K., Carignan, C., et al. 2008, PASP, 120, 665

Husemann, B., Jahnke, K., Sánchez, S. F., et al. 2013, A\&A, 549, A87

Józsa, G. I. G., Kenn, F., Klein, U., \& Oosterloo, T. A. 2007, A\&A, 468, 731

Knapen, J. H., Shlosman, I., \& Peletier, R. F. 2000, ApJ, 529, 93

Krajnović, D., Cappellari, M., de Zeeuw, P. T., \& Copin, Y. 2006, MNRAS, 366, 787

Krajnović, D., Emsellem, E., Cappellari, M., et al. 2011, MNRAS, 414, 2923

Kronberger, T., Kapferer, W., Unterguggenberger, S., Schindler, S., \& Ziegler, B. L. 2008, A\&A, 483, 783

Kutdemir, E., Ziegler, B. L., Peletier, R. F., et al. 2008, A\&A, 488, 117

Marinova, I., Jogee, S., Bacon, D., et al. 2007, BAAS, 39, 905

Martinez-Valpuesta, I., Shlosman, I., \& Heller, C. 2006, ApJ, 637, 214

Menéndez-Delmestre, K., Sheth, K., Schinnerer, E., Jarrett, T. H., \& Scoville, N. Z. 2007, ApJ, 657, 790

Moreno, J., Bluck, A. F. L., Ellison, S. L., et al. 2013, MNRAS, 436, 1765

Naab, T., \& Burkert, A. 2003, ApJ, 597, 893

Nicholson, R. A., Bland-Hawthorn, J., \& Taylor, K. 1992, ApJ, 387, 503

Peterson, C. J., \& Huntley, J. M. 1980, ApJ, 242, 913

Roth, M. M., Kelz, A., Fechner, T., et al. 2005, PASP, 117, 620

Rozas, M., Relaño, M., Zurita, A., \& Beckman, J. E. 2002, A\&A, 386, 42

Sánchez, S. F., Kennicutt, R. C., Gil de Paz, A., et al. 2012, A\&A, 538, A8

Scannapieco, C., \& Athanassoula, E. 2012, MNRAS, 425, L10

Scannapieco, C., Gadotti, D. A., Jonsson, P., \& White, S. D. M. 2010, MNRAS 407, L41

Schoenmakers, R. H. M., Franx, M., \& de Zeeuw, P. T. 1997, MNRAS, 292, 349

Sellwood, J. A. 2006, ApJ, 637, 567

Sellwood, J. A. 2013, in Dynamics of Disks and Warps, eds. T. D. Oswalt, \& G. Gilmore (Springer Science + Business Media Durdrecht), 923

Sellwood, J. A., \& Debattista, V. P. 2006, ApJ, 639, 868

Sofue, Y., \& Rubin, V. 2001, ARA\&A, 39, 137

Spekkens, K., \& Sellwood, J. A. 2007, ApJ, 664, 204

Thielheim, K. O., \& Wolff, H. 1982, MNRAS, 199, 151

Valdes, F., Gupta, R., Rose, J. A., Singh, H. P., \& Bell, D. J. 2004, ApJS, 152, 251

Varela, J., Moles, M., Márquez, I., et al. 2004, A\&A, 420, 873

Verdes-Montenegro, L., Sulentic, J., Lisenfeld, U., et al. 2005, A\&A, 436, 443

Verley, S., Leon, S., Verdes-Montenegro, L., et al. 2007, A\&A, 472, 121 
Villa-Vargas, J., Shlosman, I., \& Heller, C. 2009, ApJ, 707, 218

Walcher, C. J., Wisotzki, L., Bekeraité, S., et al. 2014, A\&A, in press DOI: $10.1051 / 0004-6361 / 201424198$

Weinberg, M. D. 1985, MNRAS, 213, 451

1 Instituto de Astrofísica de Canarias, Calle vía Lactéa s/n, 38205, Spain

e-mail: jkbb@iac.es

2 Departamento de Astrofísica, Universidad de La Laguna (ULL), 38200 La Laguna, Tenerife, Spain

3 Max-Planck Institute for Astronomy, Königstul 17, 69117 Heidelberg, Germany

4 School of Physics and Astronomy, University of St Andrews, North Haugh, St Andrews, KY16 9SS, UK

5 Department of Physics, Royal Military Collage of Canada, PO Box 17000, Station Forces, Kingston, Ontario, K7K7B4, Canada

${ }^{6}$ Instituto de Astrofísica de Andalucía (CSIC), Glorieta de la Astronomía S/N, 18008 Granada, Spain
7 Instituto de Astronomía, Universidad Nacional Autonóma de Mexico, A.P. 70-264, 04510 México, D.F., Mexico

8 Centro Astronómico Hispano Alemán de Calar Alto (CSIC-MPG), C/ Jesús Durbán Remón 2-2, 4004 Almería, Spain

9 European Southern Observatory, ESO Headquarters, KarlSchwarzschild-Str. 2, 85748 Garching b. München, Germany

${ }^{10}$ Leibniz-Institut für Astrophysik Potsdam (AIP), An der Sternwarte 16, 14482 Potsdam, Germany

11 CEI Campus Moncloa, UCM-UPM, Departamento de Astrofísica y CC. de la Atmósfera, Facultad de CC. Físicas, Universidad Complutense de Madrid, Avda. Complutense S/N, 28040 Madrid, Spain

12 University of Vienna, Department of Astrophysics, Türkenschanzstr. 17, 1180 Vienna, Austria

13 Department of Physics \& Astronomy, University of MissouriKansas City, 5110 Rockhill Road, Kansas City, MO, USA

14 Sydney Institute for Astronomy, School of Physics A28, University of Sydney, NSW 2006 Sydney, Australia 


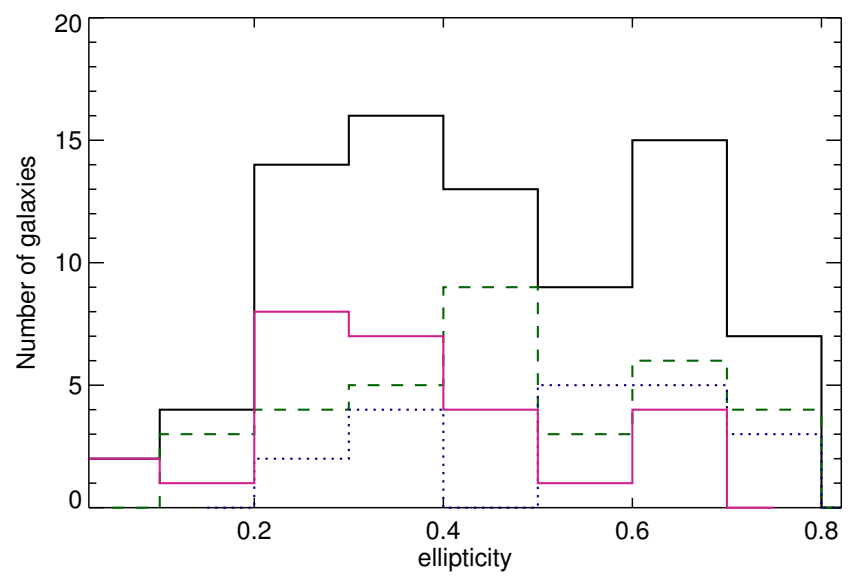

Fig. A.1. Distribution of the ellipticity as proxy of the inclination for the galaxies used in this study. Dashed lines represent the unbarred galaxies, dotted-lines correspond to the weakly barred galaxies and solid lines shows the distribution of strong barred galaxies.

\section{Appendix A: Kinematic alignments versus apparent ellipticity}

All the kinematic parameters presented in this study are determined on the plane of the sky. Therefore, it is expected that any intrinsic kinematic property would be affected by the inclination of the galaxy with respect to the plane of the sky. In this appendix we show the same kinematic misalignments presented in Sect. 4 with respect to the apparent ellipticity as proxy of the apparent inclination of the galaxies.

In Fig. A.1 we plot the distribution of the non-interacting galaxies with respect to the ellipticity. We have a fair coverage of galaxies at different inclinations, in particular for the lowinclined ones $(\epsilon<0.5)$. To have consistent results, we also try to have the same fraction of barred vs. unbarred galaxies in different ellipticity bins.

In Fig. A. 2 we plot the morpho-kinematic misalignments explained in Sect. 4.1 against the ellipticity for both components, as well as the internal kinematic misalignment in each component (Fig. A.3) and the comparison of the kinematic PA between the stars and the gas (Fig. A.4). We find large morpho-kinematic misalignments in galaxies with low ellipticities $(\epsilon<0.3$, see Fig. A.2).

For the kinematic parameters independent of the morphology (i.e., Figs. A.3 and A.4), we find rather similar values in different ellipticity bins. In each panel of these figures, we plot an estimation of the projections effects as function of the ellipticity assuming a face-on misalignment of $60^{\circ}$ (see Fig. A.2) in
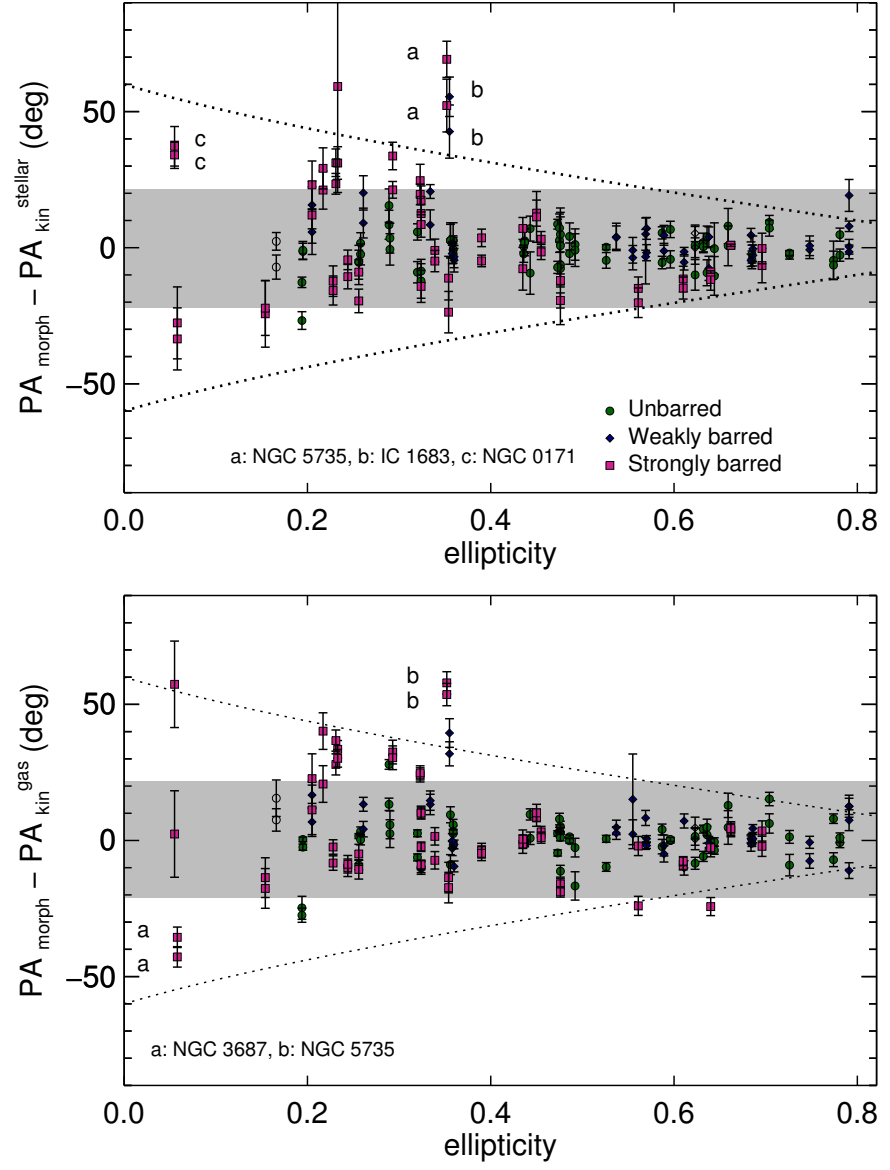

Fig. A.2. Difference between the morphological $\mathrm{PA}\left(\mathrm{PA}_{\text {morph }}\right)$ and the kinematic $\mathrm{PA}\left(\mathrm{PA}_{\mathrm{kin}}\right)$ against the ellipticity of the outermost isophote of the galaxy for the stars (top panel) and the ionised gas (bottom panel). Both kinematic sides of the velocity maps were compared with $\mathrm{PA}_{\text {morph }}$. In each panel, the green filled circles represent the unbarred galaxies, blue diamonds are weakly barred galaxies, and violet squares correspond to barred galaxies. For early-type galaxies we use empty circles. Labels indicate the $3 \sigma$ outliers. Dashed lines represent the projection effect of a morpho-kinematic misalignment of $60^{\circ}$ measured in the plane of the galaxy. In each panel, the grey area represents the $2 \sigma$ dispersion of the sample.

any of the (morpho-)kinematic indicators. This value was chosen to approximately match the misalignments found at large ellipticities. Within uncertainties, all the galaxies display internal kinematic misalignments that are smaller than the ones expected for their inclinations. In other words, for these misalignments, we do not find large scatters at low ellipticities, as expected for a quantity heavily affected by projection effects. 

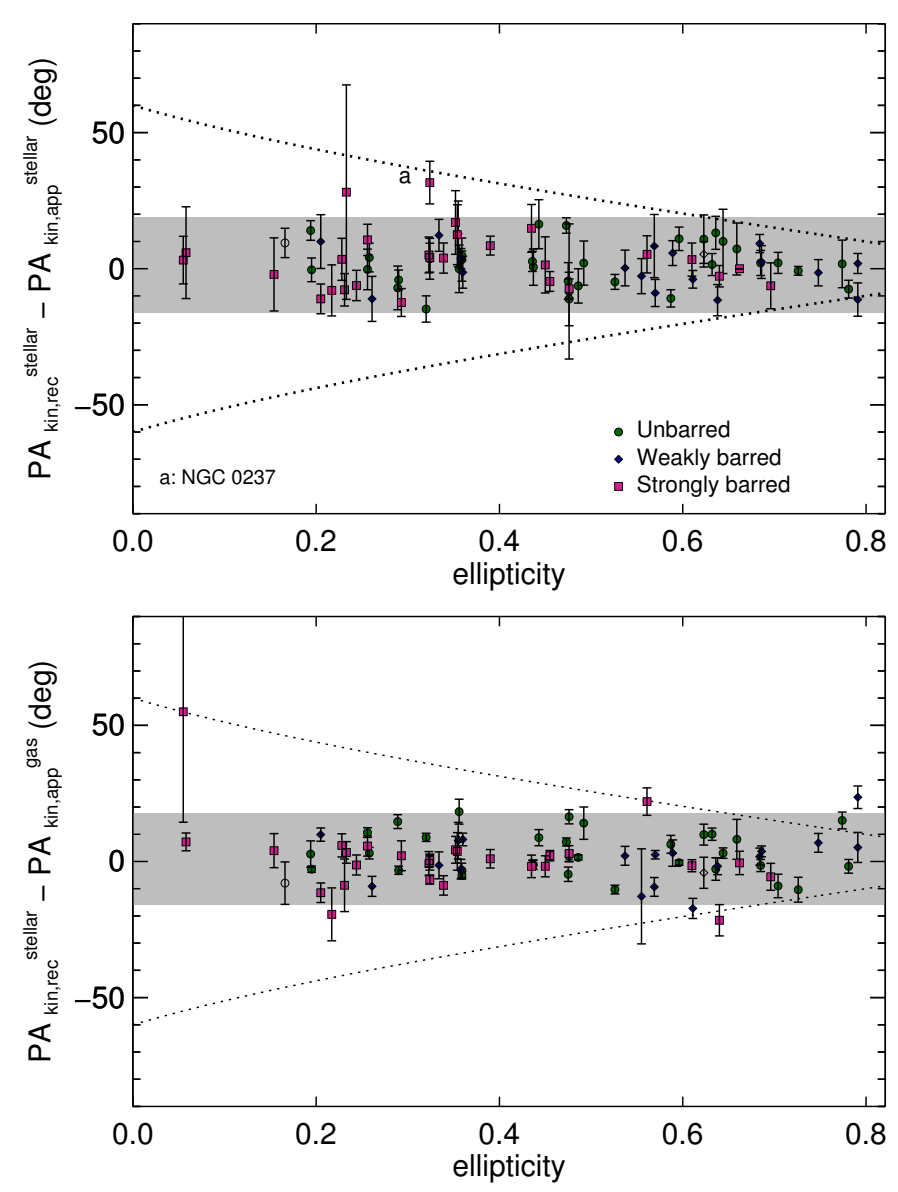

Fig. A.3. Internal kinematic misalignment between the receding PA $\left(\mathrm{PA}_{\mathrm{kin}, \mathrm{rec}}\right)$ and approaching $\left(\mathrm{PA}_{\mathrm{kin}, a p p}\right)$ kinematic position angle against the ellipticity. In each panel the green filled circles represent the unbarred galaxies, blue diamonds correspond to weakly barred galaxies, and violet squares represent the barred galaxies. Empty circles represent the early type galaxies. Labels indicate the $3 \sigma$ outlier. Dashed lines represent the projection effect of a morpho-kinematic misalignment of $60^{\circ}$ measured in the plane of the galaxy. In each panel, the grey area represents the $2 \sigma$ dispersion of the sample.

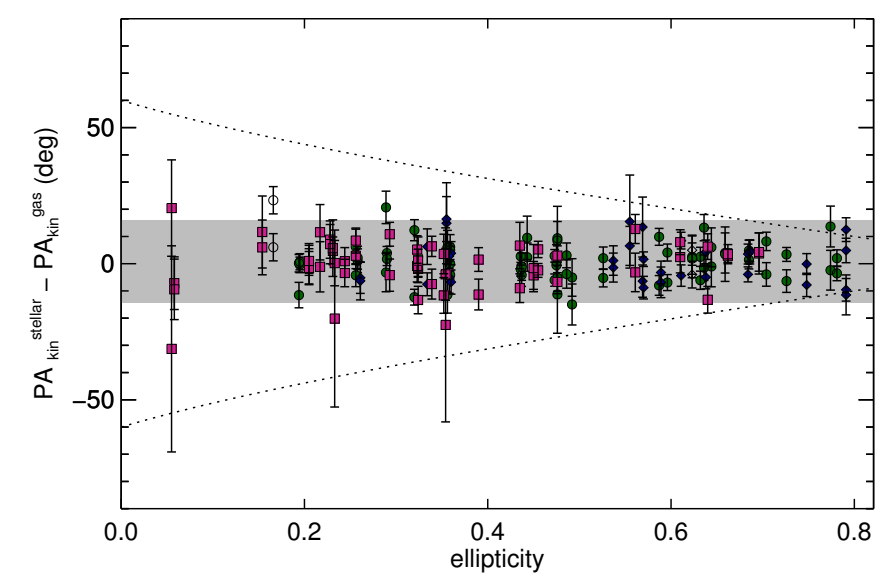

Fig. A.4. Stellar and ionised gas difference respects the ellipticity. The green filled circles represent the unbarred galaxies, blue diamonds correspond to weakly barred galaxies, and violet squares represent the barred galaxies. Empty circles represent the early-type galaxies. Dashed lines represent the projection effect of a morpho-kinematic misalignment of $60^{\circ}$ measured in the plane of the galaxy. In each panel, the grey area represents the $2 \sigma$ dispersion of the sample. 
J. K. Barrera-Ballesteros et al.: Kinematic alignment of non-interacting CALIFA galaxies

\section{Appendix B: Tables}

Table B.1. Morphological parameters of the sample of non-interacting CALIFA galaxies used in this study.

\begin{tabular}{|c|c|c|c|c|c|c|c|c|}
\hline $\begin{array}{c}\text { CALIFA id } \\
\text { (1) }\end{array}$ & $\begin{array}{c}\text { Name } \\
(2) \\
\end{array}$ & $\begin{array}{l}\text { Morphological } \\
\text { type } \\
(3)\end{array}$ & $\begin{array}{c}\text { Bar } \\
\text { strength } \\
(4)\end{array}$ & $\begin{array}{r}\epsilon \\
(5) \\
\end{array}$ & $\begin{array}{c}\mathrm{PA}_{\text {morph }}^{\text {out }} \\
\left({ }^{\circ}\right) \\
(6) \\
\end{array}$ & $\begin{array}{c}\mathrm{PA}_{\text {bar }} \\
\left({ }^{\circ}\right) \\
(7) \\
\end{array}$ & $\begin{array}{c}r_{\mathrm{bar}} \\
(\operatorname{arcsec}) \\
(8)\end{array}$ & $\begin{array}{c}\text { Stellar mass } \\
\log \left(M / M_{\odot}\right) \\
(9) \\
\end{array}$ \\
\hline 1 & IC 5376 & $\mathrm{Sb}$ & $\mathrm{A}$ & 0.69 & 50 & $\ldots$ & $\ldots$ & 10.57 \\
\hline 2 & UGC 00005 & Sbc & A & 0.53 & 50 & $\ldots$ & $\ldots$ & 10.72 \\
\hline 7 & UGC 00036 & $\mathrm{Sab}$ & $\mathrm{AB}$ & 0.61 & 50 & $\ldots$ & $\ldots$ & 10.88 \\
\hline 8 & NGC 0001 & Sbc & A & 0.32 & 50 & $\ldots$ & $\ldots$ & 10.71 \\
\hline 10 & NGC 0036 & $\mathrm{Sb}$ & $\mathrm{B}$ & 0.48 & 50 & $\ldots$ & $\dddot{12}$ & 10.79 \\
\hline 23 & NGC 0171 & $\mathrm{Sb}$ & B & 0.05 & 50 & 122 & 33 & 10.62 \\
\hline 25 & NGC 0180 & $\mathrm{Sb}$ & $\mathrm{B}$ & 0.34 & 50 & 147 & 22 & 10.78 \\
\hline 28 & NGC 0214 & Sbc & $\mathrm{AB}$ & 0.26 & 50 & 59 & 14 & 10.74 \\
\hline 30 & NGC 0237 & $\mathrm{Sc}$ & $\mathrm{B}$ & 0.32 & 50 & 40 & 7 & 10.17 \\
\hline 31 & NGC 0234 & $\mathrm{Sc}$ & $\mathrm{AB}$ & 0.20 & 50 & $\ldots$ & $\ldots$ & 10.55 \\
\hline 33 & NGC 0257 & $\mathrm{Sc}$ & A & 0.36 & 50 & $\ldots$ & $\ldots$ & 10.69 \\
\hline 41 & UGC 00841 & $\mathrm{Sbc}$ & $\mathrm{A}$ & 0.77 & 50 & $\ldots$ & $\ldots$ & 9.71 \\
\hline 43 & IC 1683 & $\mathrm{Sb}$ & $\mathrm{AB}$ & 0.35 & 50 & 168 & 8 & 10.43 \\
\hline 53 & UGC 01057 & $\mathrm{Sc}$ & $\mathrm{AB}$ & 0.69 & 50 & $\ldots$ & $\ldots$ & 10.11 \\
\hline 108 & NGC 1093 & $\mathrm{Sbc}$ & B & 0.39 & 50 & 117 & 10 & 10.66 \\
\hline 116 & UGC 02405 & Sbc & $\mathrm{A}$ & 0.66 & 50 & $\ldots$ & $\ldots$ & 10.40 \\
\hline 131 & NGC 1542 & $\mathrm{Sab}$ & $\mathrm{AB}$ & 0.59 & 50 & $\begin{array}{l}\cdots \\
\cdots\end{array}$ & $\begin{array}{l}\cdots \\
\cdots\end{array}$ & 10.30 \\
\hline 147 & NGC 2253 & Sbc & $\mathrm{B}$ & 0.32 & 50 & $\begin{array}{l}\cdots \\
\cdots\end{array}$ & $\begin{array}{l}\cdots \\
\cdots\end{array}$ & 10.37 \\
\hline 149 & NGC 2347 & Sbc & $\mathrm{AB}$ & 0.36 & 50 & $\begin{array}{l}\cdots \\
\cdots\end{array}$ & $\begin{array}{l}\cdots \\
\cdots\end{array}$ & 10.62 \\
\hline 151 & NGC 2410 & $\mathrm{Sb}$ & $\mathrm{AB}$ & 0.68 & 50 & $\ldots$ & $\ldots$ & 10.78 \\
\hline 152 & UGC 03944 & $\mathrm{Sbc}$ & $\mathrm{AB}$ & 0.57 & 50 & 94 & 5 & 9.87 \\
\hline 153 & UGC 03969 & $\mathrm{Sb}$ & A & 0.78 & 50 & $\ldots$ & $\ldots$ & 10.61 \\
\hline 273 & IC 2487 & $\mathrm{Sc}$ & $\mathrm{AB}$ & 0.79 & 50 & $\ldots$ & $\ldots$ & 10.29 \\
\hline 275 & NGC 2906 & Sbc & A & 0.44 & 50 & $\cdots$ & $\begin{array}{l}\cdots \\
\cdots\end{array}$ & 10.29 \\
\hline 277 & NGC 2916 & Sbc & A & 0.36 & 50 & $\ldots$ & $\ldots$ & 10.65 \\
\hline 307 & UGC 05359 & $\mathrm{Sb}$ & B & 0.70 & 50 & $\ldots$ & $\ldots$ & 10.54 \\
\hline 364 & UGC 06036 & $\mathrm{Sa}$ & A & 0.73 & 50 & $\cdots$ & $\cdots$ & 11.09 \\
\hline 386 & UGC 06312 & $\mathrm{Sab}$ & A & 0.64 & 50 & $\ldots$ & $\ldots$ & 10.93 \\
\hline 414 & NGC 3687 & $\mathrm{Sb}$ & $\mathrm{B}$ & 0.06 & 50 & 174 & 14 & 10.17 \\
\hline 436 & NGC 3811 & Sbc & B & 0.23 & 50 & 22 & 24 & 10.34 \\
\hline 489 & NGC 4047 & Sbc & $\mathrm{A}$ & 0.26 & 50 & $\ldots$ & $\ldots$ & 10.58 \\
\hline 515 & NGC 4185 & Sbc & $\mathrm{AB}$ & 0.33 & 50 & 167 & 20 & 10.61 \\
\hline 518 & NGC 4210 & $\mathrm{Sb}$ & B & 0.24 & 50 & 47 & 17 & 10.17 \\
\hline 580 & NGC 4711 & $\mathrm{Sbc}$ & A & 0.47 & 50 & $\ldots$ & $\ldots$ & 10.20 \\
\hline 602 & NGC 4956 & E1 & $\mathrm{A}$ & 0.17 & 50 & $\ldots$ & $\ldots$ & 10.99 \\
\hline 610 & UGC 08267 & $\mathrm{Sb}$ & $\mathrm{AB}$ & 0.75 & 50 & $\ldots$ & $\ldots$ & 10.68 \\
\hline 624 & NGC 5157 & $\mathrm{Sab}$ & $\mathrm{B}$ & 0.23 & 50 & 138 & 21 & 11.15 \\
\hline 630 & NGC 5205 & Sbc & $\mathrm{B}$ & 0.35 & 50 & 108 & 12 & 9.77 \\
\hline 664 & UGC 08778 & $\mathrm{Sb}$ & A & 0.70 & 50 & $\ldots$ & $\ldots$ & 10.15 \\
\hline 684 & NGC 5406 & $\mathrm{Sb}$ & $\mathrm{B}$ & 0.29 & 50 & 56 & 20 & 11.09 \\
\hline 714 & UGC 09067 & $\mathrm{Sbc}$ & $\mathrm{AB}$ & 0.54 & 50 & 27 & 5 & 10.36 \\
\hline 715 & NGC 5520 & Sbc & $\mathrm{A}$ & 0.49 & 50 & $\ldots$ & $\ldots$ & 9.75 \\
\hline 743 & NGC 5622 & $\mathrm{Sbc}$ & A & 0.48 & 50 & $\begin{array}{l}\cdots \\
\cdots\end{array}$ & $\begin{array}{l}\cdots \\
\cdots\end{array}$ & 10.11 \\
\hline 748 & NGC 5633 & Sbc & A & 0.26 & 50 & $\begin{array}{l}\cdots \\
\ldots\end{array}$ & $\begin{array}{l}\cdots \\
\ldots\end{array}$ & 10.16 \\
\hline 753 & NGC 5656 & $\mathrm{Sb}$ & A & 0.19 & 50 & $\begin{array}{l}\cdots \\
\ldots\end{array}$ & $\begin{array}{l}\cdots \\
\ldots\end{array}$ & 10.51 \\
\hline 764 & NGC 5720 & Sbc & $\mathrm{B}$ & 0.44 & 50 & $\ldots 0$ & $\dddot{7}$ & 10.74 \\
\hline 768 & NGC 5732 & $\mathrm{Sbc}$ & A & 0.48 & 50 & $\ldots$ & $\ldots$ & 9.83 \\
\hline 771 & NGC 5735 & Sbc & $\mathrm{B}$ & 0.35 & 50 & $\dddot{94}$ & 20 & 10.28 \\
\hline 777 & NGC 5772 & $\mathrm{Sab}$ & $\mathrm{A}$ & 0.44 & 50 & $\ldots$ & $\ldots$ & 10.84 \\
\hline 779 & UGC 09598 & Sbc & $\mathrm{AB}$ & 0.64 & 50 & $\ldots$ & $\ldots$ & 10.45 \\
\hline 782 & UGC 09629 & E7 & $\mathrm{AB}$ & 0.62 & 50 & $\begin{array}{l}\cdots \\
\cdots\end{array}$ & $\begin{array}{l}\cdots \\
\cdots\end{array}$ & 11.02 \\
\hline
\end{tabular}

Notes. (1) CALIFA ID number. (2) Name of the galaxy. (3) Morphological type from visual clasification (see Walcher et al. 2014, for details). (4) Bar strength of the galaxy from the same morphological visual clasification (see Walcher et al. 2014, for details). (5) and (6) ellipticity ( $\epsilon$ ) and position angle $\left(\mathrm{PA}_{\mathrm{morph}}^{\text {out }}\right)$ from an ellipse fitting at the largest scale isophote of the SDSS $r$-band image. Both measurements were inferred using the IRAF task ellipse. (7) and (8) Position angle $\left(\mathrm{PA}_{\mathrm{bar}}\right)$ and length of the bar $\left(r_{\mathrm{bar}}\right)$ derived from an ellipse fitting on the SDSS $r$-band image. (9) Stellar masses (see Walcher et al. 2014, for details). 
Table B.1. continued.

\begin{tabular}{|c|c|c|c|c|c|c|c|c|}
\hline CALIFA id & Name & $\begin{array}{l}\text { Morphological } \\
\text { type } \\
\text { (3) }\end{array}$ & Barredness & $(5)$ & $\begin{array}{l}\mathrm{PA}_{\text {morph }}^{\text {out }} \\
\left(^{\circ}\right) \\
(6)\end{array}$ & $\begin{array}{l}\mathrm{PA}_{\mathrm{bar}} \\
\left({ }^{\circ}\right) \\
(7)\end{array}$ & $\begin{array}{c}r_{\mathrm{bar}} \\
(\operatorname{arcsec}) \\
(8)\end{array}$ & $\begin{array}{c}\text { Stellar mass } \\
\log \left(M / M_{\odot}\right) \\
(9)\end{array}$ \\
\hline 790 & UGC 09777 & $\mathrm{Sbc}$ & A & 0.49 & 50 & $\ldots$ & $\ldots$ & 10.21 \\
\hline 791 & NGC 5908 & $\mathrm{Sa}$ & A & 0.36 & 50 & $\ldots$ & $\ldots$ & 11.12 \\
\hline 804 & NGC 5971 & $\mathrm{Sb}$ & $\mathrm{AB}$ & 0.56 & 50 & $\ldots$ & $\ldots$ & 10.22 \\
\hline 810 & NGC 5980 & $\mathrm{Sbc}$ & $\mathrm{A}$ & 0.60 & 50 & $\ldots$ & $\ldots$ & 10.62 \\
\hline 813 & NGC 6004 & $\mathrm{Sbc}$ & B & 0.20 & 50 & 15 & 16 & 10.60 \\
\hline 823 & NGC 6063 & $\mathrm{Sbc}$ & A & 0.44 & 50 & $\ldots$ & $\ldots$ & 9.95 \\
\hline 824 & IC 1199 & $\mathrm{Sb}$ & $\mathrm{AB}$ & 0.57 & 50 & $\ldots$ & $\ldots$ & 10.20 \\
\hline 826 & NGC 6081 & S0a & A & 0.59 & 50 & $\ldots$ & $\ldots$ & 11.04 \\
\hline 831 & NGC 6132 & $\mathrm{Sbc}$ & A & 0.64 & 50 & $\ldots$ & $\ldots$ & 10.15 \\
\hline 834 & UGC 10380 & $\mathrm{Sb}$ & $\mathrm{AB}$ & 0.79 & 50 & $\ldots$ & $\ldots$ & 10.92 \\
\hline 836 & NGC 6155 & $\mathrm{Sc}$ & A & 0.29 & 50 & $\ldots$ & $\ldots$ & 10.01 \\
\hline 842 & NGC 6186 & $\mathrm{Sb}$ & B & 0.23 & 50 & 54 & 35 & 10.44 \\
\hline 853 & NGC 6361 & Sab & A & 0.29 & 50 & $\ldots$ & $\ldots$ & 10.81 \\
\hline 854 & UGC 10811 & $\mathrm{Sb}$ & B & 0.66 & 50 & $\ldots$ & $\ldots$ & 10.75 \\
\hline 856 & IC 1256 & $\mathrm{Sb}$ & $\mathrm{AB}$ & 0.36 & 50 & 130 & 7 & 10.20 \\
\hline 857 & NGC 6394 & Sbc & B & 0.64 & 50 & $\ldots$ & $\ldots$ & 10.79 \\
\hline 862 & NGC 6478 & $\mathrm{Sc}$ & A & 0.63 & 50 & 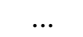 & $\ldots$ & 10.90 \\
\hline 869 & NGC 6941 & $\mathrm{Sb}$ & B & 0.26 & 50 & 108 & 16 & 10.82 \\
\hline 872 & UGC 11649 & Sab & B & 0.22 & 50 & 162 & 17 & 10.54 \\
\hline 876 & NGC 7047 & Sbc & B & 0.45 & 50 & 115 & 20 & 10.68 \\
\hline 886 & NGC 7311 & $\mathrm{Sa}$ & A & 0.47 & 50 & $\ldots$ & $\ldots$ & 10.84 \\
\hline 887 & NGC 7321 & Sbc & B & 0.32 & 50 & 62 & 10 & 10.69 \\
\hline 889 & NGC 7364 & Sab & A & 0.32 & 50 & $\ldots$ & $\ldots$ & 10.79 \\
\hline 890 & UGC 12185 & $\mathrm{Sb}$ & B & 0.56 & 50 & 139 & 22 & 10.58 \\
\hline 896 & NGC 7466 & Sbc & A & 0.62 & 50 & $\ldots$ & $\ldots$ & 10.70 \\
\hline 904 & NGC 7591 & Sbc & B & 0.46 & 50 & 1 & 11 & 10.64 \\
\hline 924 & NGC 7716 & $\mathrm{Sb}$ & A & 0.19 & 50 & $\ldots$ & $\ldots$ & 10.20 \\
\hline 929 & UGC 12810 & Sbc & B & 0.61 & 50 & $\ldots$ & $\ldots$ & 10.61 \\
\hline 938 & NGC 5947 & Sbc & B & 0.15 & 50 & 26 & 11 & 10.23 \\
\hline
\end{tabular}


J. K. Barrera-Ballesteros et al.: Kinematic alignment of non-interacting CALIFA galaxies

Table B.2. Stellar kinematic properties of the non-interacting sample selected for this study included in the CALIFA survey.

\begin{tabular}{|c|c|c|c|c|c|c|c|c|c|c|}
\hline \multirow{2}{*}{$\begin{array}{l}\text { id } \\
\text { (1) }\end{array}$} & \multirow{2}{*}{$\begin{array}{c}\Delta \alpha \\
(\operatorname{arcsec}) \\
(2)\end{array}$} & \multirow{2}{*}{$\begin{array}{c}\Delta \delta \\
(\operatorname{arcsec}) \\
(3)\end{array}$} & \multirow{2}{*}{$\begin{array}{c}V_{\text {sys }} \\
\left(\mathrm{km} \mathrm{s}^{-1}\right) \\
(4)\end{array}$} & \multirow{2}{*}{$\begin{array}{c}r_{\max } \\
(\operatorname{arcsec}) \\
(5)\end{array}$} & \multirow{2}{*}{$\begin{array}{c}r_{\min } \\
(\operatorname{arcsec}) \\
(6)\end{array}$} & \multirow{2}{*}{$\begin{array}{c}\mathrm{PA}_{\operatorname{morph}}\left(r_{\max }\right) \\
\left({ }^{\circ}\right) \\
(7)\end{array}$} & \multicolumn{2}{|c|}{ PA approaching } & \multicolumn{2}{|c|}{ PA receding } \\
\hline & & & & & & & $\begin{array}{l}\mathrm{PA}_{\text {kin }} \\
\left({ }^{\circ}\right) \\
(8)\end{array}$ & $\begin{array}{c}\delta \mathrm{PA}_{\text {kin }} \\
\left({ }^{\circ}\right) \\
(9)\end{array}$ & $\begin{array}{l}\mathrm{PA}_{\text {kin }} \\
\left({ }^{\circ}\right) \\
(10)\end{array}$ & $\begin{array}{c}\delta \mathrm{PA}_{\text {kin }} \\
\left({ }^{\circ}\right) \\
(11)\end{array}$ \\
\hline 1 & $0.6 \pm 0.6$ & $1.7 \pm 0.5$ & $4963 \pm 9$ & 22 & - & $3.7 \pm 0.4$ & $6.0 \pm 3.3$ & $7.1 \pm 2.2$ & $8.2 \pm 3.2$ & $8.0 \pm 1.8$ \\
\hline 2 & $\ldots$ & $\ldots$ & $7209 \pm 1$ & 22 & - & $45.2 \pm 0.9$ & $49.9 \pm 2.7$ & $10.5 \pm 2.7$ & $45.0 \pm 0.2$ & $15.2 \pm 2.7$ \\
\hline 7 & & & $6238 \pm 1$ & 20 & - & $17.4 \pm 0.2$ & $22.7 \pm 2.8$ & $10.7 \pm 2.3$ & $18.8 \pm 1.6$ & $7.3 \pm 1.8$ \\
\hline 8 & $-0.6 \pm 0.5$ & $-1.1 \pm 0.6$ & $4515 \pm 9$ & 20 & 7 & $106.0 \pm 1.1$ & $114.6 \pm 4.2$ & $15.0 \pm 4.9$ & $118.2 \pm 6.2$ & $20.1 \pm 3.7$ \\
\hline 10 & $\ldots$ & $\ldots$ & $5939 \pm 1$ & 25 & 10 & $179.3 \pm 0.6$ & $19.4 \pm 2.7$ & $14.4 \pm 2.1$ & $12.0 \pm 2.3$ & $12.7 \pm 2.4$ \\
\hline 23 & $0.4 \pm 0.5$ & $-1.0 \pm 0.6$ & $3857 \pm 1$ & 20 & 10 & $126.0 \pm 0.7$ & $88.8 \pm 7.2$ & $29.2 \pm 7.6$ & $92.0 \pm 4.9$ & $37.9 \pm 10.2$ \\
\hline 25 & $0.4 \pm 0.5$ & $-0.7 \pm 0.4$ & $5204 \pm 6$ & 30 & 7 & $167.1 \pm 1.0$ & $168.1 \pm 4.0$ & $18.7 \pm 3.0$ & $172.0 \pm 3.8$ & $18.0 \pm 3.4$ \\
\hline 28 & $0.2 \pm 0.5$ & $0.2 \pm 0.7$ & $4500 \pm 12$ & 12 & 5 & $60.4 \pm 0.8$ & $51.3 \pm 5.4$ & $12.7 \pm 4.7$ & $40.2 \pm 6.3$ & $18.0 \pm 4.9$ \\
\hline 30 & & & $4142 \pm 1$ & 20 & 7 & $177.3 \pm 0.8$ & $17.0 \pm 5.8$ & $24.3 \pm 5.8$ & $165.3 \pm 5.2$ & $30.6 \pm 7.4$ \\
\hline 31 & $1.9 \pm 0.7$ & $-1.1 \pm 0.8$ & $4391 \pm 6$ & 30 & 5 & $82.2 \pm 4.9$ & $66.5 \pm 7.3$ & $37.6 \pm 5.5$ & $76.4 \pm 6.7$ & $30.3 \pm 9.4$ \\
\hline 33 & $-1.1 \pm 0.7$ & $1.2 \pm 0.8$ & $5183 \pm 12$ & 20 & 7 & $93.7 \pm 1.5$ & $91.0 \pm 6.3$ & $25.7 \pm 5.6$ & $94.4 \pm 4.8$ & $25.8 \pm 7.6$ \\
\hline 41 & $1.3 \pm 0.5$ & $-1.4 \pm 0.6$ & $5524 \pm 9$ & 15 & - & $53.9 \pm 0.2$ & $58.6 \pm 7.1$ & $37.8 \pm 9.9$ & $60.3 \pm 5.1$ & $25.4 \pm 7.5$ \\
\hline 43 & & & $4839 \pm 9$ & 12 & 7 & $156.4 \pm 1.0$ & $19.0 \pm 9.7$ & $20.4 \pm 8.1$ & $31.8 \pm 7.2$ & $19.4 \pm 6.4$ \\
\hline 53 & $-0.8 \pm 0.5$ & $0.1 \pm 0.6$ & $6294 \pm 12$ & 20 & - & $152.2 \pm 0.5$ & $152.6 \pm 4.7$ & $22.1 \pm 4.7$ & $155.1 \pm 3.8$ & $12.4 \pm 2.9$ \\
\hline 108 & & & $5224 \pm 1$ & 20 & 5 & $96.5 \pm 1.2$ & $92.9 \pm 3.0$ & $13.0 \pm 5.0$ & $101.4 \pm 1.8$ & $13.7 \pm 4.2$ \\
\hline 116 & $-0.8 \pm 0.4$ & $0.1 \pm 0.8$ & $7676 \pm 21$ & 10 & - & $172.4 \pm 1.4$ & $164.5 \pm 6.3$ & $20.8 \pm 7.8$ & $171.7 \pm 7.2$ & $16.7 \pm 7.1$ \\
\hline 131 & $0.2 \pm 0.5$ & $-1.8 \pm 0.6$ & $3689 \pm 10$ & 18 & - & $128.0 \pm 0.4$ & $123.4 \pm 3.6$ & $11.7 \pm 2.6$ & $129.2 \pm 2.8$ & $11.0 \pm 2.7$ \\
\hline 147 & & & $3549 \pm 0$ & 25 & 7 & $141.7 \pm 1.9$ & $117.0 \pm 5.7$ & $25.5 \pm 3.5$ & $122.1 \pm 3.3$ & $21.1 \pm 5.0$ \\
\hline 149 & $-0.3 \pm 0.5$ & $-0.0 \pm 0.4$ & $4396 \pm 12$ & 20 & 5 & $3.9 \pm 0.8$ & $8.5 \pm 4.3$ & $13.0 \pm 3.1$ & $7.2 \pm 4.0$ & $10.0 \pm 3.7$ \\
\hline 151 & $1.4 \pm 0.5$ & $0.5 \pm 0.5$ & $4662 \pm 9$ & 29 & - & $37.0 \pm 0.3$ & $32.4 \pm 2.5$ & $9.1 \pm 1.8$ & $41.7 \pm 2.3$ & $7.9 \pm 1.4$ \\
\hline 152 & $0.6 \pm 0.6$ & $1.3 \pm 0.7$ & $3881 \pm 9$ & 20 & - & $121.9 \pm 0.5$ & $116.9 \pm 5.9$ & $21.7 \pm 5.3$ & $125.2 \pm 10.0$ & $41.2 \pm 14.8$ \\
\hline 153 & $-3.1 \pm 0.7$ & $-1.6 \pm 0.8$ & $7984 \pm 18$ & 20 & - & $134.9 \pm 0.3$ & $137.7 \pm 2.2$ & $11.2 \pm 3.0$ & $130.2 \pm 2.4$ & $9.8 \pm 3.8$ \\
\hline 273 & $1.3 \pm 0.7$ & $1.9 \pm 0.8$ & $4326 \pm 11$ & 33 & - & $162.9 \pm 0.2$ & $162.6 \pm 2.7$ & $12.6 \pm 2.1$ & $164.5 \pm 2.5$ & $12.1 \pm 2.3$ \\
\hline 275 & $-1.0 \pm 0.4$ & $-0.3 \pm 0.4$ & $2169 \pm 5$ & 27 & 5 & $82.1 \pm 0.6$ & $79.6 \pm 4.6$ & $13.9 \pm 2.6$ & $80.2 \pm 4.9$ & $20.6 \pm 4.6$ \\
\hline 277 & $-0.3 \pm 0.4$ & $-0.8 \pm 0.5$ & $3690 \pm 6$ & 25 & 9 & $22.3 \pm 0.7$ & $19.7 \pm 5.9$ & $22.4 \pm 3.4$ & $19.6 \pm 6.4$ & $19.4 \pm 2.9$ \\
\hline 307 & $\ldots$ & .. & $8344 \pm 16$ & 15 & - & $95.2 \pm 0.5$ & $101.8 \pm 6.3$ & $23.9 \pm 13.3$ & $95.5 \pm 5.6$ & $22.7 \pm 7.2$ \\
\hline 364 & $\ldots$ & $\ldots$ & $6474 \pm 1$ & 25 & - & $99.8 \pm 0.4$ & $102.6 \pm 1.4$ & $6.2 \pm 0.8$ & $101.8 \pm 0.8$ & $5.9 \pm 1.0$ \\
\hline 386 & $0.5 \pm 0.6$ & $0.4 \pm 0.5$ & $6297 \pm 10$ & 17 & - & $48.9 \pm 0.9$ & $44.9 \pm 3.9$ & $14.0 \pm 3.2$ & $58.1 \pm 4.7$ & $17.4 \pm 3.9$ \\
\hline 414 & & 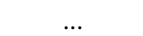 & $2521 \pm 0$ & 25 & 7 & $107.9 \pm 3.2$ & $135.6 \pm 12.8$ & $36.8 \pm 7.8$ & $141.5 \pm 11.0$ & $38.7 \pm 6.4$ \\
\hline 436 & $0.3 \pm 0.7$ & $0.5 \pm 0.7$ & $3106 \pm 9$ & 20 & 5 & $24.3 \pm 1.1$ & $145.1 \pm 39.0$ & $31.2 \pm 8.0$ & $173.2 \pm 5.9$ & $37.8 \pm 11.5$ \\
\hline 489 & $-0.2 \pm 0.4$ & $-0.9 \pm 0.7$ & $3392 \pm 5$ & 25 & 10 & $104.1 \pm 1.0$ & $102.4 \pm 3.7$ & $16.6 \pm 3.9$ & $106.6 \pm 3.8$ & $16.1 \pm 3.4$ \\
\hline 515 & $\ldots$ & $\ldots$ & $3868 \pm 1$ & 25 & 10 & $0.2 \pm 1.1$ & $159.5 \pm 2.3$ & $12.1 \pm 3.0$ & $171.8 \pm 5.4$ & $21.0 \pm 3.1$ \\
\hline 518 & $\ldots$ & $\ldots$ & $2712 \pm 0$ & 30 & 5 & $88.5 \pm 1.0$ & $99.2 \pm 4.4$ & $24.2 \pm 3.5$ & $93.0 \pm 3.5$ & $23.4 \pm 4.4$ \\
\hline 580 & $\ldots$ & $\ldots$ & $4082 \pm 1$ & 23 & 5 & $43.3 \pm 0.5$ & $40.6 \pm 4.4$ & $21.4 \pm 3.9$ & $36.1 \pm 5.1$ & $19.8 \pm 3.7$ \\
\hline 602 & $-0.4 \pm 0.2$ & $-1.0 \pm 0.3$ & $4737 \pm 5$ & 15 & - & $75.2 \pm 0.8$ & $72.9 \pm 3.2$ & $11.5 \pm 3.0$ & $82.4 \pm 4.4$ & $10.9 \pm 4.7$ \\
\hline 610 & $1.4 \pm 0.8$ & $-1.2 \pm 1.0$ & $7125 \pm 19$ & 20 & - & $39.4 \pm 0.3$ & $40.2 \pm 3.2$ & $16.7 \pm 5.0$ & $38.7 \pm 3.6$ & $15.1 \pm 4.5$ \\
\hline 624 & $0.5 \pm 0.4$ & $0.5 \pm 0.3$ & $7255 \pm 7$ & 25 & 6 & $116.4 \pm 1.5$ & $92.9 \pm 3.5$ & $18.3 \pm 2.8$ & $85.2 \pm 4.8$ & $16.8 \pm 3.2$ \\
\hline 630 & $\ldots$ & $\ldots$ & $1768 \pm 1$ & 20 & 9 & $146.4 \pm 0.7$ & $157.6 \pm 8.0$ & $35.3 \pm 8.0$ & $170.1 \pm 7.6$ & $33.3 \pm 6.5$ \\
\hline 664 & $\ldots$ & $\ldots$ & $3220 \pm 0$ & 25 & - & $118.7 \pm 0.2$ & $109.4 \pm 2.6$ & $13.0 \pm 2.0$ & $111.5 \pm 2.9$ & $18.7 \pm 2.7$ \\
\hline 684 & $-1.5 \pm 0.3$ & $-0.2 \pm 0.3$ & $5331 \pm 6$ & 27 & 7 & $141.2 \pm 2.1$ & $119.9 \pm 2.3$ & $14.1 \pm 2.5$ & $107.5 \pm 4.6$ & $13.7 \pm 2.6$ \\
\hline 714 & $0.4 \pm 0.7$ & $-0.4 \pm 0.6$ & $7793 \pm 18$ & 20 & - & $16.7 \pm 0.5$ & $12.7 \pm 5.0$ & $13.0 \pm 2.9$ & $13.0 \pm 4.3$ & $16.7 \pm 4.1$ \\
\hline 715 & $-0.7 \pm 0.8$ & $0.0 \pm 0.7$ & $1885 \pm 9$ & 30 & 7 & $66.5 \pm 0.4$ & $68.7 \pm 3.9$ & $17.0 \pm 3.4$ & $62.4 \pm 5.0$ & $21.7 \pm 3.6$ \\
\hline 743 & $-0.1 \pm 0.6$ & $1.3 \pm 0.7$ & $3868 \pm 8$ & 25 & 5 & $85.9 \pm 0.5$ & $92.1 \pm 7.5$ & $32.4 \pm 5.2$ & $81.0 \pm 6.4$ & $25.7 \pm 6.5$ \\
\hline 748 & $0.3 \pm 0.7$ & $-0.4 \pm 0.4$ & $2355 \pm 4$ & 25 & 10 & $192.5 \pm 0.5$ & $17.8 \pm 6.2$ & $24.0 \pm 4.8$ & $17.6 \pm 4.1$ & $28.3 \pm 6.3$ \\
\hline 753 & $-0.3 \pm 0.4$ & $-0.5 \pm 0.5$ & $3175 \pm 4$ & 25 & 7 & $56.1 \pm 0.6$ & $57.4 \pm 3.0$ & $15.6 \pm 2.8$ & $57.0 \pm 3.2$ & $10.1 \pm 1.8$ \\
\hline 764 & $1.0 \pm 0.7$ & $-1.1 \pm 0.8$ & $7704 \pm 11$ & 20 & 7 & $128.4 \pm 0.7$ & $121.3 \pm 4.0$ & $16.0 \pm 4.8$ & $136.1 \pm 7.8$ & $22.5 \pm 4.2$ \\
\hline 768 & $-1.0 \pm 0.5$ & $-0.2 \pm 0.6$ & $3735 \pm 8$ & 15 & 7 & $42.4 \pm 0.9$ & $50.0 \pm 20.6$ & $37.6 \pm 12.1$ & $41.7 \pm 13.8$ & $41.7 \pm 7.4$ \\
\hline 771 & $-1.1 \pm 0.8$ & $-1.8 \pm 0.9$ & $3762 \pm 9$ & 20 & 7 & $98.7 \pm 0.9$ & $29.5 \pm 6.6$ & $29.5 \pm 7.6$ & $46.5 \pm 9.7$ & $39.5 \pm 14.3$ \\
\hline 777 & $0.5 \pm 0.4$ & $-1.0 \pm 0.4$ & $4836 \pm 9$ & 30 & 10 & $36.1 \pm 0.4$ & $35.7 \pm 2.0$ & $10.1 \pm 1.6$ & $38.4 \pm 3.1$ & $12.3 \pm 1.9$ \\
\hline 779 & $-1.8 \pm 0.5$ & $-1.1 \pm 0.6$ & $5527 \pm 8$ & 23 & - & $121.8 \pm 0.3$ & $129.4 \pm 4.4$ & $22.1 \pm 5.0$ & $117.9 \pm 3.7$ & $16.7 \pm 5.7$ \\
\hline 782 & $0.9 \pm 0.3$ & $0.9 \pm 0.3$ & $7803 \pm 10$ & 12 & - & $152.9 \pm 0.5$ & $147.6 \pm 3.0$ & $10.3 \pm 3.3$ & $152.9 \pm 3.6$ & $21.0 \pm 8.2$ \\
\hline
\end{tabular}

Notes. (1) CALIFA identifier. (2) and (3) Location of the kinematic centre. The value listed is the gradient peak (GP) with respect to the optical nucleus $(\mathrm{ON})$ if not, $\mathrm{ON}$ is used as kinematic centre (see Sect. 3 for details). (4) Systemic velocity derived from integrated the velocities in a 2.7" aperture centred in the kinematic centre. (5) Radius used to average the polar coordinates of the positions from the lines of nodes (see Sect. 3 for details). (6) Radius used to average the polar coordinates of the positions from the zero-velocity line (see Sect. 3 for details). (7) Morphological PA inferred by fitting an elipse to an isophote at radius $r_{\max }$ in the $r$-band SDSS image. (8) Kinematic position angle at radius $r_{\max }$ for the approaching side (see Sect. 3 for details). (9) Standard deviation of the kinematic position angle of the approaching side (see Sect. 3 for details). (10) Kinematic position angle at radius $r_{\max }$ for the receding side. (11) Standard deviation of the kinematic position angle of the receding side. 
A\&A 568, A70 (2014)

Table B.2. -continued.

\begin{tabular}{|c|c|c|c|c|c|c|c|c|c|c|}
\hline \multirow{2}{*}{$\begin{array}{l}\text { id } \\
\text { (1) } \\
\end{array}$} & \multirow{2}{*}{$\begin{array}{c}\Delta \alpha \\
(\operatorname{arcsec}) \\
(2) \\
\end{array}$} & \multirow{2}{*}{$\begin{array}{c}\Delta \delta \\
(\operatorname{arcsec}) \\
(3) \\
\end{array}$} & \multirow{2}{*}{$\begin{array}{c}V_{\text {sys }} \\
\left(\mathrm{km} \mathrm{s}^{-1}\right) \\
(4)\end{array}$} & \multirow{2}{*}{$\begin{array}{c}r_{\max } \\
(\operatorname{arcsec}) \\
(5) \\
\end{array}$} & \multirow{2}{*}{$\begin{array}{c}r_{\min } \\
(\operatorname{arcsec}) \\
(6) \\
\end{array}$} & \multirow{2}{*}{$\begin{array}{c}\mathrm{PA}_{\operatorname{morph}}\left(r_{\max }\right) \\
\left({ }^{\circ}\right) \\
(7) \\
\end{array}$} & \multicolumn{2}{|c|}{ PA approaching } & \multicolumn{2}{|c|}{ PA receding } \\
\hline & & & & & & & $\begin{array}{c}\mathrm{PA}_{\mathrm{kin}} \\
\left({ }^{\circ}\right) \\
(8) \\
\end{array}$ & $\begin{array}{c}\delta \mathrm{PA}_{\mathrm{kin}} \\
\left({ }^{\circ}\right) \\
(9) \\
\end{array}$ & $\begin{array}{c}\mathrm{PA}_{\mathrm{kin}} \\
\left({ }^{\circ}\right) \\
(10) \\
\end{array}$ & $\begin{array}{c}\delta \mathrm{PA}_{\text {kin }} \\
\left({ }^{\circ}\right) \\
(11) \\
\end{array}$ \\
\hline 790 & & & $4677 \pm 1$ & 12 & 5 & $143.6 \pm 1.3$ & $142.4 \pm 5.6$ & $16.1 \pm 4.3$ & $144.5 \pm 5.9$ & $12.4 \pm 4.5$ \\
\hline 791 & $1.2 \pm 0.3$ & $0.4 \pm 0.3$ & $3316 \pm 5$ & 30 & - & $152.3 \pm 0.3$ & $149.7 \pm 1.2$ & $3.2 \pm 0.7$ & $154.5 \pm 1.3$ & $3.7 \pm 0.6$ \\
\hline 804 & $-2.2 \pm 0.5$ & $-0.5 \pm 0.5$ & $3388 \pm 7$ & 15 & - & $126.8 \pm 0.5$ & $130.5 \pm 4.4$ & $19.3 \pm 4.8$ & $127.7 \pm 4.7$ & $18.6 \pm 5.8$ \\
\hline 810 & $-0.3 \pm 0.5$ & $-0.1 \pm 0.5$ & $4117 \pm 9$ & 25 & - & $15.3 \pm 0.5$ & $8.6 \pm 3.0$ & $10.0 \pm 2.4$ & $19.6 \pm 3.1$ & $16.6 \pm 3.0$ \\
\hline 813 & $\ldots$ & $\ldots$ & $3836 \pm 1$ & 28 & 7 & $113.1 \pm 8.8$ & $101.1 \pm 5.5$ & $33.8 \pm 5.3$ & $90.0 \pm 4.4$ & $32.4 \pm 6.1$ \\
\hline 823 & $\ldots$ & $\ldots$ & $2838 \pm 1$ & 25 & 7 & $159.2 \pm 0.6$ & $152.2 \pm 4.5$ & $31.4 \pm 6.5$ & $168.5 \pm 7.8$ & $26.0 \pm 4.3$ \\
\hline 824 & $-0.4 \pm 0.5$ & $-1.7 \pm 0.6$ & $4730 \pm 14$ & 27 & - & $159.8 \pm 0.2$ & $161.6 \pm 3.0$ & $10.5 \pm 1.8$ & $152.7 \pm 4.0$ & $13.6 \pm 2.2$ \\
\hline 826 & $-0.4 \pm 0.5$ & $-1.2 \pm 0.5$ & $5070 \pm 9$ & 25 & - & $127.9 \pm 0.3$ & $133.4 \pm 2.3$ & $10.3 \pm 2.2$ & $122.5 \pm 2.2$ & $7.5 \pm 1.3$ \\
\hline 831 & $-0.3 \pm 0.8$ & $0.4 \pm 0.7$ & $4957 \pm 12$ & 15 & - & $123.4 \pm 0.6$ & $123.8 \pm 9.4$ & $30.1 \pm 9.9$ & $133.8 \pm 7.1$ & $28.2 \pm 8.4$ \\
\hline 834 & & & $8694 \pm 1$ & 15 & - & $114.2 \pm 0.5$ & $106.3 \pm 1.9$ & $8.9 \pm 2.6$ & $95.0 \pm 5.8$ & $22.0 \pm 3.4$ \\
\hline 836 & $0.6 \pm 0.8$ & $0.8 \pm 0.8$ & $2424 \pm 4$ & 29 & - & $148.9 \pm 0.9$ & $140.5 \pm 5.3$ & $28.1 \pm 7.3$ & $133.4 \pm 6.1$ & $31.1 \pm 5.0$ \\
\hline 842 & $0.3 \pm 0.5$ & $-2.2 \pm 0.5$ & $2970 \pm 6$ & 30 & 7 & $54.7 \pm 0.4$ & $66.9 \pm 5.6$ & $41.6 \pm 4.5$ & $70.4 \pm 5.3$ & $32.0 \pm 4.6$ \\
\hline 853 & $\ldots$ & $\ldots$ & $3788 \pm 1$ & 30 & 10 & $53.0 \pm 5.0$ & $53.6 \pm 2.9$ & $10.8 \pm 2.1$ & $49.5 \pm 2.2$ & $5.7 \pm 1.4$ \\
\hline 854 & $\ldots$ & $\ldots$ & $8625 \pm 1$ & 20 & - & $90.9 \pm 0.4$ & $90.0 \pm 4.3$ & $8.8 \pm 3.0$ & $90.0 \pm 4.5$ & $12.3 \pm 2.9$ \\
\hline 856 & $\ldots$ & $\ldots$ & $4696 \pm 1$ & 22 & 5 & $91.6 \pm 0.7$ & $90.0 \pm 4.3$ & $24.7 \pm 7.1$ & $93.7 \pm 3.7$ & $20.2 \pm 3.9$ \\
\hline 857 & $\ldots$ & $\ldots$ & $8453 \pm 1$ & 15 & - & $36.1 \pm 0.4$ & $47.8 \pm 3.9$ & $12.4 \pm 3.5$ & $45.0 \pm 0.2$ & $11.9 \pm 2.8$ \\
\hline 862 & $\ldots$ & $\ldots$ & $6704 \pm 1$ & 25 & - & $32.1 \pm 0.4$ & $30.2 \pm 3.4$ & $13.2 \pm 2.1$ & $31.7 \pm 2.2$ & $10.1 \pm 1.9$ \\
\hline 869 & $-0.6 \pm 0.5$ & $0.9 \pm 0.5$ & $6157 \pm 7$ & 22 & 7 & $121.8 \pm 1.8$ & $130.8 \pm 4.2$ & $25.8 \pm 3.8$ & $141.4 \pm 3.9$ & $12.5 \pm 2.8$ \\
\hline 872 & $-0.2 \pm 0.7$ & $-1.3 \pm 0.7$ & $3767 \pm 11$ & 20 & 7 & $85.4 \pm 3.0$ & $64.2 \pm 6.3$ & $21.5 \pm 3.8$ & $56.3 \pm 6.9$ & $23.1 \pm 4.3$ \\
\hline 876 & $-0.6 \pm 0.8$ & $1.4 \pm 0.8$ & $5740 \pm 13$ & 20 & 5 & $106.9 \pm 1.3$ & $94.2 \pm 4.6$ & $19.6 \pm 5.0$ & $95.6 \pm 9.2$ & $29.9 \pm 5.4$ \\
\hline 886 & $-1.4 \pm 0.3$ & $-0.1 \pm 0.2$ & $4488 \pm 5$ & 27 & 7 & $12.8 \pm 0.3$ & $4.2 \pm 1.8$ & $8.0 \pm 1.6$ & $20.0 \pm 2.2$ & $9.0 \pm 2.0$ \\
\hline 887 & $0.3 \pm 0.4$ & $0.4 \pm 0.3$ & $7065 \pm 9$ & 25 & 5 & $25.2 \pm 1.4$ & $12.6 \pm 2.9$ & $9.7 \pm 2.1$ & $16.7 \pm 4.4$ & $17.6 \pm 2.5$ \\
\hline 889 & $-1.2 \pm 0.4$ & $-0.9 \pm 0.4$ & $4852 \pm 7$ & 20 & 10 & $64.4 \pm 0.7$ & $73.4 \pm 3.9$ & $9.9 \pm 2.1$ & $58.6 \pm 2.9$ & $14.8 \pm 3.1$ \\
\hline 890 & $0.4 \pm 0.5$ & $-0.1 \pm 0.4$ & $6541 \pm 10$ & 15 & - & $142.6 \pm 0.5$ & $157.5 \pm 4.2$ & $11.0 \pm 2.6$ & $162.8 \pm 5.4$ & $14.1 \pm 2.8$ \\
\hline 896 & $0.3 \pm 0.5$ & $1.7 \pm 0.6$ & $7439 \pm 11$ & 18 & - & $19.6 \pm 0.5$ & $18.7 \pm 6.9$ & $20.0 \pm 5.5$ & $29.6 \pm 5.6$ & $23.0 \pm 4.6$ \\
\hline 904 & $-0.5 \pm 0.5$ & $-0.0 \pm 0.5$ & $4913 \pm 13$ & 30 & 6 & $148.2 \pm 1.1$ & $149.8 \pm 2.5$ & $14.2 \pm 2.6$ & $145.1 \pm 2.6$ & $12.7 \pm 2.6$ \\
\hline 924 & $-1.0 \pm 0.0$ & $-1.0 \pm 0.0$ & $2571 \pm 1$ & 22 & 7 & $24.6 \pm 0.8$ & $37.4 \pm 1.8$ & $12.0 \pm 1.7$ & $51.4 \pm 3.2$ & $14.7 \pm 3.7$ \\
\hline 929 & $1.4 \pm 0.8$ & $0.1 \pm 0.7$ & $7977 \pm 17$ & 20 & - & $43.4 \pm 0.8$ & $54.9 \pm 4.6$ & $16.8 \pm 3.6$ & $58.2 \pm 4.0$ & $15.5 \pm 3.6$ \\
\hline 938 & $1.0 \pm 0.6$ & $1.7 \pm 0.5$ & $5896 \pm 4$ & 15 & 7 & $51.1 \pm 5.9$ & $75.3 \pm 10.7$ & $34.0 \pm 6.3$ & $73.2 \pm 8.1$ & $26.1 \pm 5.4$ \\
\hline
\end{tabular}


J. K. Barrera-Ballesteros et al.: Kinematic alignment of non-interacting CALIFA galaxies

Table B.3. Ionized gas kinematic properties of the non-interacting sample selected for this study included in the CALIFA survey.

\begin{tabular}{|c|c|c|c|c|c|c|c|c|c|c|}
\hline \multirow{2}{*}{$\begin{array}{l}\text { id } \\
\text { (1) }\end{array}$} & \multirow{2}{*}{$\begin{array}{c}\Delta \alpha \\
(\operatorname{arcsec}) \\
(2)\end{array}$} & \multirow{2}{*}{$\begin{array}{c}\Delta \delta \\
(\operatorname{arcsec}) \\
(3)\end{array}$} & \multirow{2}{*}{$\begin{array}{c}V_{\text {sys }} \\
\left(\mathrm{km} \mathrm{s}^{-1}\right) \\
(4)\end{array}$} & \multirow{2}{*}{$\begin{array}{c}r_{\max } \\
(\operatorname{arcsec}) \\
(5)\end{array}$} & \multirow{2}{*}{$\begin{array}{c}r_{\min } \\
(\operatorname{arcsec}) \\
(6)\end{array}$} & \multirow{2}{*}{$\begin{array}{c}\mathrm{PA}_{\operatorname{morph}}\left(r_{\max }\right) \\
\left({ }^{\circ}\right) \\
(7)\end{array}$} & \multicolumn{2}{|c|}{ PA approaching } & \multicolumn{2}{|c|}{ PA receding } \\
\hline & & & & & & & $\begin{array}{l}\mathrm{PA}_{\mathrm{kin}} \\
\left({ }^{\circ}\right) \\
(8)\end{array}$ & $\begin{array}{c}\delta \mathrm{PA}_{\mathrm{kin}} \\
\left({ }^{\circ}\right) \\
(9)\end{array}$ & $\begin{array}{l}\mathrm{PA}_{\mathrm{kin}} \\
\left({ }^{\circ}\right) \\
(10)\end{array}$ & $\begin{array}{c}\delta \mathrm{PA}_{\mathrm{kin}} \\
\left({ }^{\circ}\right) \\
(11)\end{array}$ \\
\hline 1 & $\ldots$ & $\ldots$ & $4990 \pm 2$ & 22 & $\ldots$ & $3.7 \pm 0.4$ & $4.5 \pm 1.2$ & $3.1 \pm 1.0$ & $3.5 \pm 1.6$ & $3.5 \pm 1.3$ \\
\hline 2 & $0.2 \pm 0.1$ & $-2.3 \pm 0.1$ & $7247 \pm 1$ & 22 & $\begin{array}{l}\cdots \\
\cdots\end{array}$ & $45.2 \pm 0.9$ & $55.0 \pm 1.4$ & $6.1 \pm 0.7$ & $44.6 \pm 1.0$ & $6.5 \pm 1.5$ \\
\hline 7 & $-1.4 \pm 0.3$ & $-0.5 \pm 0.4$ & $6299 \pm 18$ & 20 & $\ldots$ & $17.4 \pm 0.2$ & $27.8 \pm 2.7$ & $8.6 \pm 2.1$ & $10.3 \pm 2.4$ & $6.8 \pm 1.7$ \\
\hline 8 & $0.1 \pm 0.1$ & $0.9 \pm 0.1$ & $4534 \pm 0$ & 20 & $\dddot{7}$ & $106.0 \pm 1.1$ & $116.2 \pm 2.1$ & $7.4 \pm 1.0$ & $115.7 \pm 1.7$ & $7.3 \pm 1.0$ \\
\hline 10 & $0.1 \pm 0.2$ & $-0.7 \pm 0.3$ & $5974 \pm 8$ & 25 & 10 & $179.3 \pm 0.6$ & $16.4 \pm 1.4$ & $8.3 \pm 1.5$ & $18.9 \pm 2.3$ & $13.9 \pm 2.5$ \\
\hline 23 & $-0.5 \pm 0.6$ & $-0.9 \pm 0.6$ & $3859 \pm 6$ & 20 & 10 & $126.0 \pm 0.7$ & $69.9 \pm 16.1$ & $91.4 \pm 9.2$ & $108.2 \pm 33.0$ & $91.4 \pm 21.9$ \\
\hline 25 & $-1.8 \pm 0.5$ & $-1.2 \pm 0.6$ & $5239 \pm 10$ & 30 & 7 & $167.1 \pm 1.0$ & $174.6 \pm 2.4$ & $14.5 \pm 3.2$ & $165.6 \pm 1.8$ & $19.8 \pm 2.7$ \\
\hline 28 & $-0.4 \pm 0.2$ & $0.0 \pm 0.3$ & $4486 \pm 4$ & 12 & 5 & $60.4 \pm 0.8$ & $56.2 \pm 2.3$ & $11.0 \pm 2.6$ & $47.3 \pm 2.4$ & $8.6 \pm 2.5$ \\
\hline 30 & $0.1 \pm 0.1$ & $1.4 \pm 0.1$ & $4111 \pm 2$ & 20 & 7 & $177.3 \pm 0.8$ & $5.7 \pm 1.9$ & $6.8 \pm 1.7$ & $179.6 \pm 0.6$ & $6.9 \pm 3.0$ \\
\hline 31 & $-0.8 \pm 0.2$ & $1.5 \pm 0.2$ & $4438 \pm 2$ & 30 & 5 & $82.2 \pm 4.9$ & $65.4 \pm 1.1$ & $7.2 \pm 1.3$ & $75.9 \pm 1.9$ & $10.5 \pm 2.2$ \\
\hline 33 & $-0.2 \pm 0.2$ & $0.5 \pm 0.2$ & $5213 \pm 6$ & 20 & 7 & $93.7 \pm 1.5$ & $91.5 \pm 2.1$ & $7.5 \pm 1.1$ & $87.7 \pm 1.2$ & $6.9 \pm 1.3$ \\
\hline 41 & $\ldots$ & $\ldots$ & $5547 \pm 0$ & 15 & $\ldots$ & $53.9 \pm 0.2$ & $45.9 \pm 2.2$ & $9.6 \pm 2.4$ & $60.6 \pm 2.2$ & $6.1 \pm 1.3$ \\
\hline 43 & $-0.1 \pm 0.3$ & $1.0 \pm 0.4$ & $4811 \pm 7$ & 12 & 7 & $156.4 \pm 1.0$ & $7.9 \pm 4.0$ & $12.3 \pm 3.8$ & $16.1 \pm 4.5$ & $11.5 \pm 4.3$ \\
\hline 53 & $-1.2 \pm 0.3$ & $-1.8 \pm 0.3$ & $6356 \pm 4$ & 20 & $\ldots$ & $152.2 \pm 0.5$ & $148.0 \pm 1.4$ & $4.8 \pm 1.0$ & $151.7 \pm 1.6$ & $5.8 \pm 1.2$ \\
\hline 116 & $-0.7 \pm 0.4$ & $1.4 \pm 0.4$ & $7696 \pm 12$ & 10 & $\ldots$ & $172.4 \pm 1.4$ & $159.9 \pm 4.9$ & $8.3 \pm 2.7$ & $167.3 \pm 7.0$ & $12.3 \pm 5.0$ \\
\hline 131 & $-0.1 \pm 0.5$ & $-0.4 \pm 0.5$ & $3693 \pm 7$ & 18 & $\ldots$ & $128.0 \pm 0.4$ & $130.3 \pm 3.5$ & $8.2 \pm 2.5$ & $132.9 \pm 2.9$ & $7.0 \pm 1.9$ \\
\hline 147 & $0.1 \pm 0.1$ & $2.2 \pm 0.2$ & $3568 \pm 2$ & 25 & 7 & $141.7 \pm 1.9$ & $117.7 \pm 1.4$ & $8.1 \pm 1.4$ & $116.4 \pm 4.3$ & $14.3 \pm 2.6$ \\
\hline 149 & $0.6 \pm 0.2$ & $-0.6 \pm 0.2$ & $4359 \pm 10$ & 20 & 5 & $3.9 \pm 0.8$ & $5.3 \pm 1.7$ & $4.7 \pm 0.9$ & $13.8 \pm 1.7$ & $5.9 \pm 1.5$ \\
\hline 151 & $0.7 \pm 0.3$ & $-0.3 \pm 0.2$ & $4574 \pm 18$ & 29 & $\ldots$ & $37.0 \pm 0.3$ & $36.6 \pm 1.3$ & $4.3 \pm 0.8$ & $37.3 \pm 3.0$ & $10.8 \pm 3.7$ \\
\hline 152 & $-0.1 \pm 0.3$ & $-1.1 \pm 0.3$ & $3916 \pm 4$ & 20 & $\cdots$ & $121.9 \pm 0.5$ & $122.8 \pm 1.9$ & $8.2 \pm 2.2$ & $113.9 \pm 2.2$ & $8.1 \pm 1.8$ \\
\hline 153 & $-0.6 \pm 0.4$ & $-0.7 \pm 0.3$ & $8081 \pm 10$ & 20 & $\cdots$ & $134.9 \pm 0.3$ & $135.7 \pm 2.1$ & $7.3 \pm 1.8$ & $134.0 \pm 1.9$ & $4.8 \pm 1.1$ \\
\hline 273 & $2.4 \pm 0.9$ & $-1.9 \pm 0.6$ & $4270 \pm 5$ & 33 & $\ldots$ & $162.9 \pm 0.2$ & $150.0 \pm 2.9$ & $10.7 \pm 2.1$ & $174.1 \pm 2.6$ & $6.2 \pm 1.5$ \\
\hline 275 & $1.2 \pm 0.2$ & $0.6 \pm 0.1$ & $2086 \pm 6$ & 27 & 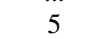 & $82.1 \pm 0.6$ & $83.2 \pm 0.9$ & $3.9 \pm 0.8$ & $82.3 \pm 1.3$ & $8.1 \pm 1.9$ \\
\hline 277 & $0.9 \pm 0.4$ & $0.4 \pm 0.6$ & $3649 \pm 6$ & 25 & 9 & $22.3 \pm 0.7$ & $12.8 \pm 2.5$ & $16.1 \pm 2.8$ & $32.5 \pm 4.0$ & $26.6 \pm 3.1$ \\
\hline 307 & $-0.2 \pm 0.3$ & $0.2 \pm 0.3$ & $8439 \pm 10$ & 15 & $\ldots$ & $95.2 \pm 0.5$ & $97.2 \pm 4.3$ & $9.1 \pm 3.4$ & $92.0 \pm 3.2$ & $6.3 \pm 2.5$ \\
\hline 364 & $0.2 \pm 0.6$ & $1.1 \pm 0.6$ & $6582 \pm 22$ & 25 & $\ldots$ & $99.8 \pm 0.4$ & $108.6 \pm 4.3$ & $14.3 \pm 2.7$ & $98.6 \pm 2.8$ & $6.4 \pm 1.7$ \\
\hline 386 & $-0.7 \pm 0.5$ & $-0.1 \pm 0.6$ & $6332 \pm 10$ & 17 & $\begin{array}{l}\cdots \\
\ldots\end{array}$ & $48.9 \pm 0.9$ & $46.6 \pm 3.4$ & $8.8 \pm 2.6$ & $44.2 \pm 2.9$ & $10.6 \pm 2.6$ \\
\hline 414 & $-0.4 \pm 0.3$ & $0.1 \pm 0.3$ & $2497 \pm 3$ & 25 & 7 & $107.9 \pm 3.2$ & $143.4 \pm 2.3$ & $13.8 \pm 2.6$ & $150.5 \pm 2.8$ & $17.1 \pm 3.7$ \\
\hline 436 & $0.1 \pm 0.3$ & $-1.5 \pm 0.3$ & $3158 \pm 6$ & 20 & 5 & $24.3 \pm 1.1$ & $171.5 \pm 3.1$ & $12.1 \pm 4.7$ & $173.7 \pm 2.7$ & $7.7 \pm 1.9$ \\
\hline 489 & $0.8 \pm 0.1$ & $-0.6 \pm 0.2$ & $3409 \pm 1$ & 25 & 10 & $104.1 \pm 1.0$ & $100.8 \pm 1.5$ & $8.8 \pm 1.3$ & $104.2 \pm 2.0$ & $8.1 \pm 1.2$ \\
\hline 515 & $0.1 \pm 0.4$ & $0.1 \pm 0.3$ & $3866 \pm 7$ & 25 & 10 & $0.2 \pm 1.1$ & $166.6 \pm 3.3$ & $10.5 \pm 1.9$ & $165.4 \pm 3.2$ & $10.1 \pm 1.8$ \\
\hline 610 & $0.5 \pm 0.4$ & $1.1 \pm 0.5$ & $7223 \pm 12$ & 20 & $\cdots$ & $39.4 \pm 0.3$ & $39.9 \pm 2.0$ & $5.6 \pm 1.3$ & $47.0 \pm 2.6$ & $7.1 \pm 1.5$ \\
\hline 624 & $0.9 \pm 0.6$ & $-0.3 \pm 0.4$ & $7309 \pm 7$ & 25 & 6 & $116.4 \pm 1.5$ & $87.4 \pm 4.6$ & $25.9 \pm 5.1$ & $78.1 \pm 7.9$ & $38.4 \pm 4.2$ \\
\hline 630 & $-0.1 \pm 0.4$ & $0.0 \pm 0.5$ & $1727 \pm 7$ & 20 & 9 & $146.4 \pm 0.7$ & $159.5 \pm 4.0$ & $18.5 \pm 4.4$ & $163.9 \pm 3.7$ & $18.1 \pm 4.3$ \\
\hline 664 & ... & $\ldots$ & $3204 \pm 5$ & 25 & $\ldots$ & $118.7 \pm 0.2$ & $113.3 \pm 3.2$ & $19.8 \pm 3.3$ & $103.6 \pm 3.2$ & $13.6 \pm 3.5$ \\
\hline 684 & $0.4 \pm 0.4$ & $1.2 \pm 0.4$ & $5439 \pm 10$ & 27 & 7 & $141.2 \pm 2.1$ & $109.5 \pm 3.8$ & $15.3 \pm 4.5$ & $111.3 \pm 4.2$ & $15.0 \pm 2.0$ \\
\hline 714 & $-0.2 \pm 0.2$ & $0.4 \pm 0.2$ & $7831 \pm 10$ & 20 & $\ldots$ & $16.7 \pm 0.5$ & $12.1 \pm 2.4$ & $5.6 \pm 1.3$ & $13.5 \pm 1.9$ & $4.3 \pm 1.0$ \\
\hline 715 & $-1.0 \pm 0.1$ & $0.7 \pm 0.0$ & $1884 \pm 1$ & 30 & 7 & $66.5 \pm 0.4$ & $64.9 \pm 0.5$ & $4.3 \pm 0.6$ & $66.7 \pm 0.8$ & $7.3 \pm 1.6$ \\
\hline 743 & $0.3 \pm 0.2$ & $-0.3 \pm 0.1$ & $3865 \pm 5$ & 25 & 5 & $85.9 \pm 0.5$ & $83.0 \pm 1.6$ & $6.0 \pm 0.9$ & $84.9 \pm 1.3$ & $8.5 \pm 1.0$ \\
\hline 748 & $-0.9 \pm 0.1$ & $-2.7 \pm 0.1$ & $2350 \pm 2$ & 25 & 10 & $192.5 \pm 0.5$ & $10.7 \pm 0.7$ & $6.1 \pm 1.0$ & $21.4 \pm 1.4$ & $10.3 \pm 1.4$ \\
\hline 753 & $0.8 \pm 0.1$ & $-0.6 \pm 0.1$ & $3222 \pm 4$ & 25 & 7 & $56.1 \pm 0.6$ & $58.4 \pm 0.7$ & $8.4 \pm 0.7$ & $55.7 \pm 0.6$ & $4.0 \pm 0.4$ \\
\hline 764 & $-0.9 \pm 0.3$ & $0.7 \pm 0.2$ & $7726 \pm 12$ & 20 & 7 & $128.4 \pm 0.7$ & $129.4 \pm 2.8$ & $10.2 \pm 2.4$ & $128.4 \pm 2.3$ & $12.4 \pm 3.9$ \\
\hline 768 & $0.4 \pm 0.2$ & $-0.0 \pm 0.2$ & $3765 \pm 3$ & 15 & 7 & $42.4 \pm 0.9$ & $36.8 \pm 1.5$ & $8.5 \pm 1.8$ & $53.7 \pm 1.8$ & $7.7 \pm 1.8$ \\
\hline 771 & $2.2 \pm 0.3$ & $-0.5 \pm 0.4$ & $3741 \pm 9$ & 20 & 7 & $98.7 \pm 0.9$ & $40.7 \pm 3.9$ & $15.8 \pm 2.7$ & $44.3 \pm 2.8$ & $11.5 \pm 2.9$ \\
\hline 777 & $-0.5 \pm 0.2$ & $0.6 \pm 0.2$ & $4877 \pm 9$ & 30 & 10 & $36.1 \pm 0.4$ & $37.4 \pm 1.0$ & $4.7 \pm 0.9$ & $36.4 \pm 1.2$ & $6.0 \pm 1.0$ \\
\hline 779 & $-0.1 \pm 0.3$ & $-1.1 \pm 0.3$ & $5559 \pm 5$ & 23 & $\ldots$ & $121.8 \pm 0.3$ & $124.9 \pm 2.1$ & $6.5 \pm 1.3$ & $122.5 \pm 2.0$ & $8.4 \pm 1.7$ \\
\hline 782 & $0.3 \pm 0.5$ & $0.5 \pm 0.5$ & $7878 \pm 29$ & 12 & $\begin{array}{l}\cdots \\
\ldots\end{array}$ & $152.9 \pm 0.5$ & $153.0 \pm 3.6$ & $10.4 \pm 2.9$ & $149.0 \pm 4.6$ & $11.9 \pm 3.1$ \\
\hline
\end{tabular}

Notes. (1) CALIFA identifier. (2) and (3) Location of the kinematic centre. The value listed is the gradient peak (GP) with respect to the optical nucleus $(\mathrm{ON})$ if not, $\mathrm{ON}$ is used as kinematic centre (see Sect. 3 for details). (4) Systemic velocity derived from integrated the velocities in a 2.7" aperture centred on the kinematic centre. (5) Radius used to average the polar coordinates of the positions from the lines of nodes (see Sect. 3 for details). (6) Radius used to average the polar coordinates of the positions from the zero-velocity line (see Sect. 3 for details). (7) Morphological PA inferred by fitting an elipse to an isophote at radius $r_{\max }$ in the $r$-band SDSS image. (8) Kinematic position angle at radius $r_{\max }$ for the approaching side (see Sect. 3 for details). (9) Standard deviation of the kinematic position angle of the approaching side (see Sect. 3 for details). (10) Kinematic position angle at radius $r_{\max }$ for the receding side. (11) Standard deviation of the kinematic position angle of the receding side. 
A\&A 568, A70 (2014)

Table B.3. continued.

\begin{tabular}{|c|c|c|c|c|c|c|c|c|c|c|}
\hline \multirow{2}{*}{$\begin{array}{l}\text { id } \\
\text { (1) }\end{array}$} & \multirow{2}{*}{$\begin{array}{c}\Delta \alpha \\
(\operatorname{arcsec}) \\
(2)\end{array}$} & \multirow{2}{*}{$\begin{array}{c}\Delta \delta \\
(\operatorname{arcsec}) \\
(3)\end{array}$} & \multirow{2}{*}{$\begin{array}{c}V_{\text {sys }} \\
\left(\mathrm{km} \mathrm{s}^{-1}\right) \\
(4)\end{array}$} & \multirow{2}{*}{$\begin{array}{c}r_{\max } \\
(\operatorname{arcsec}) \\
(5)\end{array}$} & \multirow{2}{*}{$\begin{array}{c}r_{\min } \\
(\operatorname{arcsec}) \\
(6)\end{array}$} & \multirow{2}{*}{$\begin{array}{c}\mathrm{PA}_{\operatorname{morph}}\left(r_{\max }\right) \\
\left({ }^{\circ}\right) \\
(7)\end{array}$} & \multicolumn{2}{|c|}{ PA approaching } & \multicolumn{2}{|c|}{ PA receding } \\
\hline & & & & & & & $\begin{array}{l}\mathrm{PA}_{\text {kin }} \\
\left({ }^{\circ}\right) \\
(8) \\
\end{array}$ & $\begin{array}{c}\delta \mathrm{PA}_{\mathrm{kin}} \\
\left({ }^{\circ}\right) \\
(9)\end{array}$ & $\begin{array}{l}\mathrm{PA}_{\mathrm{kin}} \\
\left({ }^{\circ}\right) \\
(10) \\
\end{array}$ & $\begin{array}{c}\delta \mathrm{PA}_{\mathrm{kin}} \\
\left({ }^{\circ}\right) \\
(11)\end{array}$ \\
\hline 790 & $1.8 \pm 0.3$ & $0.9 \pm 0.3$ & $4717 \pm 6$ & 12 & 5 & $143.6 \pm 1.3$ & $145.5 \pm 3.7$ & $11.1 \pm 1.9$ & $160.7 \pm 5.9$ & $12.7 \pm 3.3$ \\
\hline 791 & $-0.5 \pm 0.2$ & $-0.3 \pm 0.2$ & $3276 \pm 4$ & 30 & $\ldots$ & $152.3 \pm 0.3$ & $154.1 \pm 1.1$ & $3.8 \pm 0.7$ & $148.9 \pm 1.0$ & $3.2 \pm 0.4$ \\
\hline 804 & $-3.1 \pm 0.4$ & $-2.4 \pm 0.5$ & $3486 \pm 7$ & 15 & $\ldots$ & $126.8 \pm 0.5$ & $124.7 \pm 4.7$ & $25.2 \pm 6.7$ & $109.5 \pm 15.9$ & $71.6 \pm 12.6$ \\
\hline 810 & $0.7 \pm 0.1$ & $1.1 \pm 0.1$ & $4093 \pm 1$ & 25 & $\ldots$ & $15.3 \pm 0.5$ & $15.4 \pm 0.7$ & $4.1 \pm 0.8$ & $15.1 \pm 0.6$ & $5.7 \pm 0.8$ \\
\hline 813 & $-0.6 \pm 0.1$ & $0.0 \pm 0.2$ & $3818 \pm 1$ & 28 & 7 & $113.1 \pm 8.8$ & $101.9 \pm 2.0$ & $15.8 \pm 2.7$ & $90.5 \pm 3.0$ & $13.8 \pm 3.8$ \\
\hline 823 & $-1.0 \pm 0.2$ & $1.9 \pm 0.3$ & $2825 \pm 2$ & 25 & 7 & $159.2 \pm 0.6$ & $149.6 \pm 2.1$ & $8.3 \pm 1.4$ & $158.6 \pm 2.1$ & $10.7 \pm 2.0$ \\
\hline 824 & $-0.4 \pm 0.1$ & $-0.6 \pm 0.2$ & $4732 \pm 3$ & 27 & $\ldots$ & $159.8 \pm 0.2$ & $159.3 \pm 1.0$ & $5.4 \pm 1.2$ & $161.7 \pm 1.2$ & $5.5 \pm 1.1$ \\
\hline 826 & $\ldots$ & $\ldots$ & $5014 \pm 2$ & 25 & $\ldots$ & $127.9 \pm 0.3$ & $123.9 \pm 1.8$ & $12.4 \pm 2.2$ & $129.9 \pm 2.2$ & $17.9 \pm 3.3$ \\
\hline 831 & $0.2 \pm 0.1$ & $0.5 \pm 0.2$ & $4968 \pm 4$ & 15 & $\ldots$ & $123.4 \pm 0.6$ & $124.2 \pm 1.5$ & $6.6 \pm 1.2$ & $127.1 \pm 1.2$ & $5.3 \pm 0.9$ \\
\hline 834 & $-0.4 \pm 0.5$ & $1.3 \pm 0.5$ & $8764 \pm 14$ & 15 & $\ldots$ & $114.2 \pm 0.5$ & $101.9 \pm 3.7$ & $11.8 \pm 4.3$ & $106.8 \pm 3.7$ & $7.5 \pm 2.1$ \\
\hline 836 & $1.1 \pm 0.2$ & $1.9 \pm 0.2$ & $2407 \pm 0$ & 29 & $\ldots$ & $148.9 \pm 0.9$ & $120.9 \pm 1.7$ & $10.9 \pm 1.9$ & $135.6 \pm 2.2$ & $17.5 \pm 2.6$ \\
\hline 842 & $0.0 \pm 0.3$ & $-0.2 \pm 0.3$ & $2955 \pm 6$ & 30 & 7 & $54.7 \pm 0.4$ & $56.8 \pm 2.4$ & $9.6 \pm 1.5$ & $63.2 \pm 2.6$ & $15.6 \pm 1.8$ \\
\hline 853 & $0.8 \pm 0.2$ & $-1.0 \pm 0.2$ & $3809 \pm 3$ & 30 & 10 & $53.0 \pm 5.0$ & $50.3 \pm 0.9$ & $2.9 \pm 0.3$ & $47.3 \pm 1.2$ & $3.3 \pm 0.4$ \\
\hline 854 & $0.9 \pm 0.4$ & $0.2 \pm 0.2$ & $8654 \pm 16$ & 20 & $\ldots$ & $90.9 \pm 0.4$ & $87.4 \pm 2.3$ & $10.1 \pm 2.8$ & $86.2 \pm 3.1$ & $9.7 \pm 2.9$ \\
\hline 856 & $0.7 \pm 0.1$ & $2.1 \pm 0.2$ & $4700 \pm 3$ & 22 & 5 & $91.6 \pm 0.7$ & $94.3 \pm 2.6$ & $10.4 \pm 2.5$ & $91.8 \pm 2.6$ & $6.9 \pm 1.8$ \\
\hline 857 & $-2.0 \pm 0.6$ & $-1.5 \pm 0.4$ & $8539 \pm 8$ & 15 & $\ldots$ & $36.1 \pm 0.4$ & $60.5 \pm 3.7$ & $12.6 \pm 5.6$ & $38.2 \pm 4.3$ & $11.7 \pm 3.1$ \\
\hline 862 & $-1.3 \pm 0.2$ & $1.0 \pm 0.2$ & $6720 \pm 4$ & 25 & $\ldots$ & $32.1 \pm 0.4$ & $27.8 \pm 1.2$ & $4.9 \pm 1.0$ & $37.8 \pm 1.7$ & $7.3 \pm 0.7$ \\
\hline 869 & $1.3 \pm 0.6$ & $3.0 \pm 0.4$ & $6139 \pm 18$ & 22 & 7 & $121.8 \pm 1.8$ & $125.9 \pm 3.0$ & $13.4 \pm 3.7$ & $133.0 \pm 2.7$ & $12.3 \pm 2.5$ \\
\hline 872 & $2.0 \pm 0.7$ & $1.1 \pm 0.6$ & $3813 \pm 22$ & 20 & 7 & $85.4 \pm 3.0$ & $64.3 \pm 5.3$ & $36.8 \pm 3.2$ & $45.3 \pm 6.7$ & $30.3 \pm 5.8$ \\
\hline 876 & $0.1 \pm 0.3$ & $-1.2 \pm 0.2$ & $5743 \pm 4$ & 20 & 5 & $106.9 \pm 1.3$ & $98.1 \pm 2.4$ & $8.8 \pm 1.7$ & $96.7 \pm 3.2$ & $14.2 \pm 3.8$ \\
\hline 886 & $-0.3 \pm 0.1$ & $0.1 \pm 0.2$ & $4455 \pm 5$ & 27 & 7 & $12.8 \pm 0.3$ & $10.3 \pm 1.2$ & $6.0 \pm 1.4$ & $17.2 \pm 1.1$ & $7.0 \pm 1.2$ \\
\hline 887 & $0.8 \pm 0.1$ & $0.9 \pm 0.1$ & $7056 \pm 3$ & 25 & 5 & $25.2 \pm 1.4$ & $15.3 \pm 1.8$ & $8.6 \pm 1.2$ & $15.0 \pm 1.6$ & $7.3 \pm 1.5$ \\
\hline 889 & $-0.5 \pm 0.0$ & $0.5 \pm 0.1$ & $4883 \pm 0$ & 20 & 10 & $64.4 \pm 0.7$ & $61.7 \pm 1.2$ & $6.3 \pm 0.9$ & $70.7 \pm 1.2$ & $5.4 \pm 1.2$ \\
\hline 890 & $2.1 \pm 0.4$ & $-1.2 \pm 0.4$ & $6533 \pm 5$ & 15 & $\ldots$ & $142.6 \pm 0.5$ & $144.5 \pm 3.0$ & $13.9 \pm 4.0$ & $167.8 \pm 4.2$ & $9.7 \pm 3.8$ \\
\hline 896 & $0.9 \pm 0.2$ & $0.0 \pm 0.3$ & $7461 \pm 6$ & 18 & $\ldots$ & $19.6 \pm 0.5$ & $18.2 \pm 2.7$ & $7.7 \pm 1.4$ & $28.1 \pm 1.8$ & $6.8 \pm 1.7$ \\
\hline 904 & $-0.6 \pm 0.1$ & $1.5 \pm 0.1$ & $4892 \pm 0$ & 30 & 6 & $148.2 \pm 1.1$ & $145.1 \pm 1.5$ & $8.4 \pm 1.1$ & $147.2 \pm 0.7$ & $5.9 \pm 1.1$ \\
\hline 924 & $-0.2 \pm 0.4$ & $-0.3 \pm 0.4$ & $2525 \pm 12$ & 22 & 7 & $24.6 \pm 0.8$ & $49.3 \pm 3.9$ & $13.4 \pm 2.9$ & $52.0 \pm 2.7$ & $13.8 \pm 3.7$ \\
\hline 929 & $-2.0 \pm 0.3$ & $1.4 \pm 0.3$ & $8103 \pm 6$ & 20 & $\ldots$ & $43.4 \pm 0.8$ & $52.4 \pm 1.3$ & $7.1 \pm 1.5$ & $50.7 \pm 1.9$ & $7.6 \pm 1.8$ \\
\hline 938 & $0.6 \pm 0.2$ & $0.7 \pm 0.2$ & $5913 \pm 3$ & 15 & 7 & $51.1 \pm 5.9$ & $64.6 \pm 3.9$ & $14.6 \pm 3.4$ & $68.4 \pm 5.2$ & $12.3 \pm 3.4$ \\
\hline
\end{tabular}


J. K. Barrera-Ballesteros et al.: Kinematic alignment of non-interacting CALIFA galaxies

Table B.4. Kinematic orientation measured at different radius for a sample of low inclined galaxies included in our sample of non-interacting galaxies.

\begin{tabular}{|c|c|c|c|c|c|c|c|c|c|c|c|c|}
\hline \multirow[t]{3}{*}{ id } & \multirow[b]{3}{*}{$\begin{array}{c}r_{\text {in }}^{\text {stars }} \\
(\operatorname{arcsec}) \\
(2)\end{array}$} & \multirow[b]{3}{*}{$\begin{array}{c}r_{\text {out }}^{\text {stars }} \\
(\operatorname{arcsec}) \\
(3)\end{array}$} & \multirow[b]{3}{*}{$\begin{array}{c}r_{\text {in }}^{\text {gas }} \\
(\operatorname{arcsec}) \\
(4)\end{array}$} & \multirow[b]{3}{*}{$\begin{array}{c}r_{\text {out }}^{\text {gas }} \\
(\operatorname{arcsec}) \\
(5)\end{array}$} & \multicolumn{4}{|c|}{ Stars } & \multicolumn{4}{|c|}{ Ionized gas } \\
\hline & & & & & \multicolumn{2}{|c|}{$r_{\text {in }}$} & \multicolumn{2}{|c|}{$r_{\text {out }}$} & \multicolumn{2}{|c|}{$r_{\text {in }}$} & \multicolumn{2}{|c|}{$r_{\text {out }}$} \\
\hline & & & & & $\begin{array}{c}\mathrm{PA}_{\mathrm{app}} \\
\text { (degrees) } \\
(6)\end{array}$ & $\begin{array}{c}\mathrm{PA}_{\text {rec }} \\
\text { (degrees) } \\
(7)\end{array}$ & $\begin{array}{c}\mathrm{PA}_{\mathrm{app}} \\
\text { (degrees) } \\
(8)\end{array}$ & $\begin{array}{c}\mathrm{PA}_{\text {rec }} \\
\text { (degrees) } \\
(9)\end{array}$ & $\begin{array}{c}\mathrm{PA}_{\mathrm{app}} \\
\text { (degrees) } \\
(10)\end{array}$ & $\begin{array}{c}\mathrm{PA}_{\text {rec }} \\
\text { (degrees) } \\
(11)\end{array}$ & $\begin{array}{c}\mathrm{PA}_{\mathrm{app}} \\
\text { (degrees) } \\
(12)\end{array}$ & $\begin{array}{c}\mathrm{PA}_{\text {rec }} \\
\text { (degrees) } \\
(13)\end{array}$ \\
\hline 8 & 10 & 20 & 14 & 28 & 114 & 117 & 114 & 112 & 114 & 114 & 114 & 117 \\
\hline 10 & 12 & 25 & 12 & 27 & 27 & 15 & 12 & 12 & 18 & 13 & 15 & 17 \\
\hline 23 & 15 & 25 & 27 & 30 & 81 & 84 & 103 & 89 & 77 & 102 & 76 & 76 \\
\hline 25 & 22 & 30 & 22 & 30 & 170 & 166 & 155 & 171 & 170 & 166 & 168 & 155 \\
\hline 28 & 14 & 17 & 14 & 28 & 52 & 42 & 72 & 52 & 55 & 48 & 49 & 51 \\
\hline 30 & 7 & 20 & 7 & 30 & 183 & 162 & 189 & 168 & 12 & 162 & 6 & 91 \\
\hline 31 & 15 & 30 & 16 & 32 & 57 & 74 & 72 & 64 & 67 & 72 & 65 & 73 \\
\hline 33 & 10 & 20 & 16 & 32 & 93 & 90 & 88 & 95 & 91 & 86 & 90 & 95 \\
\hline 43 & 8 & 17 & 8 & 17 & 22 & 29 & 9 & 20 & 7 & 19 & 99 & 10 \\
\hline 108 & 10 & 20 & 10 & 27 & 86 & 90 & 96 & 107 & 94 & 95 & 100 & 97 \\
\hline 147 & 10 & 20 & 10 & 30 & 117 & 116 & 112 & 125 & 117 & 115 & 117 & 115 \\
\hline 149 & 12 & 25 & 12 & 28 & 10 & 10 & 6 & 4 & 5 & 18 & 5 & 12 \\
\hline 275 & 13 & 27 & 16 & 32 & 78 & 76 & 79 & 94 & 83 & 80 & 82 & 86 \\
\hline 277 & 12 & 25 & 16 & 30 & 15 & 20 & 23 & 13 & 49 & 46 & 12 & 21 \\
\hline 414 & 14 & 25 & 14 & 30 & 137 & 136 & 147 & 144 & 133 & 137 & 146 & 152 \\
\hline 436 & 14 & 25 & 23 & 25 & 164 & 181 & 178 & 175 & 172 & 173 & 178 & 178 \\
\hline 489 & 13 & 27 & 16 & 32 & 102 & 110 & 95 & 102 & 98 & 106 & 105 & 99 \\
\hline 515 & 15 & 25 & 16 & 32 & 162 & 168 & 163 & 172 & 164 & 162 & 166 & 169 \\
\hline 518 & 17 & 30 & 17 & 30 & 105 & 93 & 90 & 98 & 104 & 97 & 95 & 98 \\
\hline 580 & 11 & 23 & 13 & 27 & 35 & 37 & 47 & 33 & 35 & 31 & 42 & 35 \\
\hline 602 & 11 & 22 & 5 & 10 & 68 & 81 & 75 & 84 & 71 & 80 & 65 & 63 \\
\hline 624 & 21 & 25 & 21 & 30 & 90 & 82 & 105 & 100 & 74 & 70 & 92 & 89 \\
\hline 630 & 12 & 22 & 12 & 32 & 160 & 97 & 155 & 158 & 167 & 163 & 159 & 157 \\
\hline 684 & 15 & 27 & 20 & 30 & 119 & 111 & 121 & 105 & 114 & 114 & 107 & 108 \\
\hline 715 & 15 & 30 & 16 & 32 & 72 & 59 & 66 & 61 & 65 & 67 & 64 & 58 \\
\hline 743 & 12 & 25 & 16 & 32 & 80 & 84 & 94 & 78 & 81 & 84 & 81 & 83 \\
\hline 748 & 12 & 25 & 16 & 30 & 12 & 20 & 18 & 19 & 12 & 24 & 13 & 22 \\
\hline 753 & 12 & 25 & 16 & 32 & 54 & 57 & 55 & 54 & 53 & 57 & 62 & 56 \\
\hline 764 & 7 & 20 & 7 & 30 & 132 & 124 & 119 & 138 & 120 & 113 & 130 & 128 \\
\hline 768 & 7 & 15 & 14 & 28 & 38 & 25 & 42 & 42 & 35 & 53 & 38 & 47 \\
\hline 771 & 10 & 20 & 21 & 30 & 21 & 33 & 35 & 38 & 39 & 41 & 48 & 33 \\
\hline 777 & 15 & 30 & 18 & 35 & 36 & 38 & 32 & 38 & 38 & 34 & 36 & 34 \\
\hline 790 & 6 & 12 & 10 & 20 & 137 & 143 & 144 & 146 & 144 & 162 & 149 & 155 \\
\hline 791 & 15 & 30 & 15 & 30 & 151 & 154 & 149 & 154 & 156 & 149 & 153 & 149 \\
\hline 813 & 16 & 28 & 16 & 35 & 106 & 88 & 93 & 88 & 101 & 83 & 99 & 95 \\
\hline 823 & 12 & 25 & 16 & 32 & 156 & 165 & 160 & 161 & 149 & 162 & 152 & 154 \\
\hline 836 & 14 & 29 & 14 & 29 & 144 & 135 & 131 & 129 & 127 & 138 & 119 & 129 \\
\hline 842 & 18 & 30 & 15 & 30 & 90 & 73 & 57 & 60 & 58 & 70 & 55 & 52 \\
\hline 853 & 15 & 30 & 15 & 30 & 53 & 46 & 56 & 50 & 49 & 45 & 50 & 48 \\
\hline 856 & 7 & 22 & 7 & 30 & 84 & 98 & 95 & 89 & 76 & 88 & 94 & 93 \\
\hline 869 & 16 & 22 & 16 & 30 & 130 & 142 & 116 & 132 & 134 & 136 & 124 & 133 \\
\hline 872 & 17 & 20 & 17 & 30 & 63 & 55 & 75 & 65 & 61 & 39 & 73 & 71 \\
\hline 876 & 10 & 20 & 15 & 25 & 94 & 79 & 96 & 110 & 102 & 89 & 92 & 101 \\
\hline 886 & 13 & 27 & 16 & 32 & 2 & 24 & 4 & 18 & 15 & 16 & 7 & 15 \\
\hline 887 & 10 & 25 & 10 & 35 & 10 & 10 & 15 & 24 & 11 & 15 & 14 & 13 \\
\hline 889 & 11 & 22 & 11 & 28 & 71 & 58 & 74 & 58 & 57 & 66 & 63 & 69 \\
\hline 904 & 11 & 30 & 11 & 30 & 143 & 138 & 152 & 146 & 142 & 143 & 145 & 148 \\
\hline 924 & 11 & 22 & 16 & 32 & 39 & 48 & 33 & 51 & 43 & 53 & 46 & 48 \\
\hline 938 & 11 & 20 & 11 & 30 & 78 & 72 & 67 & 74 & 64 & 69 & 66 & 66 \\
\hline
\end{tabular}

Notes. (1) CALIFA identifier. (2) and (3) internal and external radii used to measured the kinematic PA in the stellar component (see Sect. 4.3 for details). (4) and (5) internal and external radii used to measured the kinematic PA in the ionized gas component. (6) and (7) internal approaching and receding kinematic position angles for the stellar component (see Sect. 4.3 for details). (8) and (9) external approaching and receding kinematic position angles for the stellar component. (10) and (11) internal approaching and receding kinematic position angles for the ionized gas. (12) and (13) external approaching and receding kinematic position angles for the ionized gas. 


\section{Appendix C: Stellar and lonised velocity fields}

In this appendix we present the velocity field of the 80 noninteracting galaxies. Each row present one galaxy similar to the one in Fig. 1, from left to right: SDSS $r$-band image of the galaxy. The white solid line represents the photometric PA measured at the same galactocentric distance as the kinematic PA ( $\mathrm{PA}_{\text {morph }}$, see Sect. 3). The dashed line represents the photometric PA measured at the outermost isophote of the image ( $\mathrm{PA}_{\text {morph }}^{\text {out }}$, see Sect. 4.3). The next two panels show the stellar and ionised gas velocity maps, respectively. Green points highlight the locations where the maximum velocity is located at a given radius, determined from the position-velocity diagram.
Black lines for each kinematic side represent the average kinematic PA $\left(\mathrm{PA}_{\mathrm{kin}}\right)$, while white thin lines along the zero-velocity curve show the average minor kinematic PA. The next panel shows the distance from the galactic centre versus the maximum for the stellar (blue) and ionised gas (red) components. The curve along $0 \mathrm{~km} \mathrm{~s}^{-1}$ represents the velocities along the zero-velocity curve. Uncertainty in velocity are determined from Monte Carlo simulations. The last panel shows the dependence of the different $\mathrm{PA}$ with respect to the radius. $\mathrm{PA}_{\text {morph }}, \mathrm{PA}_{\text {kin }}^{\text {stellar }}$, and $\mathrm{PA}_{\text {kin }}^{\mathrm{gas}}$ are represented by open circles, filled blue diamonds, and filled red squares, respectively. For barred galaxies, we highlight the length of the bar and its orientation with dashed lines. 
J. K. Barrera-Ballesteros et al.: Kinematic alignment of non-interacting CALIFA galaxies
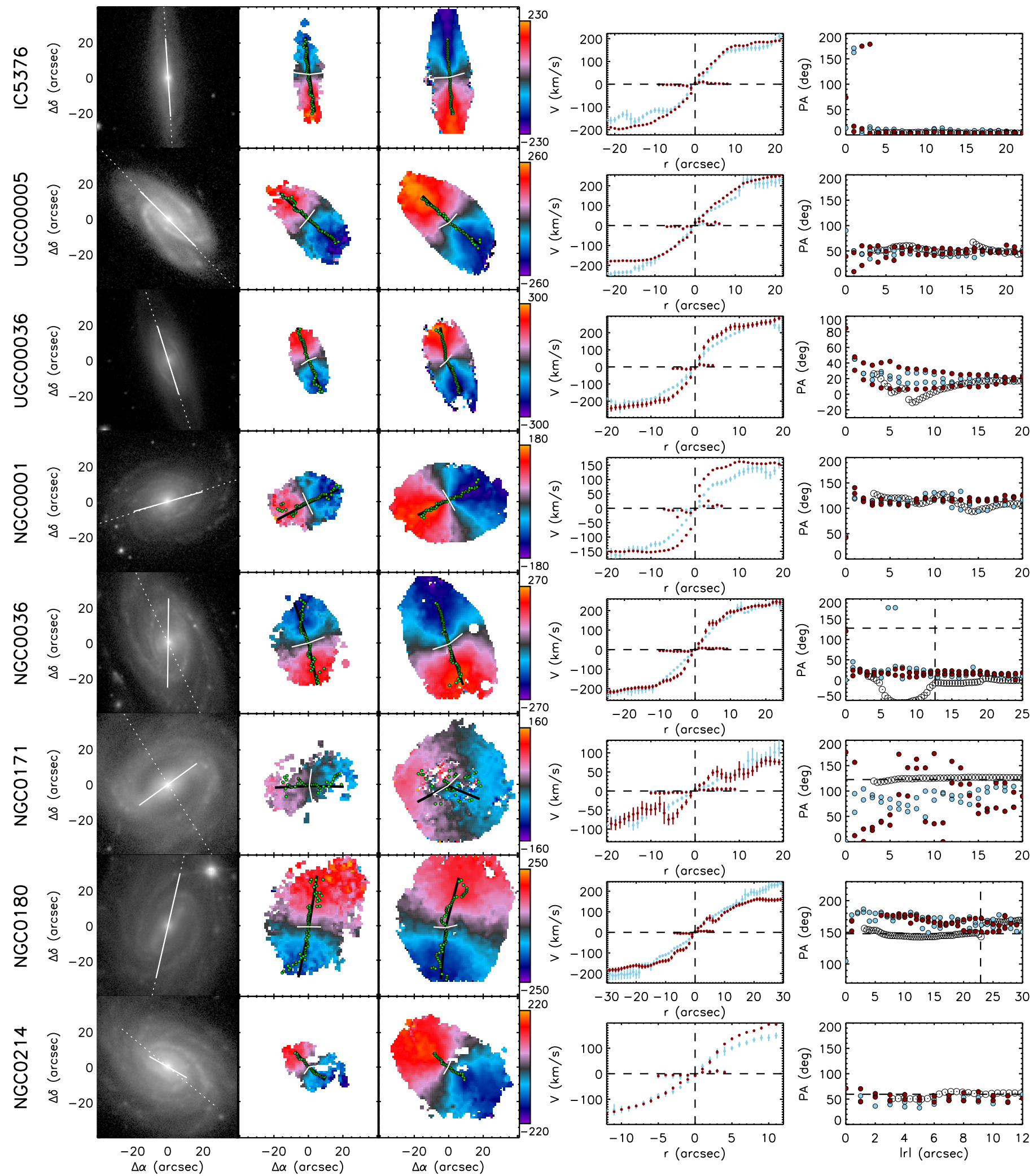

Fig. C.1. Stellar and ionised gas velocity fields for the sample of galaxies used in this study. 
A\&A 568, A70 (2014)
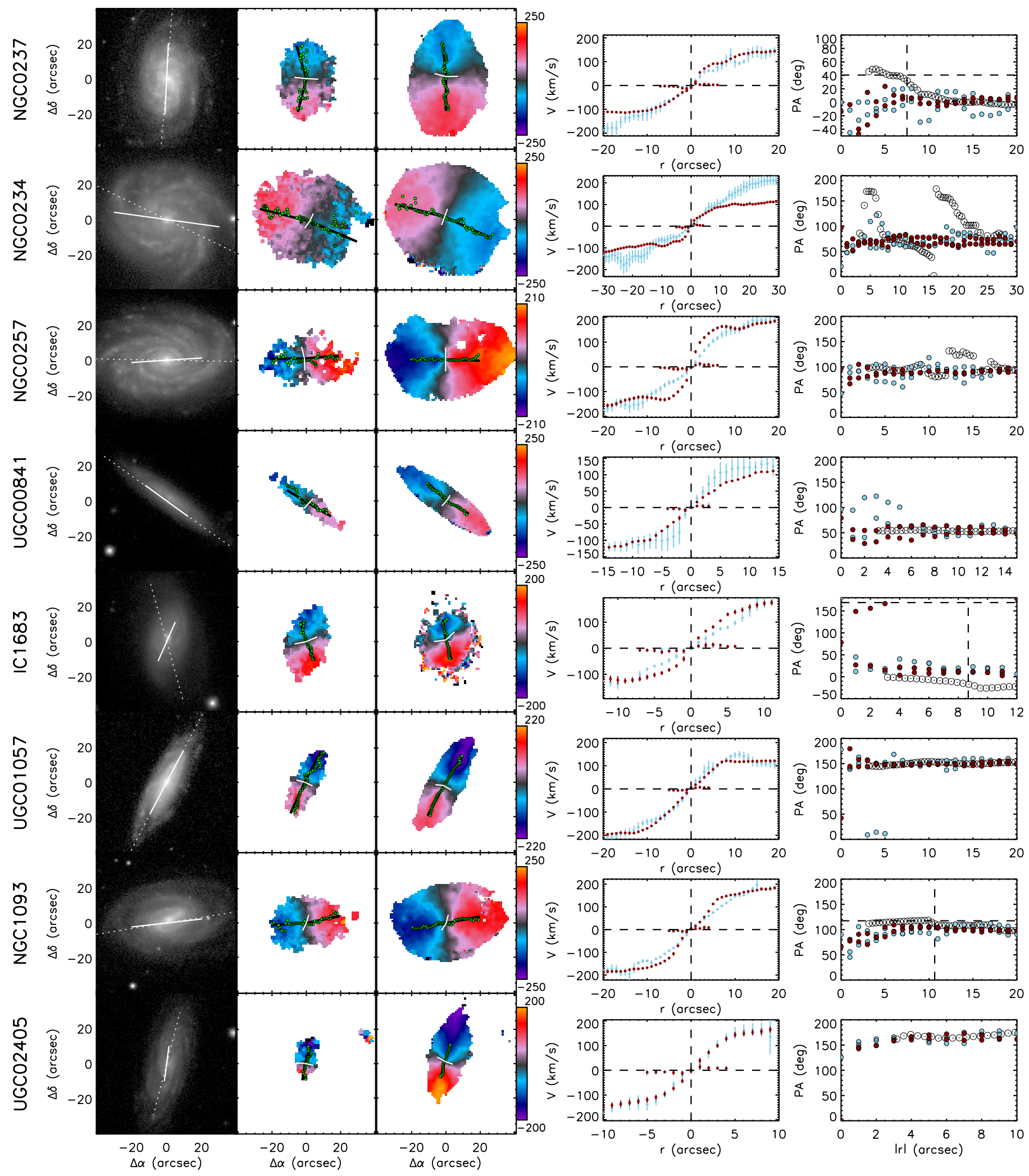

Fig. C.1. continued. 
J. K. Barrera-Ballesteros et al.: Kinematic alignment of non-interacting CALIFA galaxies
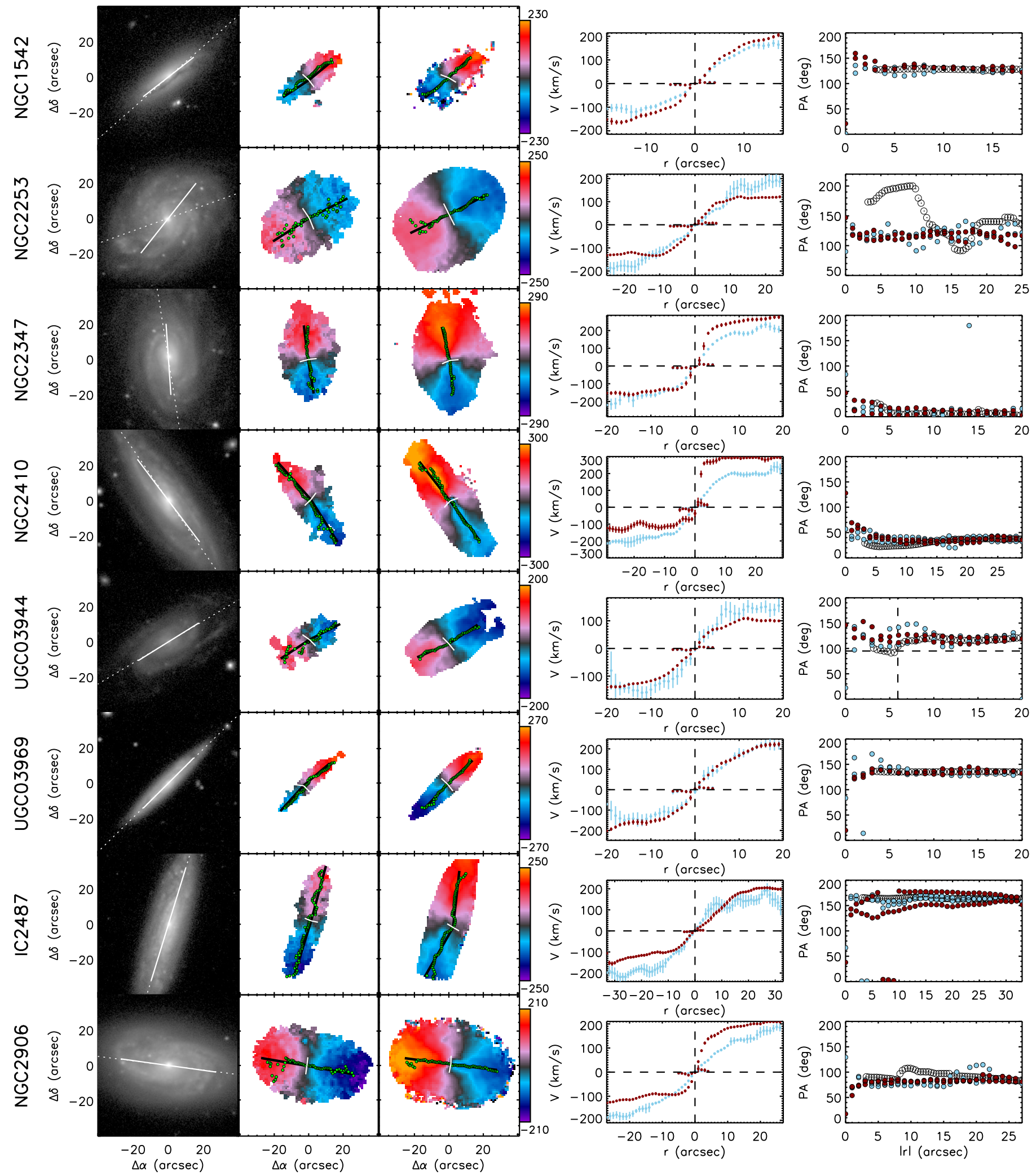

Fig. C.1. continued. 


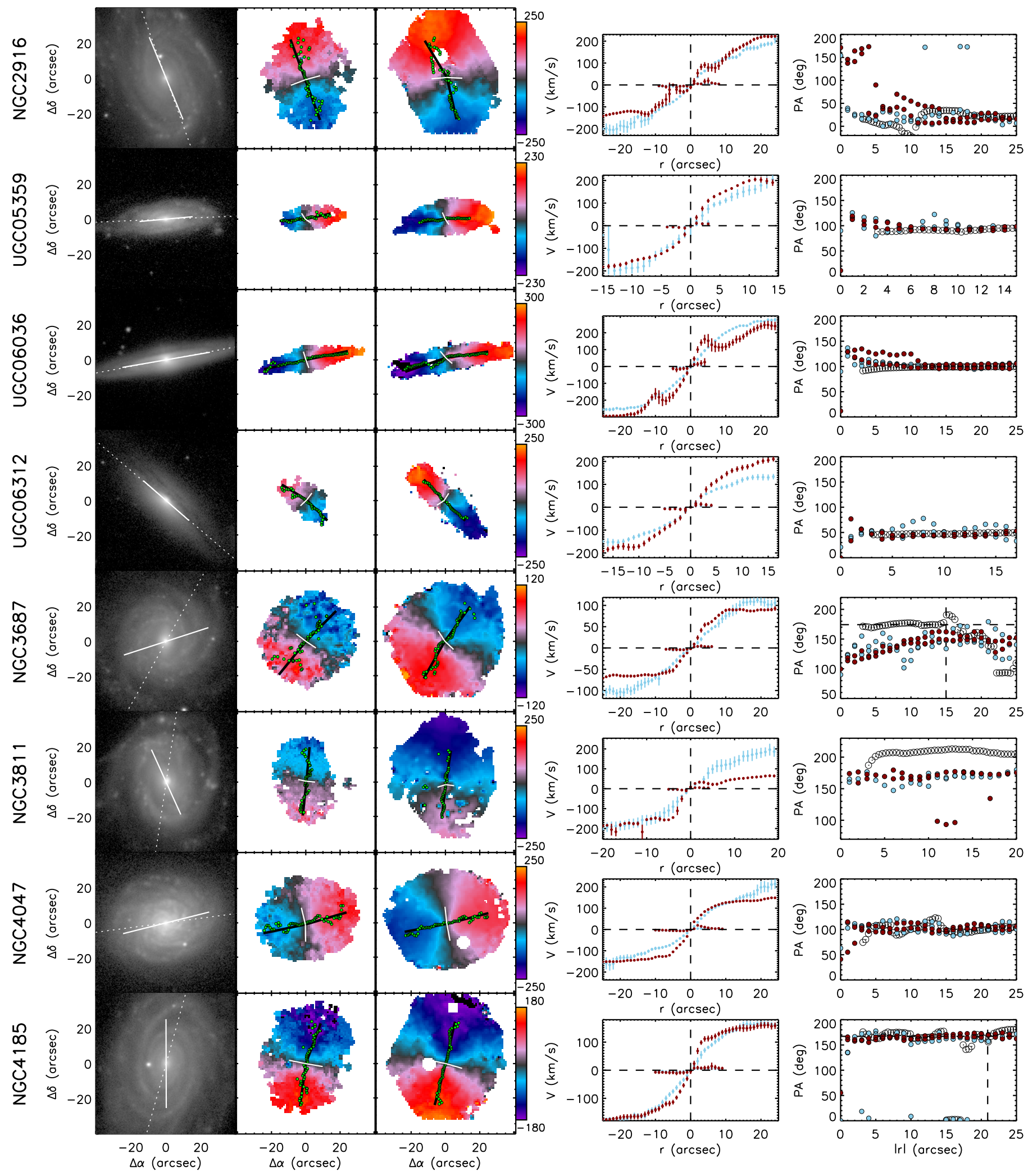

Fig. C.1. continued. 
J. K. Barrera-Ballesteros et al.: Kinematic alignment of non-interacting CALIFA galaxies
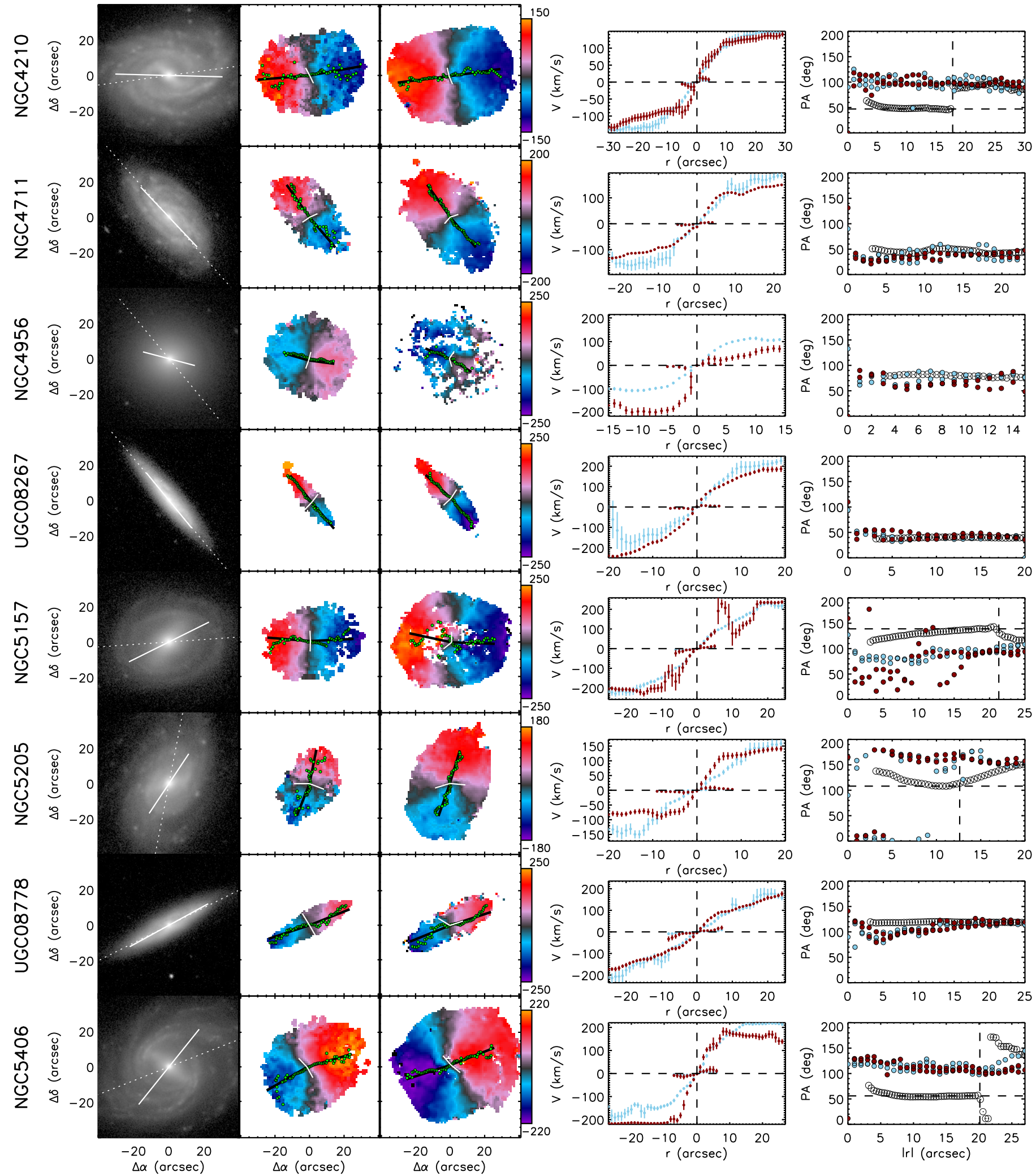

Fig. C.1. continued. 


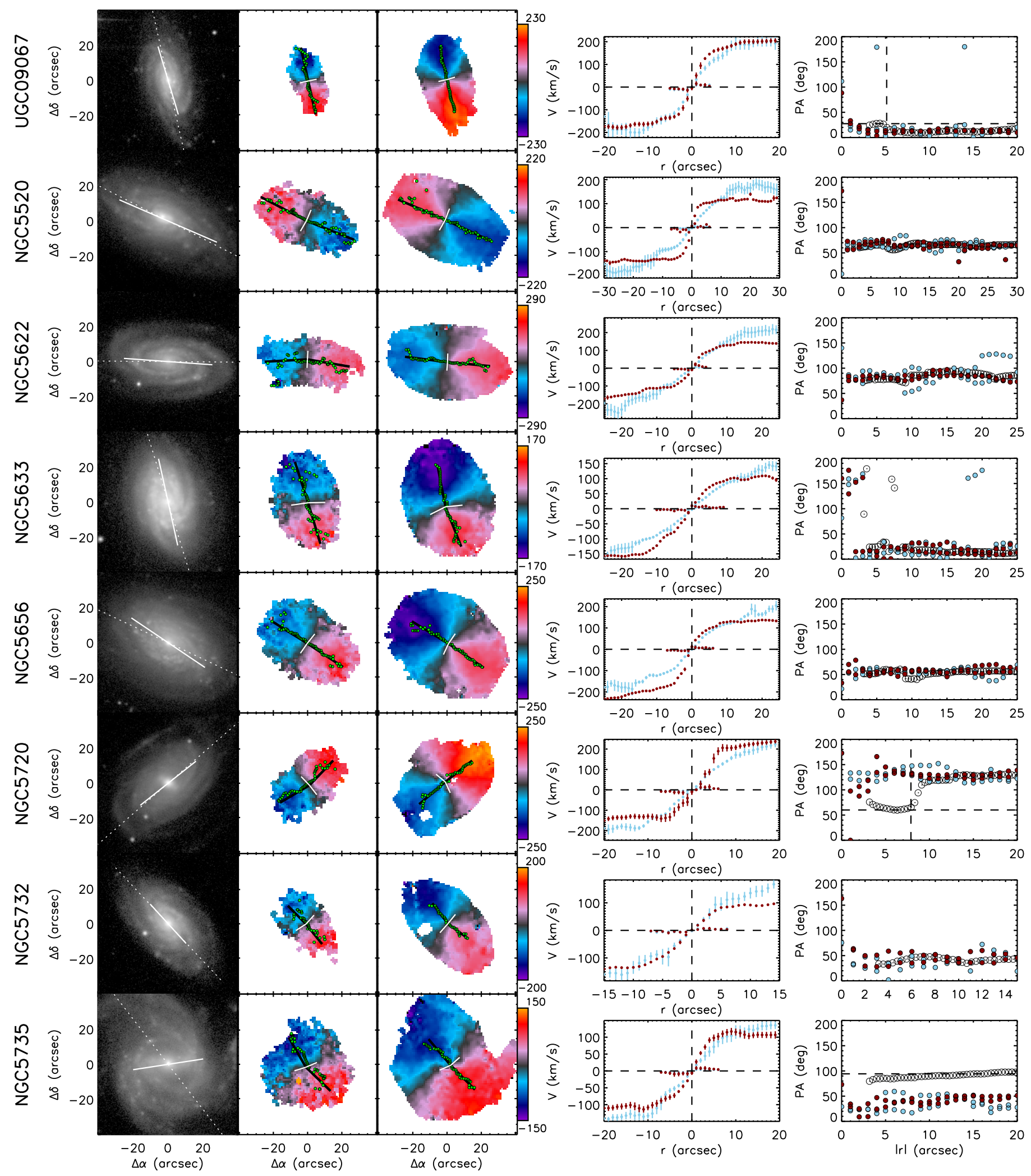

Fig. C.1. continued. 
J. K. Barrera-Ballesteros et al.: Kinematic alignment of non-interacting CALIFA galaxies
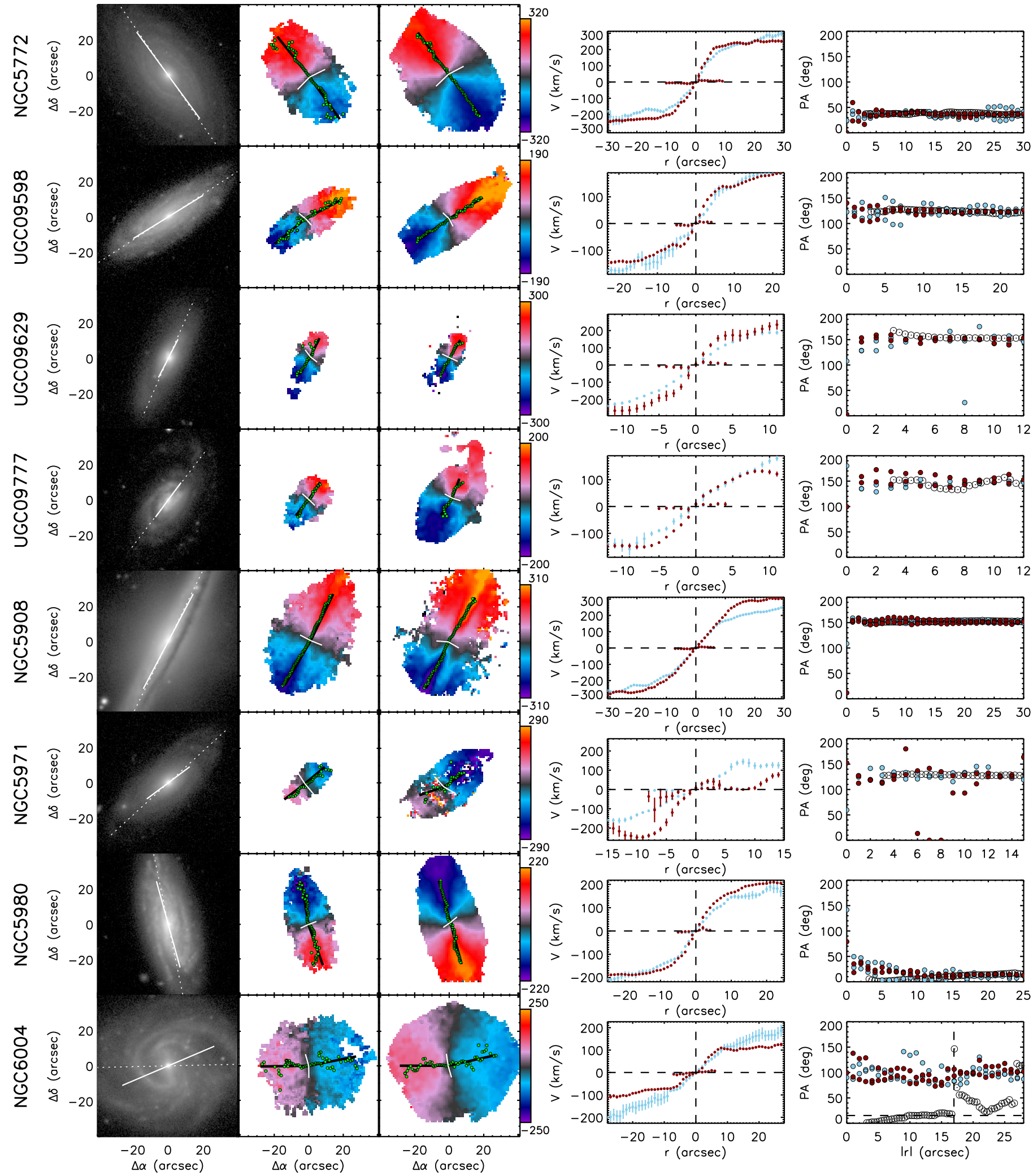

Fig. C.1. continued. 


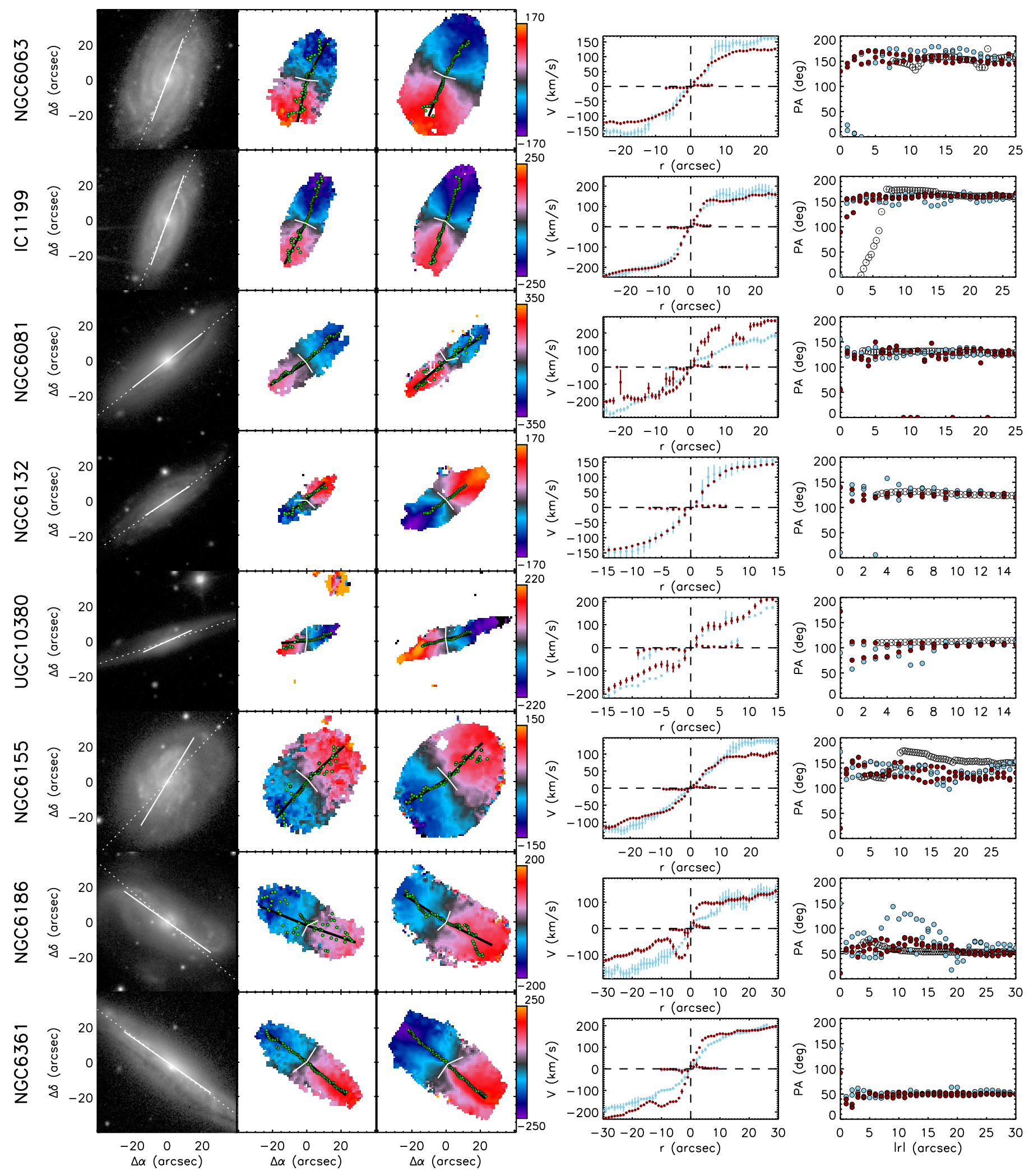

Fig. C.1. continued. 
J. K. Barrera-Ballesteros et al.: Kinematic alignment of non-interacting CALIFA galaxies
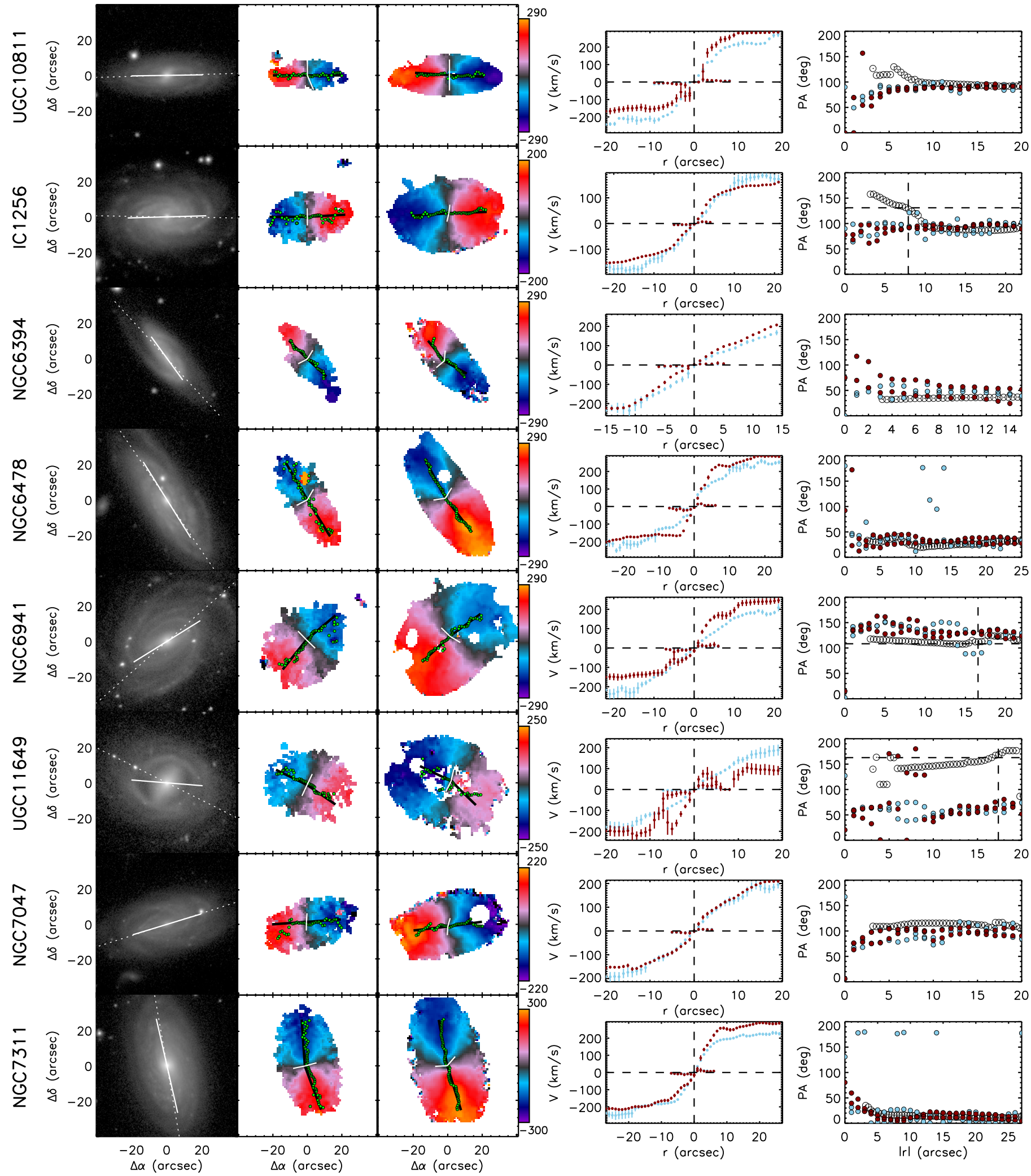

Fig. C.1. continued. 


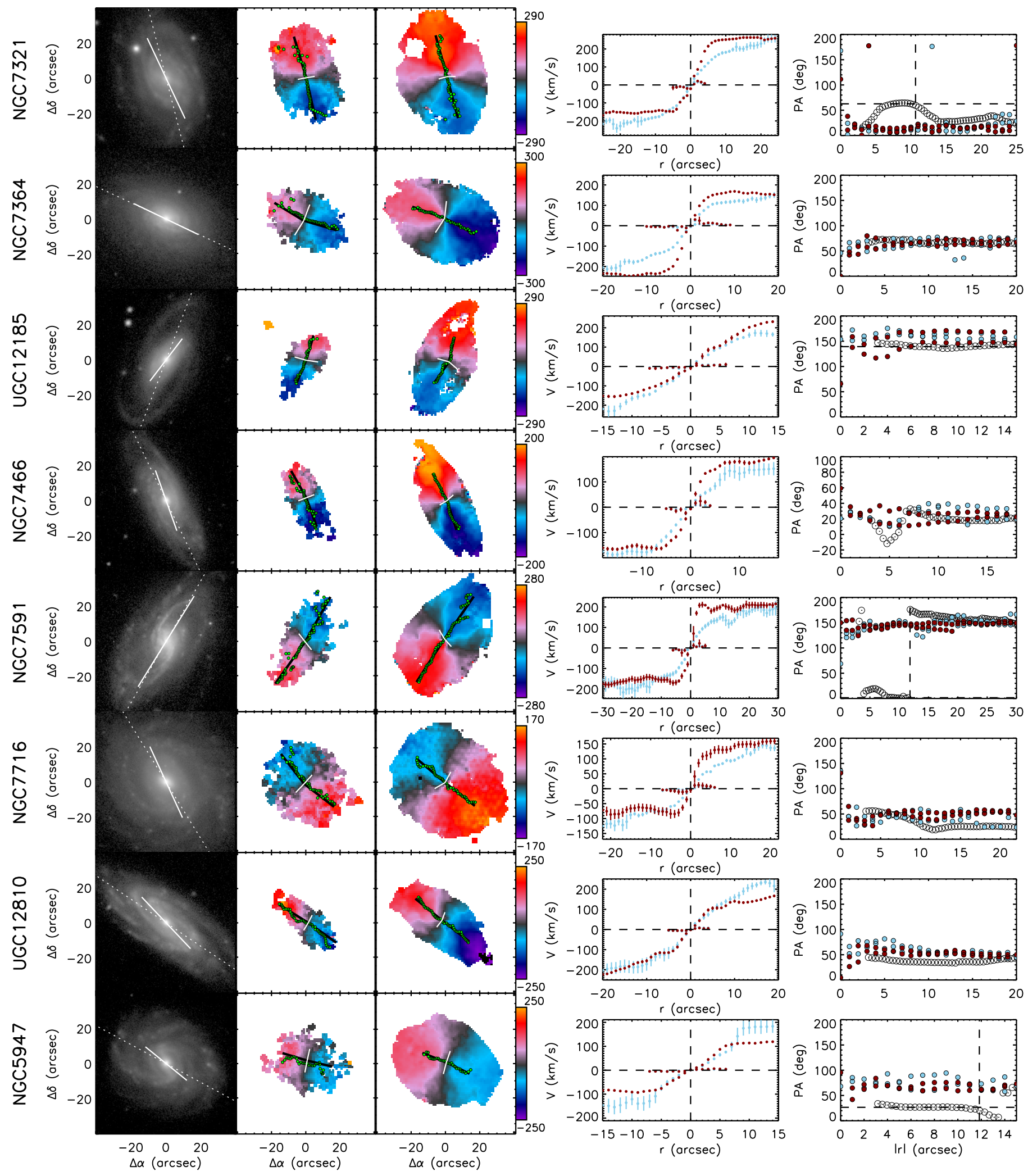

Fig. C.1. continued. 